\title{
Rotating Trapped Bose-Einstein Condensates
}

\author{
Alexander L. Fetter \\ Geballe Laboratory for Advanced Materials and Departments of Physics and Applied Physics, Stanford University, Stanford, \\ CA 94305-4045, USA
}

(Dated: May 28, 2018)

\begin{abstract}
After reviewing the ideal Bose-Einstein gas in a box and in a harmonic trap, I discuss the effect of interactions on the formation of a Bose-Einstein condensate (BEC), along with the dynamics of small-amplitude perturbations (the Bogoliubov equations). When the condensate rotates with angular velocity $\Omega$, one or several vortices nucleate, with many observable consequences. With more rapid rotation, the vortices form a dense triangular array, and the collective behavior of these vortices has additional experimental implications. For $\Omega$ near the radial trap frequency $\omega_{\perp}$, the lowest-Landau-level approximation becomes applicable, providing a simple picture of such rapidly rotating condensates. Eventually, as $\Omega \rightarrow \omega_{\perp}$, the rotating dilute gas is expected to undergo a quantum phase transition from a superfluid to various highly correlated (nonsuperfluid) states analogous to those familiar from the fractional quantum Hall effect for electrons in a strong perpendicular magnetic field.
\end{abstract}

PACS numbers: $03.75 . \mathrm{Hh}, 05.30 . J p, 67.40 . \mathrm{Db}$

\section{Contents}

\section{Introduction}

II. Physics of Bose-Einstein Condensates in Dilute Trapped Gases

A. Ideal Bose gas

1. Ideal BEC in a box with periodic boundary conditions

2. Ideal BEC in a harmonic trap

B. Inclusion of interparticle interactions

C. Bogoliubov equations

III. Physics of One Vortex (a Few Vortices) in a Trap 8

A. One vortex in unbounded condensate

B. One vortex in a trapped condensate

1. Gross-Pitaevskii energy in a trap

2. Dynamical motion of a trapped vortex

C. Small vortex arrays in a trap

D. Experimental and theoretical studies in a trap

1. Vortex behavior in two-component BEC mixtures (JILA)

2. Vortex behavior in one-component BECs (ENS and subsequently others)

3. Normal modes of an axisymmetric condensate with one central vortex

4. Bent vortex configurations

5. Kelvin (axial) waves on a vortex

6. Other ways to create vortices

E. Laser trapping and optical lattices

F. Berezinskii-Kosterlitz-Thouless transition in dilute BECs

IV. Vortex Arrays in Mean-Field Thomas-Fermi (TF) Regime

A. Physics of BEC in axisymmetric harmonic traps for rapid rotation

B. Experimental and theoretical studies of vortex lattices in the $\mathrm{TF}$ regime

*Electronic address: fetter@stanford.edu
1. Collective modes of rotating condensates

2. Uniformity of the vortex array

3. Vortex core size for large rotation speeds

4. Tkachenko oscillations of the vortex lattice

5. Rotating two-component BECs

6. Rotating gas of paired fermions in tightly bound (BEC) limit

C. Effect of additional quartic confinement

V. Vortex Arrays in Mean-Field Lowest-Landau-Level (LLL) Regime

A. Physics of lowest-Landau-level (LLL) one-body states 32

B. Direct treatment of LLL states

C. LLL condensate wave functions

1. Unrestricted minimization

2. Inclusion of the vortices

3. Rapidly rotating anisotropic traps

VI. Quantum Phase Transition to Highly Correlated States

VII. Outlook

A. Successes

B. Challenges

1. Rapid rotation of both Bose and Fermi gases in the quantum Hall regime at small filling fraction

2. Rotation of ultracold dilute gases with long-range dipole-dipole interactions

3. Rotating spinor condensates

Acknowledgments

References

24

\section{INTRODUCTION}

The remarkable creation of Bose-Einstein condensates (BECs) in cold dilute alkalai-metal gases (Anderson et al., 1995; Bradley et al., 1995; Davis et al., 1995) has produced a wholly new exciting field that continues to thrive. Typically, these systems have a macroscopic condensate wave function $\Psi(\boldsymbol{r}, t)$ that character- 
izes the static and dynamic behavior of the BEC [for a general background, see, for example, Dalfovo et al. (1999); Inguscio et al. (1999); Pethick and Smith (2002); Pitaevskii and Stringari (2003)]. In the usual limit of a dilute gas, $\Psi$ obeys a nonlinear Schrödinger equation known as the Gross-Pitaevskii equation (Gross, 1961; Pitaevskii, 1961).

Subsequent experiments formed rotating condensates, initially with a small number of vortices (Madison et al., 2000a; Matthews et al., 1999a), and then with large vortex arrays (Abo-Shaeer et al., 2001; Haljan et al., $2001 \mathrm{~b}$ ). As in the case of rotating superfluid ${ }^{4} \mathrm{He}$ (Donnelly, 1991), the vortices have quantized circulation, arising from the single-valued nature of the condensate wave function $\Psi$. Nevertheless, the new dilutegas BECs differ considerably from superfluid ${ }^{4} \mathrm{He}$ because the Gross-Pitaevskii equation here provides a remarkably detailed description of the physics, allowing a careful comparison of theory and experiment.

This article focuses on the physics of quantized vortices in ultracold dilute trapped quantum gases. I first review the dilute Bose-Einstein gas, both in a box where the density is uniform and in harmonic traps where the density is nonuniform (Sec. II). The presence of (repulsive) interactions have a dramatic effect, leading to (1) a collective mode with a linear long-wavelength dispersion relation and (2) superfluidity of macroscopic flow. Section III treats the behavior of one vortex (or a small number of vortices) in a rotating condensate. For more rapid rotations, the vortices form a regular array that has many analogies with rotating ${ }^{4} \mathrm{He}$ and with the flux-line lattice in type-II superconductors (Sec. IV). As the rotation rate $\Omega$ continues to increase and approaches the radial trapping frequency $\omega_{\perp}$, the effective radial trapping potential weakens, and condensate expands. In this limit, the system enters a new two-dimensional superfluid regime that has close analogies with the lowest-Landau-level description of an electron in a uniform magnetic field (Sec. V). Ultimately, in the limit $\Omega / \omega_{\perp} \gtrsim 0.999$, the rotating Bose gas is predicted to make a quantum phase transition to one of several highly correlated ground states that are not superfluid (Sec. VI). This regime involves a sequence of many-body states that are similar to those developed for the quantum Hall effect (a two-dimensional electron gas in a strong magnetic field).

Throughout this article, I follow the lead of Bloch et al. (2007), focusing on models and predictions with experimental basis or confirmation. As a result, I treat only briefly two areas that have been studied in some experimental detail as nonrotating systems: (1) dipole condensates that interact with long-range electric or magnetic dipole forces [see, for example, Griesmaier et al. (2005); Lahave et al. (2007) and references therein] (2) spinor condensates [see, for example, Sadler et al. (2006); Stenger et al. (1998); Vengalattore et al. (2007) and references therein]. In both cases, however, rotation and the corresponding vortex structures remain an unexplored experimental capability (see, however, Sec. VII.B for an introductory discussion).

\section{PHYSICS OF BOSE-EINSTEIN CONDENSATES IN DILUTE TRAPPED GASES}

The general topic of Bose-Einstein condensates (BEC) in dilute trapped gases has been the focus of several reviews and books (Dalfovo et al., 1999; Inguscio et al., 1999; Pethick and Smith, 2002; Pitaevskii and Stringari, 2003). The current article will emphasize those aspects most relevant to rotating trapped gases and the associated quantized vortices (Aftalion, 2006; Fetter and Svidzinskv, 2001; Kasamatsu and Tsubota, 2007; Parker et al., 2007). In particular, I will emphasize the regime of regular vortex arrays, both the "mean-field Thomas-Fermi" limit when the vortex cores remain small and the "lowest-Landau-level" limit when the cores are comparable to the intervortex spacing (Baym, 2005; $\mathrm{Ho}$, 2001).

To review the basic facts of Bose-Einstein condensation, let us consider an ideal (noninteracting) uniform three-dimensional gas with $N$ particles in a cubical box (volume $V=L^{3}$ ) with mean density $n=N / V$. If the gas has a temperature $T$, the mean momentum per particle is $p_{T} \approx \sqrt{M k_{B} T}$, ignoring numerical factors of order unity, where $M$ is the atomic mass and $k_{B}$ is Boltzmann's constant. The de Broglie relation then yields the thermal wavelength $\lambda_{T} \approx h / p_{T} \approx h / \sqrt{M k_{B} T}$. The other relevant length is the interparticle spacing $n^{-1 / 3}$. The short-wavelength limit $\lambda_{T} \ll n^{-1 / 3}$ holds when $\hbar \rightarrow 0$ or $T$ is large; it describes the classical regime when quantum interference and diffraction are negligible (the analog of ray optics). As $T$ falls, however, this inequality eventually fails when $\lambda_{T} \approx n^{-1 / 3}$, or, equivalently, when the "phase-space density" defined as $n \lambda_{T}^{3}$ becomes of order unity. This condition signals the onset of quantum degeneracy; whenever $n \lambda_{T}^{3} \gtrsim 1$, quantum mechanics plays a central role in the physics.

The preceding description relies on an atomic-physics perspective, but a condensed-matter view yields a similar picture. In an ideal gas, each atom is effectively confined to a volume $n^{-1}$ by its neighbors, leading to a zero-point energy $\epsilon_{\mathrm{zp}} \approx \hbar^{2} n^{2 / 3} / M$. At high $T$ or low density $\left(k_{B} T \gg \epsilon_{\mathrm{zp}}\right)$, the system behaves classically, but when $k_{B} T \approx \epsilon_{\mathrm{zp}}$ (either because of reduced temperature or increased density), quantum effects again become crucial. These two characterizations of the onset of quantum degeneracy are essentially equivalent.

For ideal bosons, the critical temperature $T_{c}$ for the onset of quantum degeneracy leads to Bose-Einstein condensation, whereas for ideal Fermi gases, the similar onset temperature is known as the Fermi temperature $T_{F}$. For liquid helium with $n \approx 10^{22} \mathrm{~cm}^{-3}$, the appropriate transition temperature is of order $1 \mathrm{~K}$ for both isotopes $\left({ }^{4} \mathrm{He}\right.$ is a boson and ${ }^{3} \mathrm{He}$ is a fermion). A typical bosonic dilute alkalai-metal gas ( such as ${ }^{7} \mathrm{Li},{ }^{23} \mathrm{Na}$, and ${ }^{87} \mathrm{Rb}$ ) has a much lower $T_{c}$ of order $100-1000 \mathrm{nK}$, because 
of the larger atomic mass and reduced number density ( $n \approx 10^{13} \mathrm{~cm}^{-3}$ is a typical value for dilute cold gases). This article will focus on such bosonic systems.

The presence of Bose-Einstein condensation means that a single quantum state has macroscopic occupation. This particular quantum state acts like a particle reservoir that can absorb or emit excited particles with negligible change in its own properties. Thus it is natural to use the grand canonical ensemble in which the system of interest is assumed to be in equilibrium with a reservoir at temperature $T$ and chemical potential $\mu$ that determine the mean total energy and mean total number of particles.

\section{A. Ideal Bose gas}

Consider an ideal Bose gas in an external trap potential $V_{\mathrm{tr}}$, with a complete set of quantum-mechanical singleparticle energies $\epsilon_{j}$, and assume that the system is in equilibrium at temperature $T$ and chemical potential $\mu$. In the grand canonical ensemble, the mean occupation of the $j$ th state is

$$
n_{j}=\frac{1}{\exp \left[\beta\left(\epsilon_{j}-\mu\right)\right]-1} \equiv f\left(\epsilon_{j}\right),
$$

where $\beta=\left(k_{B} T\right)^{-1}$ is proportional to the inverse temperature. Here, $f(\epsilon)=\{\exp [\beta(\epsilon-\mu)]-1\}^{-1}$ is the usual Bose-Einstein distribution function (it depends explicitly on $T$ and $\mu$ ), as shown, for example, in Sec. 5 of Fetter and Walecka (2003), Chap. V of Lifshitz and Pitaevskii (1980a), Part 1, or Chap. 7 of Pathria (1996). A detailed analysis shows that the mean total number of particles $N(T, \mu)$ and the mean total energy $E(T, \mu)$ are given by

$$
\begin{aligned}
& N(T, \mu)=\sum_{j} f\left(\epsilon_{j}\right), \\
& E(T, \mu)=\sum_{j} \epsilon_{j} f\left(\epsilon_{j}\right) .
\end{aligned}
$$

In principle, the first relation Eq. (2.2) can be inverted to give $\mu(T, N)$, and substitution into Eq. (2.3) then yields the mean total energy $E(T, N)$ as a function of temperature and the total number of particles.

It is useful to introduce the "density of states"

$$
g(\epsilon)=\sum_{j} \delta\left(\epsilon-\epsilon_{j}\right)
$$

so that Eqs. (2.2) and (2.3) reduce to

$$
\begin{aligned}
& N(T, \mu)=\int d \epsilon g(\epsilon) f(\epsilon), \\
& E(T, \mu)=\int d \epsilon g(\epsilon) \epsilon f(\epsilon) .
\end{aligned}
$$

Although $g(\epsilon)$ is formally singular, a smoothed (coarsegrained) version would be well-defined.
In the classical limit, the chemical potential $\mu_{\mathrm{cl}}=$ $k_{B} T \ln \left(n \lambda_{T}^{3}\right)$ is large and negative since $n \lambda_{T}^{3} \ll 1$. As the temperature decreases (or the density increases), however, $\mu$ increases toward positive values. The critical temperature $T_{c}$ for the onset of BEC is defined implicitly through the relation $\mu\left(T_{c}, N\right)=\epsilon_{0}$, where $\epsilon_{0}$ is the ground-state energy of the single-particle Hamiltonian with the potential $V_{\mathrm{tr}}$. For $T<T_{c}$, the chemical potential remains fixed at this value, and the ground state has a macroscopic occupation $N_{0}(T)$, whose temperature dependence follows from the conservation of total particles $N=N_{0}(T)+N^{\prime}(T)$, with

$$
N^{\prime}(T)=\int_{\epsilon_{0}}^{\infty} d \epsilon \frac{g(\epsilon)}{\exp \left[\beta\left(\epsilon-\epsilon_{0}\right)\right]-1}
$$

defining the total number of particles not in the condensate. If $g\left(\epsilon_{0}\right)$ vanishes, then this integral is finite and well defined, and the number of noncondensed particles decreases with decreasing temperature. In contrast, if $g\left(\epsilon_{0}\right)$ is finite or singular, then this integral for the number of noncondensed particles diverges, and the system cannot form a BEC. It is helpful to examine two specific examples.

\section{Ideal BEC in a box with periodic boundary conditions}

The textbook example of BEC is an ideal gas in a three-dimensional cubical box of volume $V=L^{3}$ with periodic boundary conditions. The single-particle eigenfunctions are plane waves $\psi_{\boldsymbol{k}}(\boldsymbol{r})=V^{-1 / 2} \exp (i \boldsymbol{k} \cdot \boldsymbol{r})$ with single-particle energy $\epsilon_{k}=\hbar^{2} k^{2} /(2 M)$, where $\boldsymbol{k}=$ $(2 \pi / L)\left(n_{x}, n_{y}, n_{z}\right)$ with $n_{j}$ any integer. It is not hard to obtain the corresponding density of states

$$
g(\epsilon)=\frac{V}{4 \pi^{2}}\left(\frac{2 M}{\hbar^{2}}\right)^{3 / 2} \epsilon^{1 / 2} .
$$

In a box with periodic boundary conditions, the lowest single-particle energy is $\epsilon_{0}=0$, which corresponds to $\boldsymbol{k}=\mathbf{0}$ (a uniform state with density $\left|\psi_{\mathbf{0}}\right|^{2}=V^{-1}$ ). A standard analysis with the conventional definition $\lambda_{T}^{2}=$ $2 \pi \hbar^{2} /\left(M k_{B} T\right)$ yields the condition

$$
n \lambda_{T_{c}}^{3}=\zeta\left(\frac{3}{2}\right) \approx 2.612,
$$

which is the critical phase-space density for the onset of BEC. Alternatively, the critical temperature $T_{c}$ is given by

$$
k_{B} T_{c}=\frac{2 \pi}{\left[\zeta\left(\frac{3}{2}\right)\right]^{2 / 3}} \frac{\hbar^{2} n^{2 / 3}}{M} \approx 3.31 \frac{\hbar^{2} n^{2 / 3}}{M},
$$

as anticipated from the previous qualitative discussion. Below $T_{c}$, the (uniform) condensate with zero momentum has a macroscopic occupation number, which is the signature of BEC. In particular, it is easy to verify that

$$
\frac{N^{\prime}(T)}{N}=\left(\frac{T}{T_{c}}\right)^{3 / 2}
$$


for $T<T_{c}$, so that the macroscopic occupation of the uniform ground state has the temperature dependence

$$
\frac{N_{0}(T)}{N}=1-\left(\frac{T}{T_{c}}\right)^{3 / 2} .
$$

It is worth generalizing these results to a uniform ideal Bose gas in a $d$-dimensional hypercubical box; the corresponding density of states $g(\epsilon)$ is proportional to $\epsilon^{d / 2-1}$. This alteration means that the integral in Eq. (2.7) diverges for $d \leq 2$. As a result, the limit $\mu \rightarrow \epsilon_{0}$ requires a more careful analysis, leading to the conclusion that such a uniform gas in two or one dimension cannot form a BEC; for a detailed treatment of the two-dimensional uniform Bose gas, see Chap. 2 of Pethick and Smith (2002).

\section{Ideal BEC in a harmonic trap}

In three dimensions, an anisotropic harmonic trap potential

$$
V_{\mathrm{tr}}(\boldsymbol{r})=\frac{1}{2} M\left(\omega_{x}^{2} x^{2}+\omega_{y}^{2} y^{2}+\omega_{z}^{2} z^{2}\right)
$$

yields single-particle energies that are labeled by a triplet of non-negative integers $n_{x}, n_{y}, n_{z}$

$$
\epsilon_{n_{x}, n_{y}, n_{z}}=\hbar\left(n_{x} \omega_{x}+n_{y} \omega_{y}+n_{z} \omega_{z}\right)+\epsilon_{0},
$$

where the second term is the zero-point energy $\epsilon_{0}=$ $\frac{1}{2} \hbar\left(\omega_{x}+\omega_{y}+\omega_{z}\right)$. The ground-state wave function is a product of three one-dimensional Gaussians with mean widths $d_{j}=\sqrt{\hbar /\left(M \omega_{j}\right)}$ (here $j=x, y$, or $z$ ). If the sums in the density of states are approximated by integrals (which holds for large $\epsilon$ ), the corresponding density of states is

$$
g(\epsilon)=\frac{\epsilon^{2}}{2 \hbar^{3} \omega_{0}^{3}},
$$

where $\omega_{0}^{3}=\omega_{x} \omega_{y} \omega_{z}$ defines a geometric-mean trap frequency.

The onset of BEC in a harmonic trap occurs at $\mu=\epsilon_{0}$, and an elementary analysis yields the transition temperature

$$
k_{B} T_{c}=[\zeta(3)]^{-1 / 3} \hbar \omega_{0} N^{1 / 3} \approx 0.94 \hbar \omega_{0} N^{1 / 3},
$$

where $\zeta(3) \approx 1.212$. Below $T_{c}$, a macroscopic number of particles occupies the anisotropic Gaussian ground state of this harmonic trap, which has a macroscopic occupation with temperature dependence

$$
\frac{N_{0}(T)}{N}=1-\left(\frac{T}{T_{c}}\right)^{3}
$$

Images of this temperature-dependent Gaussian condensate density profile provided the first clear evidence for the creation of BEC in ${ }^{87} \mathrm{Rb}$ (Anderson et al., 1995). Typical traps have $d_{0}=\sqrt{\hbar /\left(M \omega_{0}\right)} \sim$ a few $\mu \mathrm{m}$ and
$N \sim 10^{6}$, confirming the previous value $T_{c} \approx 100-1000$ $\mathrm{nK}$ (depending on the atomic mass).

For a harmonic trap in $d$ dimensions, the density of states $g(\epsilon)$ is proportional to $\epsilon^{d-1}$, which differs from the $d$ dependence for a hypercubical box. As a result, a two-dimensional Bose gas in a trap can form a BEC with a finite transition temperature $T_{c}$, in contrast to a uniform two-dimensional gas in a box. This behavior is especially important in the limit of rapidly rotating Bose-Einstein condensate, when the centrifugal forces flatten the atomic system, producing an effectively two-dimensional trapped gas. Chapter 2 of Pethick and Smith (2002) discusses this situation in some detail, especially the behavior in a one-dimensional trap, where the order of limits $N \rightarrow \infty$ and $T \rightarrow 0$ becomes crucial.

\section{B. Inclusion of interparticle interactions}

Roughly sixty years ago, Bogoliubov (1947) introduced what is now seen as the essential physical approximation for a dilute Bose gas with repulsive interactions. To understand his idea, note that the repulsive interparticle interaction can be characterized by the positive $s$ wave scattering length $a$, which is typically a few $\mathrm{nm}$ for the dilute alkalai-metal atoms of interest [see Chap. 5 of Pethick and Smith (2002)]. In the low-density limit $\left(a \ll n^{-1 / 3}\right)$, the "gas parameter" $n a^{3}$ is small and provides a natural expansion parameter. Bogoliubov notes that the ground state of the dilute gas will then be close to that of the ideal gas. Thus the number of particles $N_{0}$ in the condensate will be close to the total number $N$, and the difference can be ignored in first approximation. The macroscopic occupation of the single-particle mode for the condensate means that the creation and annihilation operators for this particular mode can be approximated as "classical fields" similar to the electric fields for a laser mode. Bogoliubov's seminal physical picture underlies almost all the subsequent developments in the field and is basic for understanding the behavior of a low-temperature BEC.

For a uniform Bose gas at rest, the condensate is the zero-momentum state, and the excited states are labeled by wavenumber $\boldsymbol{k} \neq \mathbf{0}$, as discussed, for example, in Sec. 25 of Lifshitz and Pitaevskii (1980b), in Sec. 7.2 of Pethick and Smith (2002), and Secs. 18 and 35 of Fetter and Walecka (2003). In the present case of a trapped condensate, however, the analysis is somewhat more intricate and relies on the macroscopic condensate wave function $\Psi$ (sometimes called the "order parameter"). This condensate wave function describes the single-particle mode that has macroscopic occupation, with its squared absolute value $|\Psi(\boldsymbol{r})|^{2}=n(\boldsymbol{r})$ giving the nonuniform condensate particle density $n(\boldsymbol{r})$. For a dilute gas at low temperature with $N_{0} \approx N$, the nor- 
malization requires

$$
\int d V|\Psi(\boldsymbol{r})|^{2}=N_{0} \approx N
$$

In the limit of a nearly ideal Bose gas at $T=0 \mathrm{~K}$, the spatial form of this condensate wave function follows by minimizing the total energy $E$, along with the constraint that the total number of particles $N$ is conserved. Equivalently, one can simply minimize the grand-canonical thermodynamic potential $E-\mu N$, where $\mu$ is the chemical potential. To understand this approach, I shall generally rely on physical arguments, but many-body quantum field theory provides a rigorous foundation for the same results (Fetter, 1972, 1996; Hohenberg and Martin, 1965). In a trap with confining potential $V_{\mathrm{tr}}$, the GrossPitaevskii (Gross, 1961; Pitaevskii, 1961) energy functional for the condensate has the form

$$
E_{G P}[\Psi]=\int d V(\underbrace{\frac{\hbar^{2}|\nabla \Psi|^{2}}{2 M}}_{\text {kinetic }}+\underbrace{V_{\mathrm{tr}}|\Psi|^{2}}_{\text {trap }}+\underbrace{\frac{1}{2} g|\Psi|^{4}}_{\text {interaction }})
$$

containing the kinetic energy, the trap energy [see Eq. (2.13)], and the interaction energy, respectively. The first two terms (those quadratic in $\Psi$ ) are just the onebody energy for an ideal Bose gas in a (usually) harmonic trap. In contrast, the quartic term (the two-body energy) describes the effect of interactions, where the interparticle potential has been approximated by a shortrange "pseudopotential" $V\left(\boldsymbol{r}-\boldsymbol{r}^{\prime}\right) \approx g \delta^{(3)}\left(\boldsymbol{r}-\boldsymbol{r}^{\prime}\right)$, with $g=4 \pi a \hbar^{2} / M$ a coupling constant fixed by the $s$-wave scattering length $a$ [this result follows from standard twobody quantum-mechanical scattering theory, for example, Chap. 5 of Pethick and Smith (2002) or Secs. 11 and 35 of Fetter and Walecka (2003)].

Comparison of the kinetic and trap energies for a harmonic potential yields the familiar oscillator length

$$
d_{0}=\sqrt{\frac{\hbar}{M \omega_{0}}}
$$

that characterizes the mean size of the noninteracting condensate, with $\omega_{0}=\left(\omega_{x} \omega_{y} \omega_{z}\right)^{1 / 3}$. Similarly, comparison of the kinetic energy and the interaction energy for a uniform condensate yields the "healing length"

$$
\xi=\frac{\hbar}{\sqrt{2 M g n}}=\frac{1}{\sqrt{8 \pi a n}},
$$

which characterizes the length scale over which a localized alteration in the condensate density heals back to its uniform value $n$. In this context, recall the Bogoliubov approximation of small quantum depletion $N^{\prime} \ll N$, which requires $n a^{3} \ll 1$. As a result, Eq. (2.21) has the important corollary

$$
n^{2 / 3} \xi^{2}=\frac{1}{8 \pi n^{1 / 3} a} \gg 1
$$

namely the healing length must exceed the interparticle spacing in any GP description.

Variation of $E_{G P}[\Psi]$ in Eq. (2.19) with respect to $\Psi^{*}$ at fixed normalization yields the time-independent GrossPitaevskii (GP) equation (Gross, 1961; Pitaevskii, 1961)

$$
\left[-\frac{\hbar^{2} \nabla^{2}}{2 M}+V_{\operatorname{tr}}(\boldsymbol{r})+g|\Psi(\boldsymbol{r})|^{2}\right] \Psi(\boldsymbol{r})=\mu \Psi(\boldsymbol{r}),
$$

Here the chemical potential $\mu$ can either be considered a Lagrange multiplier or a parameter in the zerotemperature grand-canonical thermodynamic potential $E-\mu N$. In the present context, this GP equation is essentially a nonlinear Schrödinger equation that includes an additional quadratic selfcoupling $g|\Psi|^{2}$; such a term can be interpreted as a Hartree potential $V_{H}(\boldsymbol{r})=g n(\boldsymbol{r})$ that represents the interaction with the local nonuniform condensate density.

Compared to a uniform BEC, a trapped condensate involves an additional characteristic length $d_{0}$ (the noninteracting condensate size). A simple scaling argument [see Sec. 6.2 of Pethick and Smith (2002)] yields a new dimensionless parameter $N a / d_{0}$ that characterizes the importance of the interaction in a trapped condensate. As a result, the three terms in Eq. (2.19) can have very different magnitudes depending on the parameters. In the usual situation $\left(N \approx 10^{6}, a \approx\right.$ a few $\mathrm{nm}$, and $d_{0} \approx \mathrm{a}$ few $\mu \mathrm{m})$, this dimensionless parameter $N a / d_{0}$ is large, and the resulting regime is known as the "Thomas-Fermi limit" (Baym and Pethick, 1996). ${ }^{1}$ In this case, the repulsive interactions dominate and expand the condensate to a mean radius $R_{0}$ that greatly exceeds the mean oscillator length $d_{0}$ (a factor of 10 is typical). This expansion dramatically reduces the radial gradient of the density, and the associated kinetic energy thus becomes negligible relative to the trap energy and the interaction energy. Equation (2.19) then reduces to

$$
E_{\mathrm{TF}}[\Psi] \approx \int d V\left(V_{\mathrm{tr}}|\Psi|^{2}+\frac{1}{2} g|\Psi|^{4}\right)
$$

which involves only $|\Psi|^{2}$ and $|\Psi|^{4}$. Minimization with respect to $|\Psi|^{2}$ at fixed normalization gives the ThomasFermi (TF) approximation

$$
V_{\mathrm{tr}}(\boldsymbol{r})+g|\Psi(\boldsymbol{r})|^{2}=\mu
$$

which also follows by omitting the kinetic energy term in Eq. (2.23). This algebraic equation can be solved directly for the equilibrium density

$$
n(\boldsymbol{r})=\frac{\mu-V_{\mathrm{tr}}(\boldsymbol{r})}{g} \theta\left[\mu-V_{\mathrm{tr}}(\boldsymbol{r})\right],
$$

\footnotetext{
1 As seen below, the limit $N a / d_{0} \gg 1$ means that the condensate density is effectively uniform on relevant length scales, which explains the name "Thomas-Fermi."
} 
where $\theta(x)$ is the unit positive step function. Although this expression holds for any reasonable trap potential, the usual case of a quadratic harmonic trap (2.13) yields the simple and explicit expression

$$
n(\boldsymbol{r})=n(0)\left(1-\frac{x^{2}}{R_{x}^{2}}-\frac{y^{2}}{R_{y}^{2}}-\frac{z^{2}}{R_{z}^{2}}\right)
$$

where the right-hand side is positive and zero otherwise. Here $n(0)=\mu / g$ is the central density and $R_{j}^{2}=$ $2 \mu /\left(M \omega_{j}^{2}\right)$ are the squared condensate radii in the three coordinate directions. This TF density has a parabolic cross section and fills the interior of an ellipsoid.

Application of the normalization condition (2.18) to the TF density (2.27) yields $N=8 \pi n(0) R_{0}^{3} / 15$, where $R_{0}^{3} \equiv R_{x} R_{y} R_{z}$ depends on the chemical potential $\mu$. Some algebra shows that $n(0)=\mu / g=R_{0}^{2} /\left(8 \pi a d_{0}^{4}\right)$, and a combination of these results yields the important dimensionless relation

$$
\frac{R_{0}^{5}}{d_{0}^{5}}=15 \frac{N a}{d_{0}},
$$

which is large in the present TF limit. Correspondingly, the TF chemical potential becomes

$$
\mu_{\mathrm{TF}}=\frac{1}{2} M \omega_{0}^{2} R_{0}^{2}=\frac{1}{2} \hbar \omega_{0} \frac{R_{0}^{2}}{d_{0}^{2}},
$$

so that $\mu_{\mathrm{TF}} \gg \hbar \omega_{0}$ in the TF limit. Since $\mu_{\mathrm{TF}}$ is proportional to $N^{2 / 5}$, the thermodynamic relation $\mu=\partial E / \partial N$ yields the TF energy for a trapped condensate

$$
E_{\mathrm{TF}}=\frac{5}{7} \mu_{\mathrm{TF}} N,
$$

which also follows by direct integration of Eq. (2.24) for the TF density profile.

It is conventional to use the central density $n(0)$ to define the healing length [Eq. (2.21)] in a nonuniform trapped condensate, which gives

$$
\xi^{2}=\frac{1}{8 \pi a n(0)} .
$$

These relations lead to the important conclusion that $\xi^{2}=d_{0}^{4} / R_{0}^{2}$; equivalently,

$$
\frac{\xi}{d_{0}}=\frac{d_{0}}{R_{0}}
$$

This TF limit holds when the mean condensate radius $R_{0}$ is large compared to the mean oscillator length $d_{0}$. Thus the TF oscillator length $d_{0}$ is the geometric mean of $\xi$ and $R_{0}$, and Eq. (2.32) then yields a clear separation of TF length scales, with $\xi \ll d_{0} \ll R_{0}$.

Equation (2.23) has the expected time-dependent generalization (known as the time-dependent GP equation)

$$
i \hbar \frac{\partial \Psi(\boldsymbol{r}, t)}{\partial t}=\left[-\frac{\hbar^{2} \nabla^{2}}{2 M}+V_{\mathrm{tr}}(\boldsymbol{r})+g|\Psi(\boldsymbol{r}, t)|^{2}\right] \Psi(\boldsymbol{r}, t),
$$

where $\Psi$ now depends on $t$ as well as on $\boldsymbol{r}$. Although I shall not discuss the field-theoretical derivation of the time-dependent GP equation, it is worth noting that the condensate wave function is interpreted as an ensemble average $\Psi(\boldsymbol{r}, t)=\langle\hat{\psi}(\boldsymbol{r}, t)\rangle$ of the field operator $\hat{\psi}(\boldsymbol{r}, t)$ Fetter, 1972, 1996; Gross, 1961; Hohenberg and Martin, 1965; Pitaevskii, 1961). Comparison of Eqs. (2.23) and (2.33) implies that a stationary solution has the time dependence $\exp (-i \mu t / \hbar)$. This result also follows directly from the field-theoretical definition because the states on the left-hand side of the ensemble average have one less particle than those on the right-hand side.

This nonlinear field equation (2.33) can be recast in an intuitive hydrodynamic form by writing the condensate wave function

$$
\Psi(\boldsymbol{r}, t)=|\Psi(\boldsymbol{r}, t)| \exp [i S(\boldsymbol{r}, t)]
$$

in terms of its magnitude $|\Psi|$ and phase $S$. In this picture, the condensate (particle) density is $n(\boldsymbol{r}, t)=$ $|\Psi(\boldsymbol{r}, t)|^{2}$, whereas the particle-current density becomes

$$
\boldsymbol{j}=\frac{\hbar}{2 M i}\left(\Psi^{*} \nabla \Psi-\Psi \nabla \Psi^{*}\right)=|\Psi|^{2} \frac{\hbar \nabla S}{M} .
$$

The usual hydrodynamic relation $\boldsymbol{j}=n \boldsymbol{v}$ identifies the local velocity as

$$
\boldsymbol{v}(\boldsymbol{r}, t)=\frac{\hbar}{M} \nabla S(\boldsymbol{r}, t)=\nabla \Phi(\boldsymbol{r}, t),
$$

where $\Phi \equiv \hbar S / M$ is the velocity potential, as proposed by Fevnman (1955) in his discussion of the quantum mechanics of vortices in superfluid He II. Note that $\boldsymbol{\nabla} \times \boldsymbol{v}$ vanishes wherever $S$ is not singular, so that the "superfluid velocity" $\boldsymbol{v}$ is irrotational, as assumed by Landau (1941) in his phenomenological two-fluid hydrodynamics for superfluid He II. For more complete discussions, see, for example, Chap. III of Lifshitz and Pitaevskii (1980b) and Chap. XVI of Landau and Lifshitz (1987).

The representation of the velocity $\boldsymbol{v}$ in terms of the gradient of the phase has one major implication. Consider the "circulation" $\kappa$, defined at a given instant of time in terms of a line integral $\kappa=\oint_{\mathcal{C}} d \boldsymbol{l} \cdot \boldsymbol{v}$ around a closed path $\mathcal{C}$. Substitution of Eq. (2.36) yields

$$
\kappa=\frac{\hbar}{M} \oint_{\mathcal{C}} d \boldsymbol{l} \cdot \nabla S=\left.\frac{\hbar}{M} \Delta S\right|_{\mathcal{C}},
$$

where $\left.\Delta S\right|_{\mathcal{C}}$ is the net change in the phase on following $\mathcal{C}$. The single-valuedness of the condensate wave function requires that this quantity must be an integral multiple of $2 \pi$, which shows that the circulation in a dilute BEC must be quantized in units of $2 \pi \hbar / M$ (Fevnman, 1955; Onsager, 1949). For accounts of this remarkable quantum-mechanical relation, see Sec. 23 of London (1954) and Sec. 2.3 of Donnelly (1991).

Substitute Eq. (2.34) into the time-dependent GP equation (2.33). The imaginary part yields the familiar 
continuity equation for a compressible fluid

$$
\frac{\partial n}{\partial t}+\nabla \cdot(n \boldsymbol{v})=0
$$

Correspondingly, the real part provides a generalized Bernoulli equation for this quantum fluid

$$
\frac{1}{2} M v^{2}+V_{\mathrm{tr}}-\frac{\hbar^{2}}{2 M \sqrt{n}} \nabla^{2} \sqrt{n}+g n+M \frac{\partial \Phi}{\partial t}=0,
$$

where the explicitly quantum-mechanical term containing $\hbar^{2}$ is sometimes called a "quantum pressure." The full structure of this Bernoulli equation (including the quantum pressure) can be understood as that for an irrotational compressible isentropic fluid [see Sec. 2 of Landau and Lifshitz (1987)] with an enthalpy density obtained from Eq. (2.19) (Fetter, 2002). Such an isentropic picture is clearly appropriate for a low-temperature superfluid.

The existence of a Bernoulli equation for the timedependent GP equation has one basic consequence in the present context of vortices in dilute quantum gases. All the classical results about vortex dynamics in an irrotational fluid [such as Kelvin's theorem on the conservation of circulation, proved, for example, in Sec. 8 of Landau and Lifshitz (1987)] automatically hold for any vortex configuration in a dilute BEC. Nevertheless, the presence of a nonuniform trapping potential $V_{\mathrm{tr}}(\boldsymbol{r})$ and the resulting nonuniform density in Eq. (2.26) significantly affect the dynamics of vortices, so that qualitative classical pictures based on uniform incompressible fluids may not always apply.

\section{Bogoliubov equations}

The time-dependent GP equation (2.33) describes the dynamics of the condensate, and it is natural to consider the linearized behavior of small perturbations around such solutions $\Psi(\boldsymbol{r}, t)$. Although a fully quantummechanical analysis is feasible (Bogoliubov, 1947; Fetter, 1972; Pitaevskii, 1961), I here rely on a simpler classical approach (Dalfovo et al., 1999) [see also Sec. 7.2 of Pethick and Smith (2002)] that yields the same eigenvalue equations. Assume a stationary nonuniform condensate with wave function $\Psi(\boldsymbol{r}) e^{-i \mu t / \hbar}$. Since the nonlinear Hartree term involves $|\Psi|^{2}$, the perturbations must, for consistency, include $e^{\mp i \omega t}$ with both signs of the frequency. Specifically, take

$$
\Psi(\boldsymbol{r}, t)=e^{-i \mu t / \hbar}\left[\Psi(\boldsymbol{r})+u(\boldsymbol{r}) e^{-i \omega t}-v^{*}(\boldsymbol{r}) e^{i \omega t}\right],
$$

where the Bogoliubov amplitudes $u(\boldsymbol{r})$ and $v(\boldsymbol{r})$ are treated as small.

Substitute (2.40) into Eq. (2.33) and collect first-order terms proportional separately to $e^{\mp i \omega t}$. The result is a pair of linear eigenvalue equations

$$
\begin{aligned}
\mathcal{L} u_{j}-g(\Psi)^{2} v_{j} & =\hbar \omega_{j} u_{j}, \\
\mathcal{L} v_{j}-g\left(\Psi^{*}\right)^{2} u_{j} & =-\hbar \omega_{j} v_{j},
\end{aligned}
$$

where $\mathcal{L}=-\hbar^{2} \nabla^{2} /(2 M)+V_{\mathrm{tr}}-\mu+2 g|\Psi|^{2}$ is a Hermitian operator. These coupled equations are known as the Bogoliubov equations, although Pitaevskii (1961) was the first to consider the application to nonuniform systems [sometimes they are called the Bogoliubov-de Gennes equations because of their close correspondence to similar equations in the theory of superconductivity, as discussed in Chap. 5 of de Gennes (1966) and Sec. 10.1 of Tinkham (1996)]. In many ways, the Bogoliubov equations are analogous to a nonrelativistic version of the Dirac equation, with $u$ and $v$ as the particle and hole amplitudes, including the $(+,-)$ metric seen in the minus sign on the right side of Eq. (2.42) compared to that of Eq. (2.41) [see, for example, Chap. 12 of Abers (2004), Sec. 52 of Schiff (1968), and Chap. 20 of Shankar (1994)].

For a uniform condensate, the appropriate eigenfunctions are plane waves labeled by $\boldsymbol{k}$. The Bogoliubov energy eigenvalue is easily determined to be

$$
E_{k}=\left[\frac{g n \hbar^{2} k^{2}}{M}+\left(\frac{\hbar^{2} k^{2}}{2 M}\right)^{2}\right]^{1 / 2}
$$

where $n$ is the condensate density and $\mu \approx g n$ for this uniform gas. For long wavelengths $(k \xi \ll 1)$, where $\xi$ is the healing length from Eq. (2.21), the Bogoliubov energy reduces to a phonon spectrum

$$
E_{k} \approx \hbar s k
$$

with $s=\sqrt{g n / M}$ the speed of compressional sound. For short wavelengths $(k \xi \gg 1)$, in contrast, the Bogoliubov spectrum becomes that of a free particle. The existence of a linear (phonon) spectrum at long wavelengths is crucial for superfluidity because the Landau (1941) critical velocity $v_{c}$ for onset of dissipation is the minimum value of $E_{k} /(\hbar k)$ with respect to $k$. In the present case, the Bogoliubov excitation spectrum (2.43) yields $v_{c}=s$. Note that $s \propto \sqrt{g}$ is real and positive only because of the repulsive interactions $(g>0)$. Furthermore, $v_{c}=s$ vanishes for an ideal Bose gas with $g=0$. Hence the ideal Bose gas is not strictly superfluid even at zero temperature, although it does indeed have a condensate with $\boldsymbol{k}=\mathbf{0}$. In this well-defined sense, the existence of superfluidity in a uniform dilute Bose gas arises from the repulsive interactions.

Manipulation of the Bogoliubov equations shows that $\omega_{j} \int d V\left(\left|u_{j}\right|^{2}-\left|v_{j}\right|^{2}\right)$ is real. In most cases, the integral is nonzero, so that $\omega_{j}$ itself is real. To understand the basic issue involved in the normalization of the Bogoliubov eigenfunctions, it helps briefly to recall the usual particle creation and annihilation operators $a_{j}^{\dagger}$ and $a_{j}$ from quantum field theory. These particle and hole operators obey the familiar Bose-Einstein commutation relations $\left[a_{j}, a_{j^{\prime}}^{\dagger}\right]=\delta_{j, j^{\prime}}$. The solution of the Bogoliubov equations constitutes a canonical transformation to a new set of "quasiparticle" operators $\alpha_{j}^{\dagger}$ and $\alpha_{j}$ that are linear combinations of the original particle and hole operators. The situation is very similar to that for BCS superconductors, as shown, for example, in Sec. 5-1 of de Gennes 
(1966) or Sec. 3.5 of Tinkham (1996). Almost by definition, any canonical transformation preserves the commutation relations, so that the Bogoliubov quasiparticle operators must obey the same Bose-Einstein commutation relations $\left[\alpha_{j}, \alpha_{j^{\prime}}^{\dagger}\right]=\delta_{j, j^{\prime}}$. A detailed analysis shows that physically relevant Bogoliubov eigenfunctions must satisfy the following positive normalization condition

$$
\int d V\left(\left|u_{j}\right|^{2}-\left|v_{j}\right|^{2}\right)=1
$$

Under this canonical transformation, the Bogoliobov approximate grand-canonical Hamiltonian assumes the very simple form

$$
H-\mu N \approx \text { const. }+\sum_{j}^{\prime} \hbar \omega_{j} \alpha_{j}^{\dagger} \alpha_{j},
$$

where the sum runs over all states with positive normalization. ${ }^{2}$ It is clear that the sign of the eigenfrequencies is crucial for the stability of the system. If all the frequencies $\omega_{j}$ are positive, then creation of quasiparticles raises the energy, and the system is manifestly stable. If, however, any of the positive-norm solutions has a negative frequency, then creation of those particular quasiparticles lowers the energy, and the system is said to display an "energy instability."

A simple example of this situation is a uniform condensate that moves with velocity $\boldsymbol{v}=\hbar \boldsymbol{q} / M$. The condensate wave function now has a nonuniform complex structure $\Psi(\boldsymbol{r})=\sqrt{n} e^{i \boldsymbol{q} \cdot \boldsymbol{r}}$, and the solution with positive normalization has the excitation energy (Fetter, 1972; Pollock, 1967; Rokhsar, 1997a)

$$
E_{\boldsymbol{k}}(\boldsymbol{v})=\hbar \boldsymbol{k} \cdot \boldsymbol{v}+E_{k},
$$

where $E_{k}$ is the Bogoliubov energy (2.43) for a stationary condensate. For small $v$, this excitation energy is positive for any $k$. When $v$ exceeds the speed of sound $s$, however, then this eigenvalue will be negative in certain directions, which represents the onset of the Landau instability associated with spontaneous creation of phonons (Landau, 1941). Although the energy instability implies that the system is formally unstable, the absence of dissipation means that no mechanism exists to support the growth of the unstable process.

\footnotetext{
2 The criterion for inclusion in this sum has been a source of confusion, because every acceptable solution of the Bogoiubov equation $\left\{u_{j}, v_{j}\right\}$ with frequency $\omega_{j}$ and positive normalization has a second "antiparticle" solution $\left\{v_{j}^{*}, u_{j}^{*}\right\}$ with frequency $-\omega_{j}$ and negative normalization. The original study of these relations (Fetter, 1972) incorrectly assumed that the positive-norm solution always had positive energy. This result indeed describes the simplest case of a uniform stationary condensate with planewave eigenfunctions. In contrast, nonuniform condensates, such as one in uniform motion (discussed here) or one containing a vortex [see Sec. III.B.2 and Rokhsar (1997a)] can have physical states with positive normalization and negative frequency. For a more general discussion, see Fetter and Svidzinskv (2001).
}

For some special condensed systems (typically when BEC occurs in an excited single-particle state), the linearized Bogoliubov equations also have zero-norm solutions with $\int d V\left(|u|^{2}-|v|^{2}\right)=0$. In these cases, the frequencies can be (and usually are) complex. Such systems are said to exhibit a "dynamical instability," and the unstable mode(s) will grow exponentially until various nonlinearities limit the evolution. Such dynamically unstable behavior occurs in a condensate with a doubly quantized vortex ( $\mathrm{Pu}$ et al., 1999; Shin et al., 2004), in certain onedimensional solitons (Feder et al., 2000; Fedichev et al., 1999; Muryshev et al., 1999), and in one-dimensional optical lattices (Wu and Niu, 2001).

\section{PHYSICS OF ONE VORTEX (A FEW VORTICES) IN A TRAP}

When the GP equation was developed in 1961 to provide a tractable model for a vortex line in a BEC, rotating superfluid ${ }^{4} \mathrm{He}$ was the only system of experimental interest (even though ${ }^{4} \mathrm{He}$ is a dense highly correlated system). Since 1995, however, dilute ultracold trapped alkalai-metal gases have provided remarkable physical systems that indeed fit the GP picture in considerable detail.

\section{A. One vortex in unbounded condensate}

Gross (1961) and Pitaevskii (1961) introduced the GP equation specifically to describe a single straight vortex in an otherwise uniform dilute Bose-Einstein condensate with bulk particle density $n$. The condensate wave function has the form $\Psi(\boldsymbol{r})=\sqrt{n} \chi(\boldsymbol{r})$, with

$$
\chi(\boldsymbol{r})=e^{i \phi} f(r / \xi)
$$

where $r, \phi$ are two-dimensional plane-polar coordinates and $f \rightarrow 1$ for large $r$. Equation (2.36) gives the circulating velocity of a singly quantized long straight vortex line

$$
\boldsymbol{v}(\boldsymbol{r})=\frac{\hbar}{M r} \hat{\boldsymbol{\phi}}
$$

this hydrodynamic flow has circular streamlines and a magnitude that diverges as $r \rightarrow 0$. The circulation $\kappa=\oint d \boldsymbol{l} \cdot \boldsymbol{v}=2 \pi \hbar / M$ can be transformed with Stokes's theorem as $\int d \boldsymbol{S} \cdot \boldsymbol{\nabla} \times \boldsymbol{v}=2 \pi \hbar / M$, implying a singular localized vorticity at the center of the vortex core

$$
\boldsymbol{\nabla} \times \boldsymbol{v}=\frac{2 \pi \hbar}{M} \delta^{(2)}(\boldsymbol{r}) \hat{\boldsymbol{z}}
$$

The kinetic energy per unit length of the vortex is

$$
\frac{\hbar^{2}}{2 M} \int d^{2} r|\nabla \Psi|^{2}=\frac{\hbar^{2} n}{2 M} \int d^{2} r\left[\left(\frac{d f}{d r}\right)^{2}+\frac{f^{2}}{r^{2}}\right],
$$


where the first term arises from the density variation near the vortex core and the second term $\left[=\frac{1}{2} M \int d^{2} r n|f(r)|^{2} v^{2}\right]$ is the kinetic energy of the circulating flow. With this kinetic-energy functional, the Euler-Lagrange equation yields the nonlinear GP equation for the radial amplitude $f(r)$. The resulting centrifugal barrier forces the amplitude $f(r)$ to vanish linearly for $r \lesssim \xi$, which characterizes the vortex core, and $f(r) \approx 1$ for $r \gg \xi$. In the present dilute limit, Eq. (2.22) shows that the vortex core is significantly larger than the interparticle spacing. In addition, the particle-current density $|\boldsymbol{j}(\boldsymbol{r})|=n|f(r)|^{2} \hbar /(M r)$ vanishes both far from the vortex and at the center of the vortex, with a maximum near $r \approx \xi$. Finally, the speed of sound in a dilute Bose gas can be rewritten $s=\sqrt{g n / M}=\hbar /(\sqrt{2} M \xi)$, so that the circulating flow in Eq. (3.2) becomes supersonic for $r \lesssim \xi$; in this sense, local acoustic cavitation can be considered the source of the vortex core.

In terms of the dimensionless scaled variable $x=r / \xi$, the variational approximation $f_{\mathrm{var}}(x)=$ $x / \sqrt{x^{2}+2}$ (Fetter, 1969) provides a good fit to the numerical solution of the radial GP equation [see, for example, Fig. 9.1 in Sec. 9.2 of Pethick and Smith (2002)]. The additional GP energy per unit length of vortex is $E_{v} \approx\left(\pi \hbar^{2} n / M\right) \ln (1.46 R / \xi)$, where $R$ is a large-distance cutoff. Apart from the additive numerical constant, this result is essentially the integral of $\frac{1}{2} M v^{2} n$ over a large circle of radius $R$ with a short-distance cutoff of order $\xi$ (Ginzburg and Pitaevskii, 1958).

\section{B. One vortex in a trapped condensate}

A classical viscous fluid in a container that rotates with angular velocity $\Omega$ rapidly acquires the same angular velocity because of the microscopically rough walls. From a theoretical view, the laboratory is no longer the appropriate reference frame because the rotating walls are a time-dependent external potential that can do work on the system. Thus it is essential to transform to the frame rotating with the walls, in which case the external potential becomes stationary. Consider an originally static Hamiltonian $H(\boldsymbol{r}, \boldsymbol{p})$ with an external trap potential $V_{\mathrm{tr}}(x, y, z)$ that in general is anisotropic about $\hat{\boldsymbol{\Omega}}$. When the potential rotates with angular velocity $\boldsymbol{\Omega}$, a direct transformation of the Lagrangian from the laboratory frame to the rotating frame eventually yields the Hamiltonian $H^{\prime}\left(\boldsymbol{r}^{\prime}, \boldsymbol{p}^{\prime}\right)$ in the rotating frame

$$
H^{\prime}\left(\boldsymbol{r}^{\prime}, \boldsymbol{p}^{\prime}\right)=H\left(\boldsymbol{r}^{\prime}, \boldsymbol{p}^{\prime}\right)-\boldsymbol{\Omega} \cdot \boldsymbol{L}\left(\boldsymbol{r}^{\prime}, \boldsymbol{p}^{\prime}\right)
$$

where $\boldsymbol{r}^{\prime}$ and $\boldsymbol{p}^{\prime}$ are the coordinates and canonical momenta in the rotating frame. Here, the right-hand side involves the nonrotating Hamiltonian $H\left(\boldsymbol{r}^{\prime}, \boldsymbol{p}^{\prime}\right)$ and angular momentum $\boldsymbol{L}\left(\boldsymbol{r}^{\prime}, \boldsymbol{p}^{\prime}\right)=\boldsymbol{r}^{\prime} \times \boldsymbol{p}^{\prime}$, with the original variables replaced by those in the rotating frame [see Sec. 39 of Landau and Lifshitz (1960) and Sec. 34 of Lifshitz and Pitaevskii (1980a).$^{3}$

\section{Gross-Pitaevskii energy in a trap}

In the context of the GP equation, this transformation to a rotating frame yields a modified energy functional

$$
\begin{aligned}
E^{\prime}[\Psi] & =\int d V\left[\frac{\hbar^{2}}{2 M}|\nabla \Psi(\boldsymbol{r})|^{2}+V_{\mathrm{tr}}(\boldsymbol{r})|\Psi(\boldsymbol{r})|^{2}\right. \\
& \left.+\frac{1}{2} g|\Psi(\boldsymbol{r})|^{4}\right]-\boldsymbol{\Omega} \cdot \int d V \Psi^{*}(\boldsymbol{r}) \boldsymbol{r} \times \boldsymbol{p} \Psi(\boldsymbol{r})
\end{aligned}
$$

where all variables here are in the rotating frame (written without primes for notational simplicity) [compare Eq. (3.5) ]. The minimization of $E^{\prime}[\Psi]$ now explicitly involves the angular velocity, and the last term $(-\boldsymbol{\Omega} \cdot \boldsymbol{L})$ clearly favors states with nonzero (positive) angular momentum.

Most experiments on vortices in rotating dilute BECs occur in the TF regime. Thus I shall concentrate on this situation, which has several simplifying features. Specifically, the dominant repulsive interactions imply that the mean condensate radius $R_{0}$ is large relative to the original mean oscillator length $d_{0}$. Equation (2.32) then implies that the vortex core size $(\sim \xi)$ is smaller than $d_{0}$ by the same ratio $d_{0} / R_{0}$. Thus the presence of a few vortices does not significantly affect the number density, which can be taken as the unperturbed TF shape in Eq. (2.27). In most cases, the condensates are axisymmetric with radial and axial trap frequencies $\omega_{\perp}$ and $\omega_{z}$, so that the nonrotating TF density depends only on $r=\sqrt{x^{2}+y^{2}}$ and $z$.

If an axisymmetric condensate contains a vortex at the center, the general wave function has the form (Dalfovo and Stringari, 1996; Lundh et al., 1997; Sinha, 1997)

$$
\Psi(\boldsymbol{r})=e^{i \phi}|\Psi(r, z)|
$$

with the phase $\phi$ appropriate for a singly quantized vortex. The hydrodynamic velocity is again $\boldsymbol{v}=\hbar \hat{\boldsymbol{\phi}} /(M r)$, as in Eq. (3.2) for a singly quantized vortex in an asymptotically uniform fluid. The resulting centrifugal barrier near the axis of symmetry creates an axial node in the condensate wave function; thus the presence of one vortex fundamentally alters the topology of the condensate density from ellipsoidal to toroidal because of the hole along the vortex core.

It is important to generalize this wave function to an off-center vortex. For simplicity, consider a threedimensional disk-shaped condensate $\left(\omega_{z} \gg \omega_{\perp}\right)$ with radial condensate dimension $R_{\perp}$. In this case, a vortex

\footnotetext{
${ }^{3}$ Note that $H\left(\boldsymbol{r}^{\prime}, \boldsymbol{p}^{\prime}\right)$ is not simply the original Hamiltonian in the laboratory frame because of the primed variables.
} 
at $\boldsymbol{r}_{0}=\left(x_{0}, y_{0}\right)$ will remain essentially straight, and the principal effect of the lateral translation is the altered phase

$$
S(x, y)=\arctan \left(\frac{y-y_{0}}{x-x_{0}}\right),
$$

which is the azimuthal angle around the shifted origin $\boldsymbol{r}_{0}$. In the TF regime, the density is taken to be unchanged (the logarithmically divergent circulating kinetic energy is cut off at the vortex core radius $\xi$ ).

Use the energy functional $E^{\prime}[\Psi]$ in Eq. (3.6) and let $E_{0}^{\prime}$ denote energy of a vortex-free state (it is independent of $\Omega$ ). If $E_{1}^{\prime}\left(r_{0}, \Omega\right)$ is the total GP energy of a single off-center vortex in the rotating frame, then the difference of the two energies $\Delta E^{\prime}\left(r_{0}, \Omega\right)=E_{1}^{\prime}\left(r_{0}, \Omega\right)-$ $E_{0}^{\prime}$ represents the formation energy of the vortex. A straightforward analysis with this model TF wave function (Svidzinsky and Fetter, 2000a) yields

$$
\frac{\Delta E^{\prime}\left(r_{0}, \Omega\right)}{\Delta E^{\prime}(0,0)}=\left(1-\frac{r_{0}^{2}}{R_{\perp}^{2}}\right)^{3 / 2}-\frac{\Omega}{\Omega_{c}}\left(1-\frac{r_{0}^{2}}{R_{\perp}^{2}}\right)^{5 / 2}
$$

where

$$
\Omega_{c}=\frac{5}{2} \frac{\hbar}{M R_{\perp}^{2}} \ln \left(\frac{R_{\perp}}{\xi}\right),
$$

and the detailed form of $\Delta E^{\prime}(0,0)$ is unimportant here. For fixed $\Omega$, this expression gives the energy in the rotating frame $\Delta E^{\prime}\left(r_{0}, \Omega\right)$ as the vortex moves from the center of the condensate $\left(r_{0}=0\right)$ to the edge $\left(r_{0}=R_{\perp}\right)$, shown in Fig. 1 for various fixed values of $\Omega$ Packard and Sanders (1972) make a similar analysis for a vortex in an incompressible uniform superfluid; Castin and Dum (1999) obtain comparable results numerically for a vortex in a trapped condensate].

(a) Curve (a) shows the energy $\Delta E^{\prime}\left(r_{0}, 0\right)$ of a vortex in a nonrotating condensate as it moves from the center to the outer edge. This energy decreases monotonically as $r_{0}$ increases, with negative curvature for $r_{0} \leq R_{\perp} / \sqrt{2}$. If there is no dissipation, the distance $r_{0}$ determines the energy, and the vortex follows a circular trajectory at fixed $r_{0}$. At low but finite temperature, however, weak dissipation slowly reduces the energy of the vortex, which thus moves outward along curve (a), executing a spiral trajectory toward the edge of the condensate (Rokhsar, 1997a, b) .

(b) With increasing $\Omega$, the function $\Delta E^{\prime}\left(r_{0}, \Omega\right)$ for fixed $\Omega$ flattens and the (negative) central curvature decreases. Curve (b) has zero central curvature, which occurs for

$$
\Omega_{m}=\frac{3}{2} \frac{\hbar}{M R_{\perp}^{2}} \ln \left(\frac{R_{\perp}}{\xi}\right)=\frac{3}{5} \Omega_{c} .
$$

This angular velocity $\Omega_{m}$ represents the onset of metastability for a vortex near the center of a rotating condensate. For $\Omega<\Omega_{m}$, the negative central curvature means that weak dissipation moves the vortex away from the center, and it eventually spirals out to the edge. For $\Omega>\Omega_{m}$, however, the central curvature is now positive, and weak dissipation will move the vortex back to the center. In this latter regime, the surrounding barrier makes the vortex locally stable near the trap center, even though it is not globally stable because the energy at the edge is definitely lower than the energy at the center.

(c) Eventually, at $\Omega_{c}$ defined in Eq. (3.10), the central energy $\Delta E^{\prime}\left(0, \Omega_{c}\right)$ vanishes [and thus equals $\left.E^{\prime}\left(R_{\perp}, \Omega_{c}\right)\right]$. For $\Omega>\Omega_{c}$, the central vortex is both locally and globally stable. This value $\Omega_{c}$ is interpreted as the thermodynamic critical angular velocity for the creation of a singly quantized vortex in the TF limit. ${ }^{4}$ As discussed below, experiments on rotating trapped gases generally find a different (larger) critical angular velocity for the creation of a vortex, but similar analyses for incompressible fluids in cylindrical containers do provide a reasonable description of vortex formation in rotating superfluid ${ }^{4} \mathrm{He}$ (Packard and Sanders, 1972; Yarmchuk and Packard, 1982) [see also Secs. 2.5, 5.1 and 5.2 of Donnelly (1991)].

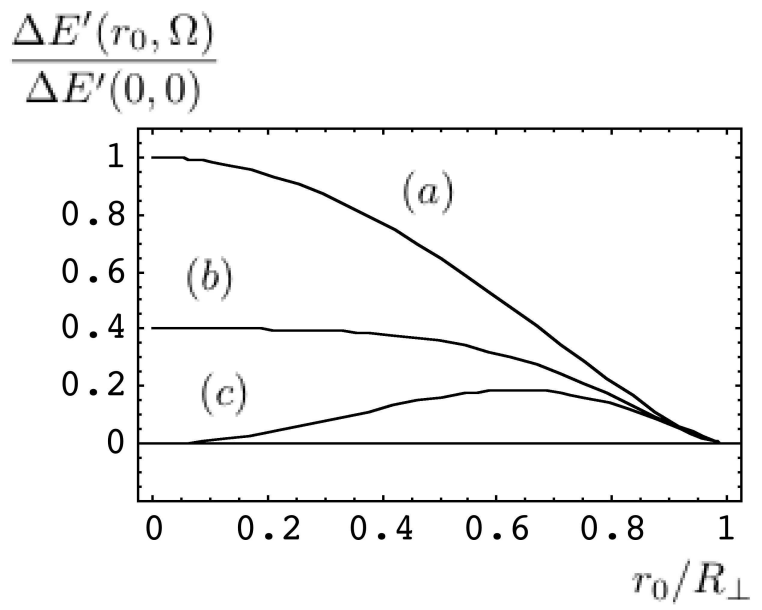

\footnotetext{
${ }^{4}$ For completeness, the corresponding thermodynamic critical angular velocity for a weakly interacting Bose-Einstein gas is $\Omega_{c} / \omega_{\perp} \approx 1-N a /\left(\sqrt{8 \pi} d_{z}\right.$ ) (Butts and Rokhsar, 1999; Linn and Fetter, 1999). Note that this result also holds for attractive interactions $(a<0)$, but the resulting inequality $\Omega_{c}>\omega_{\perp}$ implies that the radial confinement disappears before the vortex would be stable, in agreement with earlier conclusions (Dalfovo and Stringari, 1996; Wilkin et al., 1998). Indeed, attractive interactions quite generally tend to suppress BEC in favor of "fragmented" states with macroscopic occupation in more than one single-particle state (Nozières and Saint James, 1982; Pollock, 1967).
} 
FIG. 1 Dimensionless scaled energy $\Delta E^{\prime}\left(r_{0}, \Omega\right) / \Delta E^{\prime}(0,0)$ [Eq. (3.9)] of a singly quantized straight vortex in a rotating disk-shaped TF trap as a function of the displacement $r_{0}$ from the symmetry axis. Different curves represent different fixed values of the angular velocity $\Omega$ : (a) $\Omega=0$; (b) $\Omega=\Omega_{m}$, defined in Eq. (3.11); (c) $\Omega=\Omega_{c}$, defined in Eq. (3.10).

\section{Dynamical motion of a trapped vortex}

Although the GP energy functional Eq. (3.6) describes the equilibrium of a GP condensate with one vortex (or more vortices), it also provides a very useful dynamical picture of the vortex motion in a trapped condensate.

a. Lagrangian functional The most direct approach relies on the basic observation that the time-dependent GP equation (2.33) is the Euler-Lagrange equation for the time-dependent Lagrangian functional

$$
\mathcal{L}[\Psi]=\int d V \frac{i \hbar}{2}\left(\Psi^{*} \frac{\partial \Psi}{\partial t}-\frac{\partial \Psi^{*}}{\partial t} \Psi\right)-E^{\prime}[\Psi]
$$

under variation of $\Psi^{*}$. If the condensate wave function $\Psi$ depends on one or more parameters, the resulting Lagrangian functional provides approximate Lagrangian equations of motion for these parameters. In the context of BECs, this approach was first applied with great success to the low-lying normal modes of a trapped stationary (nonrotating) condensate (Pérez-García et al., 1996). Subsequently, its application to a single straight vortex in a disk-shaped condensate yields an explicit prediction for the angular precession of such a trapped vortex (Lundh and Ao, 2000; McGee and Holland, 2001; Svidzinsky and Fetter, 2000a). In this case, the position $\boldsymbol{r}_{0}$ of the off-center vortex serves as an appropriate parameter, and the Lagrangian formalism shows that the precession rate is proportional to (the negative of) the slope of the appropriate curve in Fig. 1. For a nonrotating disk-shaped TF condensate, this analysis gives

$$
\dot{\phi}=\frac{\Omega_{m}}{1-r_{0}^{2} / R_{\perp}^{2}}
$$

where $\Omega_{m}=\frac{3}{2} \hbar \ln \left(R_{\perp} / \xi\right) /\left(M R_{\perp}^{2}\right)$ is the critical rotation frequency for the onset of metastability given in Eq. (3.11). Note that a vortex in a nonrotating condensate precesses in the positive sense, which is the same sense as the circulating vortex velocity field.

In this Lagrangian approach, the factor $1-r_{0}^{2} / R_{\perp}^{2}$ in the denominator arises from the parabolic radial TF density profile. It means that a vortex near the outer boundary precesses more rapidly than one near the center, although this simple picture omits bending of the vortex and thus fails as $r_{0} \rightarrow R_{\perp}$.

It is interesting to compare this TF result [Eq. (3.13)] to the precession rate for a long straight classical vortex in an incompressible fluid inside a circular cylinder of radius $R$. In this latter case, the boundary condition of zero radial flow velocity at the radius $R$ requires an external image vortex, yielding the result [see, for example, Sec. 9.4 of Pethick and Smith (2002)]

$$
\dot{\phi}_{\mathrm{cl}}=\frac{\hbar}{M R^{2}} \frac{1}{1-r_{0}^{2} / R^{2}} \text {. }
$$

Although the two expressions for the precession rate have the same denominators, the precession here arises from the motion induced by the image (and there is no large logarithmic factor). Since the TF density necessarily vanishes at the condensate radius, image vortices are generally omitted for trapped condensates, although this question remains partially unresolved (Anglin, 2002; Khawaja, 2005; Mason et al., 2006).

b. Matched asymptotic expansion An alternative approach to the dynamics of a vortex in a trapped condensate is to study the time-dependent GP equation (2.33) itself; this analysis relies on the method of matched asymptotic expansions (Pismen and Rubinstein, 1991; Rubinstein and Pismen, 1994) [see also Secs. 2.2 and 5.2 of Pismen (1999)]. In the TF limit, the vortex core is small compared to other length scales, and it is possible to use the separation of length scales as follows (Svidzinsky and Fetter, 2000b). Assume that the straight vortex (located at $\boldsymbol{r}_{0}$ ) moves with a locally uniform velocity $\boldsymbol{V}\left(\boldsymbol{r}_{0}\right)$ that lies in the $x y$ plane. Transform to a locally comoving reference frame in which the vortex is stationary. The problem is solved approximately in two different regimes:

1. Near the vortex core, the short-distance solution shows that the trap potential exerts a force proportional to $\nabla_{\perp} V_{\mathrm{tr}}$ evaluated at $\boldsymbol{r}_{0}$. This solution includes both the detailed nonuniform structure of the vortex core and the asymptotic region $\xi \ll\left|\boldsymbol{r}-\boldsymbol{r}_{0}\right|$.

2. Far from the vortex, the core can be approximated as a line singularity, and the resulting solution also includes the region $\xi \ll\left|\boldsymbol{r}-\boldsymbol{r}_{0}\right|$.

The two solutions must agree in the common region of overlap, and a lengthy analysis determines the translational velocity $\boldsymbol{V}\left(\boldsymbol{r}_{0}\right)$ of the vortex line near the center of the three-dimensional disk-shaped trap

$$
\boldsymbol{V}\left(\boldsymbol{r}_{0}\right)=\frac{3 \hbar}{4 M \mu} \ln \left(\frac{R_{\perp}}{\xi}\right) \hat{\boldsymbol{z}} \times \nabla_{\perp} V_{\mathrm{tr}}\left(\boldsymbol{r}_{0}\right) .
$$

To understand the structure of this result, note that the trap exerts a force $-\nabla_{\perp} V_{\mathrm{tr}}$ on the vortex. Since the vortex itself has intrinsic angular momentum, it acts gyroscopically and hence moves at right angles to this applied force. As a result, the motion follows an equipotential line along the direction $\hat{\boldsymbol{z}} \times \nabla_{\perp} V_{\mathrm{tr}}$ and thus conserves 
energy, as expected for the GP equation at zero temperature.

In fact, this result is essentially the same as that in Eq. (3.13) for $r_{0}$ near the trap center. The gradient of the axisymmetric potential $V_{\mathrm{tr}}=\frac{1}{2} M\left(\omega_{\perp}^{2} r^{2}+\omega_{z}^{2} z^{2}\right)$ and the squared TF condensate radius $R_{\perp}^{2}=2 \mu /\left(M \omega_{\perp}^{2}\right)$ readily yield the equivalent form for Eq. (3.15)

$$
\boldsymbol{V}\left(\boldsymbol{r}_{0}\right)=\Omega_{m} r \hat{\boldsymbol{\phi}}
$$

involving the critical angular velocity $\Omega_{m}$ for the onset of metastability given in Eq. (3.11). These expressions for the translational velocity $\boldsymbol{V}$ can be generalized to treat a rotating anisotropic harmonic trap with $\omega_{x} \neq$ $\omega_{y}$, and to include the three-dimensional effects of vortex curvature (Svidzinsky and Fetter, 2000a, b).

Although the final result in Eq. (3.15) is simple and physical, the details of the method of matched asymptotic expansions are not very transparent. A more physical picture starts from the conservation of particles $\boldsymbol{\nabla} \cdot(n \boldsymbol{v})=0$ in the locally comoving coordinate system. In the TF limit, the particle density is explicitly known in terms of the trap potential [compare Eq. (2.27)]. Since $\boldsymbol{v} \propto \nabla S$, this equation can be rewritten as $\nabla n \cdot \nabla S+n \nabla^{2} S=0$, which determines the phase $S$ of the condensate wave function in terms of the known density. A perturbation expansion with the appropriate azimuthal angle [see Eq. (3.8)] as the leading term yields an additional phase proportional to $\ln \left|\boldsymbol{r}-\boldsymbol{r}_{0}\right|$ (Sheehy and Radzihovsky, 2004a b; Svidzinsky and Fetter, 2000b). The gradient of this additional phase evaluated at $\boldsymbol{r}_{0}$ then fixes the precessional velocity of the vortex (3.15). In this latter perspective, the precessional motion can be thought to arise from the gradient of the condensate density $n(\boldsymbol{r})$ evaluated at the position of the vortex (Sheehy and Radzihovsky, $2004 \mathrm{a}, \mathrm{b}$ )

$$
\boldsymbol{V}\left(\boldsymbol{r}_{0}\right)=\frac{\hbar}{M} \frac{\hat{\boldsymbol{z}} \times(-\nabla n)}{2 n} \ln \left(\frac{R_{\perp}}{\xi}\right),
$$

where the minus sign appears because the density decreases with increasing $r$ (this detailed result describes a two-dimensional condensate). Apart from a numerical factor that reflects the difference between two and three dimensions, Eqs. (3.15) and 3.17) describe the same physics because the gradient of the TF density $\nabla n$ is proportional to $-\nabla V_{\operatorname{tr}}$ [see Eq. (2.26)].

c. Bogoliubov equations and stability of a vortex Soon after the creation of BECs in dilute ${ }^{87} \mathrm{Rb}$ (Anderson et al., 1995), theorists studied the collective-mode spectrum of a nonrotating stationary vortex-free trapped condensate using the GP equation (2.23) for the condensate and the Bogoliubov equations (2.41) and (2.42) for the smallamplitude normal modes. As Edwards et al. (1996) reported, the agreement between the theoretical predictions and the experimental observations was impressive [see also Sec. IV.A of Dalfovo et al. (1999)].
This success rapidly led to a similar numerical study (Dodd et al., 1997) of the collective modes of a singly quantized vortex in an otherwise nonrotating trapped condensate. If the vortex is on the axis of symmetry, the condensate wave function is like that in Eq. (3.7), and the conservation of the $z$ component of angular momentum greatly simplifies the analysis. Remarkably, the spectrum of quasiparticle excitations for the vortex turns out to contain a very unusual mode (now called "anomalous") with positive normalization integral [Eq. (2.45)], negative angular momentum $m_{a}=-1$ relative to that of the vortex, and negative frequency $\omega_{a}$ Rokhsar (1997b) has emphasized this particular identification; see also Fetter (1998)]. As noted in Sec. II.C [see Eq. (2.46)], such a negative eigenfrequency implies that a vortex in a nonrotating condensate is formally (energetically) unstable, but the vortex cannot spiral out of the condensate in the absence of dissipation [compare the discussion concerning curve (a) in Fig. 1. Detailed studies (Linn and Fetter, 1999; Svidzinsky and Fetter, 2000a) show that $\omega_{a} \approx-\omega_{\perp}$ in the near-ideal limit and $\omega_{a} \approx$ $-\Omega_{m}$ in the TF limit, so that $\omega_{a}$ remains negative for all coupling strengths.

The physics underlying the anomalous mode is especially transparent in the weak-coupling (near-ideal) limit, when the condensate with a singly quantized vortex at the origin has the radial wave function $\psi_{1}(r, \phi) \propto$ $(x+i y) \exp \left(-\frac{1}{2} r^{2}\right)=r e^{i \phi} \exp \left(-\frac{1}{2} r^{2}\right)$, with one unit of angular momentum and an excitation energy $\hbar \omega_{\perp}$. Note that $\psi_{1}$ differs from the true radial ground-state wave function $\psi_{0} \propto \exp \left(-\frac{1}{2} r^{2}\right)$, which instead has zero angular momentum and zero excitation energy. In principle, the particles in the macroscopic vortex condensate could make a transition to the nonrotating ground state, with a change in angular momentum $m=-1$ and a change in energy $-\hbar \omega_{\perp}$; this transition is just the anomalous mode in an ideal Bose-Einstein gas. Such anomalous modes typically occur whenever the condensate occupies a single-particle state that is not the true ground state [compare the discussion of a moving condensate below Eq. (2.47)].

In the present case of a condensate with a single vortex, the anomalous mode has two very important consequences

1. The linearized solution for the $j$ th mode of the time-dependent GP equation in Eq. (2.40) immediately yields the corresponding perturbation in the density $\delta n_{j}=\left(\Psi^{*} u_{j}-\Psi v_{j}\right) e^{-i \omega_{j} t}$. In particular, a singly quantized vortex with $\Psi$ given in Eq. (3.7) has an anomalous-mode density perturbation $\delta n_{a}(\boldsymbol{r}, t) \propto \exp \left[i\left(m_{a} \phi-\omega_{a} t\right)\right]$, with $m_{a}=-1$ and negative excitation frequency $\omega_{a}=-\left|\omega_{a}\right|$. This density perturbation $\delta n_{a} \propto \exp \left[i\left(\left|\omega_{a}\right| t-\phi\right)\right]$ precesses in the positive sense around the symmetry axis of the condensate at a rate $-\omega_{a}=\left|\omega_{a}\right|$. In the TF limit when $\left|\omega_{a}\right|=\Omega_{m}$ (Svidzinsky and Fetter, 2000a), this rate agrees with that in Eq. (3.13) and with experiments (Anderson et al., 2000) discussed 
below in Sec. III.D.1.

2. When the condensate rotates, Eq. (3.5) shows that the frequency $\omega_{j}$ of the $j$ th mode in the laboratory frame shifts to

$$
\omega_{j}^{\prime}(\Omega)=\omega_{j}-m_{j} \Omega
$$

in the rotating frame, where $m_{j}$ is the corresponding angular quantum number. For the anomalous mode, this result implies that $\omega_{a}(\Omega)=-\left|\omega_{a}\right|+\Omega$, which becomes positive and thus energetically stable when $\Omega$ exceeds $\left|\omega_{a}\right|$. This conclusion confirms the critical rotation frequency $\Omega_{m}$ for the onset of metastability in the TF regime, as seen in curve (b) of Fig. 1.

\section{Small vortex arrays in a trap}

In the TF limit, Eq. (3.8) shows that the condensate wave function has a $2 \pi$-phase singularity associated with a vortex at $\boldsymbol{r}_{0}$. More generally, a vortex is simply a node in the condensate wave function (Feynman, 1955). In the context of dilute ultracold BECs, Butts and Rokhsar (1999) exploit this property in their studies of small vortex arrays Castin and Dum (1999) obtain similar vortex configurations with a somewhat different variational approach].

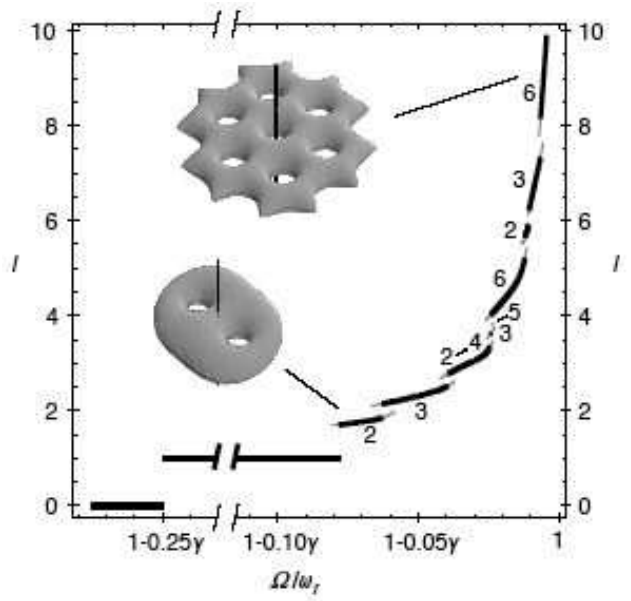

FIG. 2 Angular momentum $l$ vs. dimensionless angular velocity $\Omega / \omega_{\perp}$ for stable (black lines) and metastable (gray lines) states. Here, $\gamma=(2 / \pi)^{1 / 2} N a / d_{z}$ is assumed small. There are no stable states with $0<l<1$, and $l=1$ represents a single vortex on the axis of symmetry. For larger $l$, the angular momentum changes smoothly because the positions of the two or more vortices vary continuously with $\Omega$ (for each stable branch, the integer denotes the rotational symmetry). Three-dimensional plots of the density for two and seven vortices are remarkably similar to experimental images of Yarmchuk et al. (1979) for superfluid ${ }^{4} \mathrm{He}$ (see Fig. 8) and of Madison et al. (2000a) for ultracold dilute ${ }^{87} \mathrm{Rb}$ gas (see Fig. 7). From Butts and Rokhsar (1999).
To understand their procedure, assume that the condensate is tightly confined in the $z$ direction with a small axial oscillator length $d_{z} \ll d_{\perp}$. The remaining twodimensional isotropic harmonic trap has the one-body Hamiltonian $H_{0}=\frac{1}{2}\left(p^{2} / M+M \omega_{\perp}^{2} r^{2}\right)$. For positive angular momentum, the low-lying normalized one-body eigenfunctions are

$$
\begin{aligned}
\psi_{m}(\boldsymbol{r}) & =\frac{u^{m} e^{i m \phi}}{(\pi m !)^{1 / 2} d_{\perp}} \exp \left(-\frac{u^{2}}{2}\right) \\
& =\frac{\zeta^{m}}{(\pi m !)^{1 / 2} d_{\perp}} \exp \left(-\frac{u^{2}}{2}\right),
\end{aligned}
$$

where $u=r / d_{\perp}, \zeta=(x+i y) / d_{\perp}=u e^{i \phi}$, and $m \geq 0$. Note that $\psi_{m}$ is also an eigenfunction of the dimensionless angular-momentum operator $l=-i \partial / \partial \phi$ with eigenvalue $m$. As a simple example of a possible trial state, consider the linear combination $\Psi=c_{0} \psi_{0}+c_{1} \psi_{1}$, which vanishes at $\zeta_{0} \equiv\left(x_{0}+i y_{0}\right) / d_{\perp}=-c_{0} / c_{1}$ (the only node). The point $\zeta_{0}$ is the position of the vortex, since the phase of $\Psi$ increases by $2 \pi$ on going around $\zeta_{0}$ in the positive sense.

For a rotating condensate with positive angular momentum, Butts and Rokhsar (1999) use a general linear combination of such states $\Psi=\sum_{m \geq 0} c_{m} \psi_{m}$ with normalization $\sum_{m \geq 0}\left|c_{m}\right|^{2}=1 .^{5} \quad$ The expectation value of the angular momentum per particle is $\hbar l=$ $\hbar \sum_{m \geq 0} m\left|c_{m}\right|^{2}$. Furthermore, the expectation value of the one-body Hamiltonian $\left\langle H_{0}\right\rangle=\hbar \omega_{\perp} \sum_{m>0} m\left|c_{m}\right|^{2}+$ $\hbar \omega_{\perp}=\hbar \omega_{\perp}(l+1)$ is essentially that of the angular momentum. Thus all states with the same value of $l$ have the same one-body energy, independent of the specific coefficients $c_{m}$. To lift this degeneracy, it is necessary to consider the interaction energy $E_{\text {int }}=\frac{1}{2} g \int d^{3} r|\Psi|^{4}$ (assumed to be small). In the limit of tight $z$ confinement, this energy reduces to a two-dimensional integral, which they evaluate numerically with the linear-combination trial state $\Psi$. For a given angular momentum $l$, the equilibrium state minimizes the total energy, which also means the interaction energy because of the degeneracy. In this way, Butts and Rokhsar (1999) determine the coefficients $c_{m}$ and thus the position of the vortices (the nodes of $\Psi$ ) as a function of $l$

Given the equilibrium energy $E_{\text {tot }}(l)$, the thermodynamic relation $\hbar \Omega=\partial E_{\text {tot }} / \partial l$ fixes the corresponding angular velocity $\Omega$. Extensive numerical studies (Butts and Rokhsar, 1999) then determine the resulting physical sequence of vortex states with increasing $\Omega$, as shown in Fig. 2. The state with $l=1$ arises from a single vortex on the symmetry axis of the condensate, but configurations of two or more vortices vary with $l$, so that the corresponding angular momentum does not generally take integer values.

\footnotetext{
${ }^{5}$ Similar solutions play a crucial role in the study of rapidly rotating condensates in Sec. V.
} 


\section{Experimental and theoretical studies in a trap}

Two basic experimental methods have been most effective in creating vortices in a BEC. The first approach (Matthews et al., 1999b) manipulates two hyperfine components of ${ }^{87} \mathrm{Rb}$, spinning up one component with an external coherent electromagnetic coupling beam (Williams and Holland, 1999). This approach relies heavily on techniques from atomic physics, especially the optical Bloch equations for a two-level system [see, for example, Chaps. 7-11 of Fevnman (1965), Chap. IV of Cohen-Tannoudji et al. (1977), Chaps. 1-3 of Allen and Eberly (1987), and Chap. V of Cohen-Tannoudji et al. (1998)]. The second nearly simultaneous approach (Madison et al., 2000a) is similar to the "rotating-bucket" method of conventional lowtemperature physics [see, for example, Chaps. 2 and 5 of Donnelly (1991)]. In the present context of BECs, a driven rotating deformation of the confining trap serves to spin up the whole condensate.

\section{Vortex behavior in two-component BEC mixtures (JILA)}

The search for quantized vortices in dilute BECs started soon after the creation of trapped condensates. Since a mixture of two hyperfine components of ${ }^{87} \mathrm{Rb}$ play an essential role in the first reported vortex (Matthews et al., 1999b), I first review the physics of such systems. The GP energy functional in Eq. (2.19) is readily generalized for a mixture of two BECs with condensate wave functions $\Psi_{1}$ and $\Psi_{2}$. The total energy contains (1) the two separate energies $E_{j}\left[\Psi_{j}\right]$ of each component $j=1,2$, with appropriate selfinteraction parameters $g_{j}$ (I assume repulsive interactions with $g_{j}>0$ ) and (2) the interaction energy $E_{12}\left[\Psi_{1}, \Psi_{2}\right]=\int d V g_{12}\left|\Psi_{1}\right|^{2}\left|\Psi_{2}\right|^{2}$ between species 1 and 2 , where $g_{12}$ is the interspecies interaction parameter [see Sec. 12.1 of Pethick and Smith (2002) and Sec. 21.11 of Pitaevskii and Stringari (2003)]. For the simplest case of two uniform BECs in a box, if $g_{12}^{2}>g_{1} g_{2}$, the two components are immiscible and separate into nonoverlapping phases. Otherwise, if $g_{12}^{2}<g_{1} g_{2}$, they form two uniform interpenetrating BECs [in the context of dilute BECs, this result apparently was first obtained by Nepomnyashchi (1974); see also (Colson and Fetter, 1978)].

For trapped dilute BECs, the situation is more complicated because the nonuniform trap potentials $V_{j}$ induce both kinetic and potential energies that affect the overall energy functional. The first such experiment (Myatt et al., 1997) at JILA (Boulder, CO) uses two different hyperfine states of ${ }^{87} \mathrm{Rb}$. This isotope has nuclear spin $I=\frac{3}{2}$, which combines with the single valence electron $\left(S=\frac{1}{2}\right)$ to give two hyperfine manifolds, a lower one with $F=1$ and an upper one with $F=2$. The typical trap has a local minimum in the magnetic field, and only some of these hyperfine states are weak-field seeking and hence stable [see
Chaps. 3 and 4 of Pethick and Smith (2002) and Chap. 9 of Pitaevskii and Stringari (2003) for a description of magnetic and optical traps]. In the common notation $\left|F, m_{F}\right\rangle$, the stable weak-field seeking states are $|1,-1\rangle$, $|2,1\rangle$, and $|2,2\rangle$.

The experiment of Mvatt et al. (1997) cools state $|1,-1\rangle$ evaporatively and the interspecies interaction cools state $|2,2\rangle$ through sympathetic cooling. Although the interaction parameters approximately satisfy $g_{12}^{2} \lesssim g_{1} g_{2}$ (Cornell et al., 1998), implying that two such uniform BECs would overlap, the trapped condensates in fact separate because of the differences in the two trap potentials and interaction parameters (Mvatt et al., 1997). A careful theoretical analysis (Esry et al., 1997) that includes all the relevant effects finds a reasonable fit to the experiments.

To avoid the problem of different trap potentials, subsequent experiments on these mixtures use the pair of states $|1\rangle \equiv|1,-1\rangle$ and $|2\rangle \equiv|2,1\rangle$ that differ by an angular momentum $\Delta m=2$. Each component can be imaged selectively with appropriately tuned lasers. Furthermore the state can be quickly changed from $|1\rangle$ to $|2\rangle$ and back by a two-photon transition (microwave at $\approx 6.8 \mathrm{GHz}$ and $\mathrm{rf}$ at $\approx 2 \mathrm{MHz}$ ). To understand the physics of this fascinating system, it is helpful to examine the coupled time-dependent GP equations for the two components (Hall et al., 1998)

$$
\begin{gathered}
i \hbar \frac{\partial}{\partial t}\left(\begin{array}{c}
\Psi_{1} \\
\Psi_{2}
\end{array}\right)= \\
\left(\begin{array}{cc}
\mathcal{T}+V_{1}+V_{H 1}+\frac{1}{2} \hbar \delta & \frac{1}{2} \hbar \Omega(t) e^{i \omega_{\mathrm{em}} t} \\
\frac{1}{2} \hbar \Omega(t) e^{-i \omega_{\mathrm{em}} t} & \mathcal{T}+V_{2}+V_{H 2}-\frac{1}{2} \hbar \delta
\end{array}\right)\left(\begin{array}{l}
\Psi_{1} \\
\Psi_{2}
\end{array}\right)
\end{gathered}
$$

where $\mathcal{T}=-\hbar^{2} \nabla^{2} /(2 M)$ is the kinetic energy, $V_{j}$ is the trap potential, and $V_{H j}$ is the Hartree energy including the interaction with both densities $\left|\Psi_{1}\right|^{2}$ and $\left|\Psi_{2}\right|^{2}$. The crucial new feature here is the applied electromagnetic (microwave and $\mathrm{rf}$ ) fields with combined frequency $\omega_{\mathrm{em}}$; they produce an off-diagonal coupling that involves the phase of each condensate wave function. The effective coupling $\Omega(t)$ is known as the "Rabi" frequency, which increases with the strength of the applied electromagnetic fields [see Ch. 3 of Allen and Eberly (1987)]. In general, this coupling depends explicitly on time (it vanishes when the fields are turned off). Finally, $\delta$ is the detuning between the combined frequencies of the driving fields and that of the atomic hyperfine transition $|1\rangle \leftrightarrow|2\rangle$.

This electromagnetically coupled two-component system provides a remarkable example of topological control of a quantum-mechanical state through an external parameter. Specifically, when the near-resonant electromagnetic coupling $\Omega(t)$ is turned off, the interaction between the condensates involves only the densities (through $V_{H j}$ ) with no phase information. In this case, each condensate has its own complex order parameter $\Psi_{j}$. The associated $U(1)$ symmetry [see, for example, Sec. 27 of Schiff (1968)] has the topology of a circle or a 
cylinder. For $T \ll T_{c}$, the magnitude $\left|\Psi_{j}\right|$ is fixed, and each complex order parameter has its own phase angle, yielding a quantized circulation that remains a topological invariant.

The topology is very different when the electromagnetic coupling is turned on because the coupled condensates in Eq. 3.20) now have a single two-component $S U(2)$ order parameter with the topology of a sphere, like that of a particle with spin $\frac{1}{2}$ [see Sec. 27 of Schiff (1968) and (Hall et al., 1998)]. Two spherical-polar angles suffice to characterize the coupled system, whose dynamics obeys the optical Bloch equations (Allen and Eberly, 1987), similar to those of nuclear magnetic resonance (NMR). Specifically, the transformation of $|1\rangle$ into $|2\rangle$ is analogous to a $\pi$ pulse in NMR that rotates a spin from up to down. If the pulse is twice as long, the resulting $2 \pi$ rotation essentially reproduces the initial $|1\rangle$ state up to a sign.

An elegant experiment (Hall et al., 1998) shows the ability to manipulate the coupled condensates as a single $S U(2)$ order parameter. Application of a $\pi / 2$ pulse to a pure state $|1\rangle$ produces a coherent mixture with equal population in both states that are spatially separated with a small region of overlap. After a variable delay, a second $\pi / 2$ pulse measures the relative quantum phase between them. A subsequent experiment demonstrates the three-dimensional character of the $S U(2)$ topology explicitly (Matthews et al., 1999a; Williams et al., 2000) as follows: An external coupling field with a spatial gradient produces a differential twist along the length of the condensate. With increasing time, the increasing phase difference between the two ends of the condensate relaxes periodically by a three-dimensional evolution of the coupled $S U(2)$ order parameter, leading to a collapse and recurrence of the structure. Note that such behavior would not occur for two uncoupled $U(1)$ condensate wave functions.

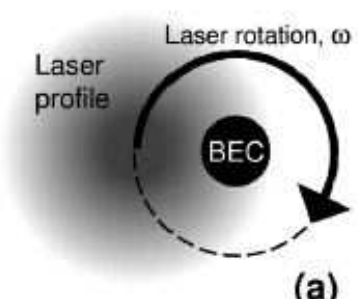

(a)

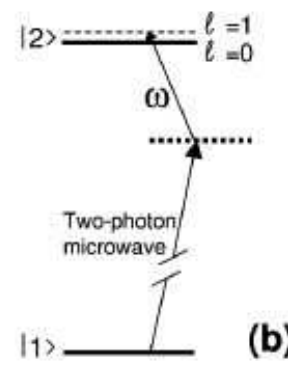

(b)
FIG. 3 (a) Schematic illustration of the technique used to create a vortex: An off-resonant laser provides a rotating force on the atoms across the condensate with a simultaneous microwave drive of detuning $\delta$. (b) Level diagram showing the microwave transition to the sublevels of the state $|2\rangle$. When the rotation frequency of the laser beam $\omega$ is close to the detuning $\delta$, the perturbation selectively modulates the transition from the $l=0$ state to the $l=1$ state. From Matthews et al. (1999b).

To create a quantized vortex, Williams and Holland
(1999) propose applying an external laser beam in addition to the external electromagnetic fields that produce the two-photon transition. This external laser beam in effect adds a rotating gradient to the coupling fields with a frequency $\omega$ (see Fig. 3). Such a process starts from the ground state $|1\rangle$ and yields the state $|2\rangle$ with one unit of angular momentum. Specifically, the theory predicts that the angular momentum of the state $|2\rangle$ oscillates and increases with time. Turning off the coupling at the appropriate time leaves the state $|2\rangle$ with a persistent singly quantized vortex because the associated order parameter for this state $|2\rangle$ now has $U(1)$ symmetry.

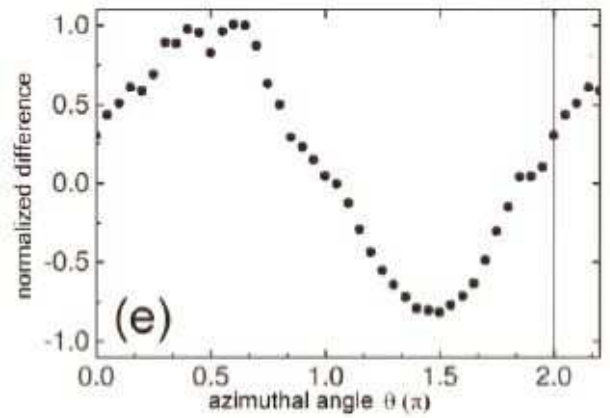

FIG. 4 Cosine of the relative phase around the vortex, showing the sinusoidal variation of the azimuthal angle. From Matthews et al. (1999b).

In the experiment (Matthews et al., 1999b), the vortex in state $|2\rangle$ surrounds a nonrotating core consisting of state $|1\rangle$. Furthermore, a $\pi$ pulse can interchange $|1\rangle$ and $|2\rangle$, so that the vortex can occur in either state. There is one important difference between the states $|1\rangle$ and $|2\rangle$, because the state $|2\rangle$ with $m_{F}=1$ is not maximally aligned. Consequently it can decay by spin relaxation with a measured lifetime $\sim 1 \mathrm{~s}$. As expected, a vortex in state $|1\rangle$ surrounding a core of state $|2\rangle$ is more stable because the state $|2\rangle$ core eventually decays, leaving a simple one-component vortex in state $|1\rangle$. The experiment can image either the filled core or the surrounding vortex, but these images by themselves do not demonstrate the presence of quantized circulation. Fortunately, the interference procedure described above allows a clear measurement of the relative phase between the two condensates at various positions, demonstrating the sinusoidal variation predicted for a vortex (see Fig. 4).

One very important advantage of this two-component experiment is the ability to follow the motion of the vortex core with nondestructive time-lapse images (Anderson et al., 2000). Figure 5 shows the uniform precession of the filled vortex core in state $|2\rangle$ around the trap center, determining the angular velocity of the circular motion. Note [Fig. [5(d)] the gradual shrinkage of the core radius as state $|2\rangle$ decays through spin-flip transitions. The experimenters can also remove the core component with a brief resonant laser pulse, leaving a one-component vortex whose empty core (of order $\xi$ ) is too small to image in the trap. To study the precession 


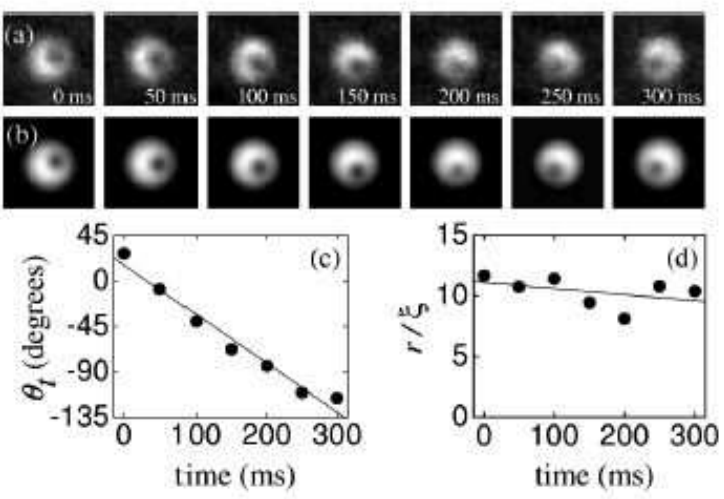

FIG. 5 (a) Successive images of a two-component condensate with a vortex in state $|1\rangle$ surrounding a core in state $|2\rangle$. (b) The image is then fit with a smooth Thomas-Fermi distribution. (c) The azimuthal angle of the vortex core plotted against the time, giving a precession frequency of $1.3(1) \mathrm{Hz}$. (d) The gradual decrease in the core radius $r$ (measured in units of the coherence length $\xi$ ) because of spin relaxation. From Anderson et al. (2000).

of such a one-component vortex, the experimenters first image the two-component vortex, then remove the core, wait a variable time and finally turn off the trap, imaging the expanded vortex core. In this way, they measure the precession rate of a one-component empty-core vortex, yielding values in reasonable agreement with theoretical predictions (Fetter and Svidzinsky, 2001). These data show no evidence that the vortex spirals outward, suggesting that thermal damping is negligible on the time scale of $\sim 1$ s.

\section{Vortex behavior in one-component BECs (ENS and subsequently others)}

Most other experimental methods of creating vortices in dilute BECs rely on the rotation of an anisotropic potential, and I briefly review the physics of such a system. Consider a classical incompressible fluid in a long cylinder with elliptical cross section $|x| \leq a$ and $|y| \leq b$. When the container rotates with angular velocity $\boldsymbol{\Omega}=\Omega \hat{\boldsymbol{z}}$, the moving walls (in the laboratory frame) exert forces on the fluid. As a result, the fluid executes a purely irrotational motion with velocity $\boldsymbol{v}_{\text {irr }}=\nabla \Phi_{\text {irr }}$ [see Eq. (2.36)], where the associated velocity potential $\Phi_{\text {irr }}=\Omega x y\left(a^{2}-b^{2}\right) /\left(a^{2}+b^{2}\right)$ is proportional to $x y$ [see pp. 86-88 of Lamb (1945)]. Remarkably, this irrotational velocity field induces a nonzero angular momentum per unit length $L_{\text {irr }}=L_{\mathrm{sb}}\left(a^{2}-b^{2}\right)^{2} /\left(a^{2}+b^{2}\right)^{2}$, where $L_{\mathrm{sb}}$ is the corresponding angular momentum per unit length for a fluid that executes solid-body rotation with $\boldsymbol{v}_{\mathrm{sb}}=\boldsymbol{\Omega} \times \boldsymbol{r}$. Note that this irrotational angular momentum occurs without quantized vortices. Such vortexfree angular momentum increases the critical angular velocity $\Omega_{c}$ for vortex formation in superfluid ${ }^{4}$ He Fetter,
1974), as DeConde and Packard (1975) verify by experiments on rotating elliptical and rectangular cylinders.

The same irrotational flow occurs in rotating anisotropic trapped BECs, where it appears in the phase of the condensate wave function $S=M \Phi_{\text {irr }} / \hbar$ [see Eq. [2.36] ] and leads to unexpected phenomena. For example, a sudden small rotation of the anisotropic trap potential induces an unusual oscillatory motion known as the "scissors mode" [see Secs. 14.3 and 14.4 of Pitaevskii and Stringari (2003) for a detailed description of this and related experiments].

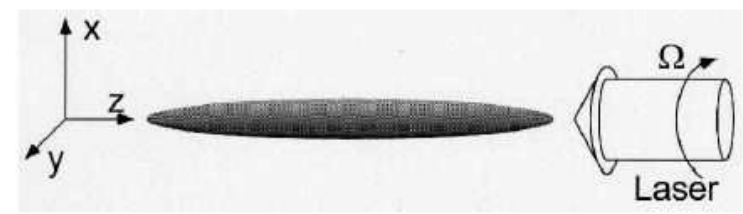

FIG. 6 A cigar-shaped condensate is confined by an axisymmetric magnetic trap and stirred by an off-center far-detuned laser beam. The laser beam propagates along the long axis and creates an anisotropic quadrupole potential that rotates at an angular velocity $\Omega$. From Madison et al. (2000b).

The experimental group at the Ecole Normale Supérieure (ENS) in Paris uses such a direct approach (see Fig. 6) to create one or more vortices in a one-component ${ }^{87} \mathrm{Rb}$ cigar-shaped condensate (Madison et al., 2000a). Starting with an axisymmetric condensate, they apply a toggled off-center stirring laser beam that slightly deforms the trap potential; the plane of deformation then rotates about the long axis with an applied angular velocity $\Omega /(2 \pi) \lesssim 200 \mathrm{~Hz}$. Physically, the laser beam acts on each polarizable atom in the BEC with a "dipole" force ${ }^{6}$ proportional to the gradient of the squared electric field of the laser [for the details of the stirring procedure, see (Madison et al., 2000b)]. This dipole-force mechanism is the basis for optical trapping of atoms, as discussed briefly in Sec. III.E in connection with optical lattices [for a more detailed account, see Sec. 4.2 of Pethick and Smith (2002) and Sec. 9.4 of Pitaevskii and Stringari (2003)].

Since the vortex core radius $\sim \xi \approx 0.2-0.4 \mu \mathrm{m}$ is too small for direct visual detection, they turn off the trap and allow the condensate (including the vortex core) to expand. The initial tight radial confinement means that this expansion is mostly in the radial direction, and the expanded condensate usually becomes disk-shaped. The resulting images of the expanded condensate clearly show the presence of one or a few vortices (see Fig. 7). These latter images are remarkably similar to those in Fig. 8 of vortices in rotating superfluid ${ }^{4} \mathrm{He}$ (Yarmchuk et al.,

\footnotetext{
6 Here, "dipole" means the induced atomic electric dipole; the actual deformation of the originally axisymmetric condensate usually has quadrupole symmetry.
} 
1979) obtained by trapping charged particles on the vortex cores [see Sec. 4.5 and Chap. 5 of Donnelly (1991)]. Hodby et al. (2002) (Oxford) observe similar vortex patterns with a rotating magnetic deformation of the trap potential. Their experimental arrangement allows them to explore the physics of vortex nucleation for relatively large quadrupole deformations.

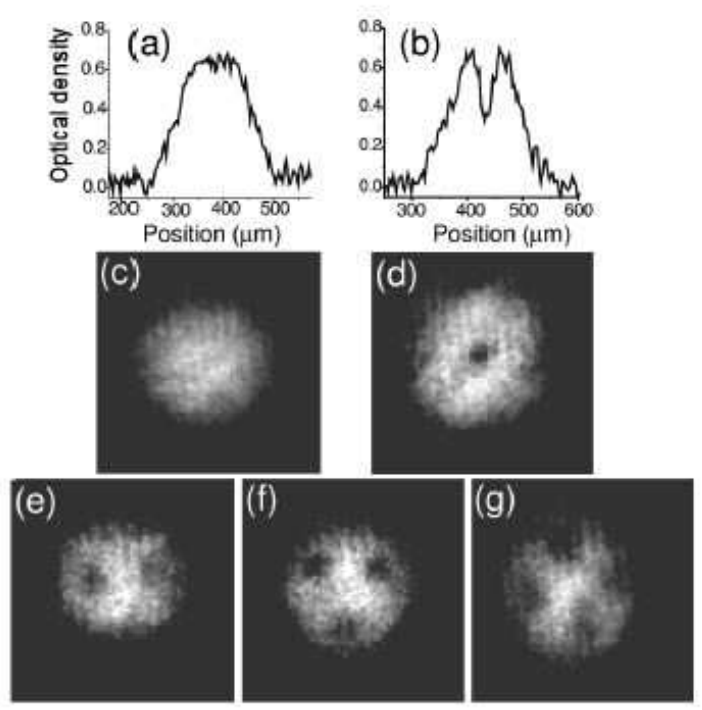

FIG. 7 Absorption images of a BEC stirred with a laser beam; in all images the condensate has about $10^{5}$ atoms, the temperature is below $80 \mathrm{nK}$ and the rotation rate $\Omega /(2 \pi)$ increases from $145 \mathrm{~Hz}$ for (a) and (c) to $168 \mathrm{~Hz}$ for (g). Images (a) and (b) show the optical thickness for images (c) and (d), with the clear appearance of the vortex core. Images (e), (f), and (g) show states with two, three, and four vortices. From Madison et al. (2000a).

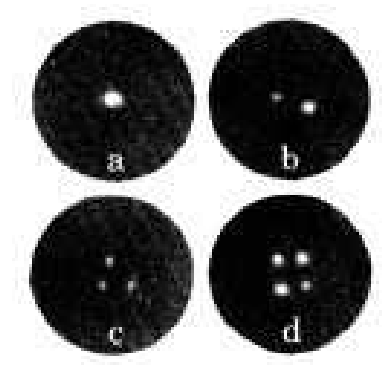

FIG. 8 Photographs of stable vortex configurations in superfluid ${ }^{4} \mathrm{He}$, with up to four vortices. Adapted from Yarmchuk et al. (1979).

Careful experimental studies (Chevy et al., 2000; Madison et al., 2001, 2000a , b) show that the critical angular velocity $\Omega_{c}$ for the appearance of the first vortex is $\approx 0.7 \omega_{\perp}$, which is typically much larger than that predicted in Eq. (3.10) for the relevant TF limit. In fact, a rotating axisymmetric condensate has a quadrupole instability at an angular velocity $\Omega=\omega_{\perp} / \sqrt{2}$, which agrees well with the measured value for the appear- ance of the first vortex. Sections 14.5 and 14.6 of Pitaevskii and Stringari (2003) discuss this intriguing behavior in detail.

\section{Normal modes of an axisymmetric condensate with one} central vortex

A static condensate without vortices has numerous low-lying small-amplitude normal modes characterized by coupled density and velocity perturbations. Many experimental studies have confirmed the various theoretical predictions in considerable detail [see pp. 178195 of Pethick and Smith (2002) and pp. 177-190 of Pitaevskii and Stringari (2003)]. A direct treatment of the hydrodynamic equations (Stringari, 1996) for a nonrotating axisymmetric condensate in the TF limit yields the frequency of quadrupole modes with $l=2, m=0$ and $l=2, m= \pm 2$. The last two modes with $m= \pm 2$ are degenerate and together produce an oscillating quadrupole distortion of the condensate in the plane perpendicular to the original axis of symmetry. In the absence of any perturbation that breaks time-reversal symmetry, the axes of the deformation remain fixed in space [see Figs. 9(a)].

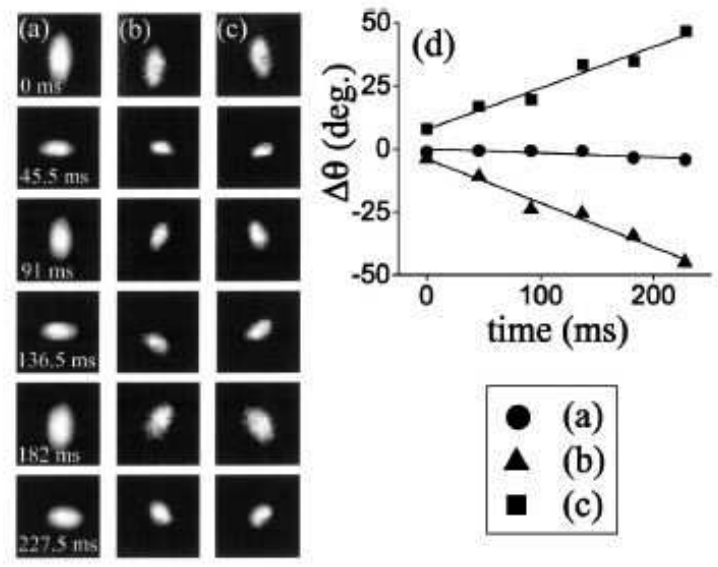

FIG. 9 Nondestructive images of quadrupole mode of an axisymmetric condensate. Condensate (a) is vortex-free, whereas condensates in (b) and (c) have straight vortices (with opposite sense of circulation) aligned perpendicular to the plane of the figure; (d) shows a linear fit to the precession angle. From Halian et al. (2001a).

When the condensate has a vortex, however, its circulating velocity breaks time-reversal symmetry and splits the previously degenerate states with $\pm m$ (Svidzinsky and Fetter, 1998; Zambelli and Stringari, 1998). This effect is analogous to the Zeeman splitting of magnetic sublevels. In the usual TF limit, the fractional splitting is small (of order $\left.|m| d_{\perp}^{2} / R_{\perp}^{2}\right)$. One dramatic consequence is that the quadrupole distortion now precesses slowly in the direction of the circulating vortex flow (Chevy et al., 2000; Haljan et al., 2001a), as seen in Figs. 9(b) and (c). 
Zambelli and Stringari (1998) note that the magnitude of the splitting provides a direct measure of the angular momentum of the condensate. The ENS group (Chevy et al., 2000) use this approach to detect the first appearance of a vortex in the condensate and to verify that its angular momentum per particle is $\sim \hbar$. It is remarkable that this macroscopic experiment with $N \sim 10^{6}$ atoms measures a single-particle fundamental constant; it demonstrates the coherence of the macroscopic wave function [see Sec. 4.7 of Leggett (2006) for a valuable perspective on this and related experiments]. Section 2.4 of Donnelly (1991) discusses similar earlier macroscopic experiments on rotating superfluid ${ }^{4} \mathrm{He}$.

The precession of the quadrupole mode provides a sensitive vortex detector (sometimes called "surface-wave spectroscopy") that complements the direct visibility of the vortex core. The JILA group (Halian et al., 2001a) use this method to track the large-amplitude tilting motion of a vortex in an axisymmetric condensate. As a tilted vortex rotates about the axis of symmetry, its empty core initially moves away from the line of sight. Periodically, however, the vortex returns to its original orientation, when the optical visibility of the core and the quadrupole precession both attain their initial values. The experiment indeed observes two recurrences, yielding good agreement with the predicted precession frequency (Svidzinsky and Fetter, 2000b).

\section{Bent vortex configurations}

When a disk-shaped condensate rotates faster than the critical velocity $\Omega_{m}$ for creation of a metastable vortex [see Eq. (3.11)], the initial equilibrium vortex is essentially straight. For condensates with larger aspect ratios $R_{z} / R_{\perp}$, however, the equilibrium vortex shape can be curved, depending on the angular velocity and the specific aspect ratio. Predictions of this phenomenon arise both from linearized stability analysis for a straight vortex (Feder et al., 2001; Svidzinsky and Fetter, 2000b) and from direct calculation of the state that minimizes the energy in the rotating frame (Aftalion and Rivière, 2001; García-Ripoll and Pérez-García, 2001a,b). In a long cigar-shaped condensate, for example, the initial vortex can reduce its total energy by forming a small symmetrical loop near the equator. As the angular velocity $\Omega$ increases, the vortex loop expands toward the axis of symmetry. When these vortex loops become large, ENS experiments (Rosenbusch et al., 2002) show that they can have both symmetric configurations (denoted "U") and antisymmetric configurations [denoted "(unfolded) N" although "S" or " $\int$ " might be more appropriate]. Modugno et al. (2003) give a nice physical picture of how curvature can lower the total energy of the vortex.

\section{Kelvin (axial) waves on a vortex}

A long straight classical vortex line in an incompressible fluid represents a dynamical equilibrium between the kinetic-energy density and the pressure, as follows from Bernoulli's equation. In an influential article, Thomson (1880) (subsequently, he became Lord Kelvin) studied the small oscillations of such a vortex; he found a characteristic long-wavelength $(|k| \xi \ll 1)$ dispersion relation

$$
\omega_{k} \approx \frac{\hbar k^{2}}{2 M} \ln \left(\frac{1}{|k| \xi}\right),
$$

where $k$ is the axial wave number, $\xi$ is the vortex core radius, and I assume a quantized circulation $\kappa=2 \pi \hbar / M$. For general $k$, these classical Kelvin modes are leftcircularly polarized helical waves with velocity potential $\propto \exp \left[i\left(k z-\phi-\omega_{k} t\right)\right] K_{1}(k r)$, where $(r, \phi, z)$ are cylindrical polar coordinates and $K_{1}(u)$ is a Bessel function of imaginary argument that vanishes for large $u$. Each element of the vortex core executes a circular orbit with a sense opposite to the circulating velocity of the vortex line. In a quantum-mechanical version, these modes have quantized angular momentum with $m=-1$. Note that this negative helicity $(m=-1)$ is independent of the direction of the wave propagation $( \pm k)$. The overall helical behavior reflects the gyroscopic character of the vortex line, and the physics thus differs significantly from that of a stretched string. ${ }^{7}$

Unfortunately, Kelvin's treatment is rather intricate, for he allowed the unperturbed axisymmetric flow to have an arbitrary radial dependence (including both solidbody rotation and irrotational vortex flow). For accessible discussions of these Kelvin modes, see Sec. 1.7 of Donnelly (1991), pp. 337-344 of Fetter (1969), pp. 138-139 of Fetter and Walecka (2006), Sec. 29 of Lifshitz and Pitaevskii (1980b) and Sec. III.D of Sonin (1987).

In a modern quantum-mechanical context, Pitaevskii (1961) used the Bogoliubov equations (2.41) and (2.42) to show that a singly quantized vortex in a dilute BEC also has this Kelvin mode with the same long-wavelength dispersion relation [Eq. (3.21)]. Although the existence of Kelvin waves are widely accepted, direct evidence for their presence in low-temperature superfluids is relatively meager. Section 6.1 of Donnelly (1991) and Sec. III.E of Sonin (1987) discuss the interpretation of relevant superfluid ${ }^{4} \mathrm{He}$ experiments in some detail.

The evidence is much more direct in dilute BECs. Bretin et al. (2003) report a clear signature of such quantized Kelvin modes. They drive the condensate with a near-resonant quadrupole excitation $(m= \pm 2)$ and observe preferential damping of the mode with $m=-2$. Recall that the Kelvin modes have quantized angular momentum $m=-1$ relative to the circulation around

\footnotetext{
${ }^{7}$ For a detailed comparison, see Fetter and Harvev (1971).
} 
the vortex, independent of the direction of $k$. Thus the authors suggest that this particular excited state with $m=-2$ can decay by the creation of two Kelvin-wave excitations, each with $m=-1$ and opposite momenta $\pm \hbar k$ along the axis determined by the conservation of energy. In contrast, the other quadrupole mode $m=+2$ has no such open channel for decay. A theoretical analysis of the line shape (Mizushima et al., 2003) confirms this interpretation. Furthermore, transverse images of the expanded condensate excited with the quadrupole mode $m=-2$ exhibit the expected periodic spatial structure associated with the Kelvin-mode deformation, whereas those excited with $m=+2$ have no periodic structure (Bretin et al., 2003).

\section{Other ways to create vortices}

As seen throughout Sec. III.D, experimentalists have devised many procedures that successfully create one or more vortices in a dilute BEC. Section III.D.1 discusses dynamical phase-imprinting in a two-component condensate, and Sec. III.D.2 considers the more common approach of rotating an anisotropic potential that spins up the condensate. A related technique (discussed below in Sec. IV.B in connection with vortex arrays) is to spin up the normal gas and then cool into the superfluid state (Halian et al., 2001b). One-dimensional solitons provide an additional unusual alternative, for they generally decay to form three-dimensional vortex rings (Anderson et al., 2001; Dutton et al., 2001).

a. transfer of orbital angular momentum from a laser A Gaussian laser beam can be decomposed into components with orbital angular momenta $m$ relative to the propagation vector (the Laguerre-Gaussian modes). Thus a laser beam can, in principle, create a vortex by coherently transferring orbital angular momentum to the condensate. In a recent experiment, Andersen et al. (2006) use a two-photon stimulated Raman process involving a counter-propagating linearly polarized Laguerre-Gaussian mode with $m=1$ [see, for example (Allen et al., 2003, 1999)] and a linearly polarized Gaussian mode with $m=0$ (the linear polarization ensures that "spin" angular momentum does not cause the observed effect). Some of the atoms in an initial single ${ }^{23} \mathrm{Na}$ condensate absorb a photon with one unit of angular momentum from the Laguerre-Gaussian beam and emit a photon to the Gaussian beam, acquiring both angular momentum and linear momentum. Consequently, these atoms form a new coherent moving condensate with a singly quantized vortex. After release from the trap, the resulting spatial separation between the new and the original condensates permits clear imaging of the vortex core.

Andersen et al. (2006) also use a sequence of such pulses to make vortices with higher quantized circula- tion; as noted below, however, such vortices are essentially unstable with respect to decay into singly quantized vortices. Recently, similar techniques create persistent quantized flow with one unit of circulation in a toroidal trap (Ryu et al., 2007); the $\sim 10$ s lifetime of this persistent flow arises from experimental factors and is not intrinsic.

b. merging of multiple BECs Another interesting experiment creates a vortex by merging three ${ }^{87} \mathrm{Rb}$ condensates in a single disk-shaped trap. Scherer et al. (2007) apply a repulsive (blue-detuned) laser beam shaped as a three-fold optical barrier, dividing the noncondensed gas into three non-overlapping regions (see Sec. III.E for the effect of external laser beams). As the gas cools below $T_{c}$, three independent and uncorrelated BECs form. The optical barrier is then slowly removed, and the three BECs merge. After a variable delay time, they remove the trap and image the expanded condensate. Depending on the initial relative phases of the three condensates, a net circulating current can form, producing a clearly visible vortex core in the expanded image. For random phase differences, a vortex should appear with probability 0.25 , in reasonable agreement with the experimental observations for repeated trials.

c. imposition of topological phase As a final topic in this discussion of forming a single vortex, I consider the creation of a doubly quantized vortex through imposition of topological phase (Leanhardt et al., 2002). The experiment follows suggestions by Isoshima et al. (2000) and Ogawa et al. (2002). It relies on a special form of magnetic confinement known as the Ioffe-Pritchard trap [for a detailed description, see Sec. 4.1.3 of Pethick and Smith (2002)]. Here, the important feature is that the $x$ and $y$ components of the magnetic field have a quadrupole form, whereas the $z$ component can be taken as uniform.

This MIT experiment studies ${ }^{23} \mathrm{Na}$ in the lower hyperfine state $\left|F=1, m_{F}=-1\right\rangle$. Initially, the $z$ component of magnetic field is large and positive, which aligns the hyperfine spin $\boldsymbol{F}$ vector with the direction of $\boldsymbol{B} \approx B_{z} \hat{\boldsymbol{z}}$. Thus this spin texture is uniform and oriented, with variable number density induced by the nonuniform trap potential. The experiment then slowly reverses the $z$ component of magnetic field, and the hyperfine spin vector $\boldsymbol{F}$ adiabatically follows the local magnetic field. In particular, when $B_{z}$ vanishes, the hyperfine spin vector $\boldsymbol{F}$ takes the (nonuniform) quadrupole form of the remaining $x$ and $y$ components of $\boldsymbol{B}$. As $B_{z}$ then becomes negative, the topological winding of the spin texture continues adiabatically, leading to a nontrivial final texture. Remarkably, at the end of the $B_{z}$ inversion, a detailed analysis shows that the local spin texture has an additional factor $e^{-i 2 m_{F} \phi}$, where $m_{F}=-1$. Consequently, this final state represents a doubly quantized vortex with quantum number two. Leanhardt et al. (2002) use surface-wave spec- 
troscopy (precession of the quadrupole oscillation mode, see Sec. III.D.3) to verify that the vortex indeed has angular momentum $\approx 2 \hbar$ per particle.

The energy of a vortex arises principally from the total kinetic energy $\int d V \frac{1}{2} M v^{2}|\Psi|^{2}$, which is proportional to the square of the circulation. In a multiply connected geometry like an annulus, such flow represents a macroscopic quantized circulation, and the presence of the inner wall ensures that the hydrodynamic flow generally remains stable (a persistent current) as long as the temperature is well below $T_{c}$. The situation is very different for a free-standing multiply quantized vortex, which should be unstable with respect to splitting into the appropriate number of singly quantized vortices. As mentioned at the end of Sec. II.C, $\mathrm{Pu}$ et al. (1999) study the Bogoliubov equations for a doubly quantized vortex and find imaginary eigenvalues that imply a dynamical instability [see also (Castin and Dum, 1999)]. Shin et al. (2004) investigate the stability of these doubly quantized vortices, with convincing images of the relatively large initial vortex core decaying into two smaller cores in $\approx 75 \mathrm{~ms}$. Möttönen et al. (2003) provide a theoretical analysis of the MIT experiments, emphasizing the unstable eigenvalues of the Bogoliubov equations.

An alternative picture of the same topological transformation (Leanhardt et al., 2002; Ogawa et al., 2002) relies on the idea of Berry (1984) phase that arises from an adiabatic alteration of the physical system (in this case, the overall direction of the magnetic field). In the context of quantum mechanics, many recent textbooks provide a careful description of the Berry phase, for example, Sec. 10.3 of Abers (2004), Sec. 10.2 of Griffiths (2005), and pp. 592-607 of Shankar (1994).

\section{E. Laser trapping and optical lattices}

The response of an atom to electromagnetic radiation is an old subject [see, for example, Allen and Eberly (1987) and Cohen-Tannoudji et al. (1998)], yet it has many important applications to the interaction between ultracold dilute atoms and laser beams. To understand the basic physics, place an electric dipole with moment $\boldsymbol{p}$ in an external electric field $\boldsymbol{E}$. Standard electrostatics yields the interaction energy $U_{\mathrm{int}}=-\boldsymbol{p} \cdot \boldsymbol{E}$, where the minus sign means that the lowest-energy configuration has the dipole moment oriented along $\boldsymbol{E}$.

This expression holds for a fixed dipole moment $\boldsymbol{p}$, but an atom typically acquires its electric dipole moment as a result of induced polarization. In such a case, the dipole moment is proportional to the applied field, with $\boldsymbol{p}=\alpha \epsilon_{0} \boldsymbol{E}$ in SI units, where $\epsilon_{0} \approx 8.85 \times 10^{-12}$ farad $/ \mathrm{m}$ is the permittivity of empty space and $\alpha$ is the atomic polarizability. As a very simple classical model, a grounded conducting sphere of radius $b$ has a polarizability $\alpha=4 \pi b^{3}$ (three times its volume), and this picture provides a useful order-of-magnitude estimate for atomic polarizabilities. The total interaction energy of the atom with the external field then builds up from $\boldsymbol{E}_{i}=\mathbf{0}$, and integration yields the final expression

$$
U_{\text {int }}=-\frac{1}{2} \alpha \epsilon_{0} E^{2} .
$$

If the electric field $\boldsymbol{E}(\boldsymbol{r})$ is static and nonuniform, then $U_{\text {int }}(\boldsymbol{r})=-\frac{1}{2} \alpha \epsilon_{0} E^{2}(\boldsymbol{r})$ is also nonuniform. Thus an atom experiences a force that seeks to minimize the interaction energy, placing the atom at the maximum of $E^{2}$ because the static polarizability is positive. This result also holds for low frequencies, but the situation eventually changes because of induced transitions among atomic energy levels. The Drude model of a bound electron with resonant frequency $\omega_{0}$ and damping time $\tau$ subject to an oscillatory driving field yields the frequency-dependent polarizability

$$
\alpha(\omega)=\frac{\alpha(0) \omega_{0}^{2}}{\omega_{0}^{2}-\omega^{2}-i \omega / \tau},
$$

where $\alpha(0)$ is the (positive) static polarizability. Note that the real part of $\alpha(\omega)$ changes from positive to negative when the applied frequency $\omega$ exceeds the resonant frequency $\omega_{0}$. The combined relation $U_{\text {int }}(\omega)$ from Eqs. (3.22) and (3.23) is sometimes called the ac Stark effect.

These ideas apply directly to the optical trapping of atoms by a focused laser. If the frequency $\omega$ of the laser is less than that of the atomic resonance ("red detuning") with $\operatorname{Re} \alpha(\omega)>0$, then Eqs. (3.22) and (3.23) indicate that the atoms preferentially move toward the squared-field maxima, whereas if the laser frequency is higher than the resonant frequency ("blue detuning") with $\operatorname{Re} \alpha(\omega)<0$, then the atoms move toward the squared-field minima. In practice, it is necessary to use far-off-resonance lasers to avoid dissipation. For the alkali-metal atoms of interest here $(\mathrm{Li}, \mathrm{Na}, \mathrm{K}, \mathrm{Rb}$, $\mathrm{Cs}$ ), the lowest $s$ to $p$ transition of the valence electron predominates, as in the familiar yellow emission spectrum of Na. Stamper-Kurn et al. (1998) successfully trap a pre-existing ${ }^{23} \mathrm{Na} \mathrm{BEC}$ with a focused infrared laser beam. Unlike magnetic traps, which confine only certain hyperfine states, these optical traps confine all such states. This experimental technique allows the study of "spinor" condensates (see Sec. VII.B.3) that involve coupled BECs, one for each hyperfine component of a given $\boldsymbol{F}$ multiplet (Ho, 1998; Ohmi and Machida, 1998).

A more subtle example is the formation of an optical standing wave to trap atoms in a periodic array. Consider a laser beam propagating along the $z$ axis with electric field $\boldsymbol{E}(z, t)=\boldsymbol{E}_{0} \cos (k z-\omega t)$. If the laser beam reflects normally off a mirror at $z=0$, the resulting standing wave $\boldsymbol{E}_{0}[\cos (k z-\omega t)-\cos (k z+\omega t)]=$ $2 \boldsymbol{E}_{0} \sin k z \sin \omega t$ yields the corresponding squared electric field $|E(z, t)|^{2}=4\left|E_{0}\right|^{2} \sin ^{2} k z \sin ^{2} \omega t$. This periodic lattice has a spatial period $\frac{1}{2} \lambda$, where $\lambda=2 \pi / k$ is the wavelength of the original laser beam. For either sign of detuning, the atoms assume a spatially periodic configuration in this optical lattice, as emphasized by Jaksch et al. 
(1998). This seminal idea has wide applications in the study of ultracold dilute gases, as discussed, for example, in Sec. 9.4 and Chap. 16 of Pitaevskii and Stringari (2003) and Sec. IV of Bloch et al. (2007). The strength of the laser field can be controlled externally, so that the height of the barrier for tunneling between adjacent layers can vary from small to large. This possibility leads to remarkable experiments that demonstrate the transition from superfluid to insulating behavior in three-dimensional optical lattices (Greiner et al., 2002). In the present context of one-dimensional lattices, Anderson and Kasevich (1998) are the first to demonstrate such atom trapping. Their vertical lattice experiences a gravitational potential difference $M g \lambda / 2$ between adjacent layers, with associated interlayer tunneling at a measured ac Josephson frequency $\omega_{J} \approx$ $M g \lambda /(2 \hbar)$ (Josephson, 1962).

A particularly interesting situation arises when a rotating condensate is subsequently confined in a onedimensional optical lattice oriented along the rotation axis. Consider an axisymmetric condensate with a single vortex on the axis of symmetry. Then adiabatically turn on an applied standing-wave laser field. If the laser beams propagate along the symmetry axis, the optical lattice slices the rotating condensate into many circular disks. The resulting periodic atomic array forms a set of effectively two-dimensional condensates that are coupled by tunneling between adjacent layers. Each twodimensional condensate contains a segment of the previously continuous vortex line. Thus it becomes a single pancake vortex, and the position of each vortex center can fluctuate more-or-less independently, depending on the strength of the laser field and the height of resulting potential barriers. Martikainen and Stoof (2003) use a variational trial function to study the resulting axial modes in this discrete system, which are the analog of the Kelvin modes studied in Sec. III.D.5. At present, no direct experimental evidence for such behavior exists.

\section{F. Berezinskii-Kosterlitz-Thouless transition in dilute BECs}

Another remarkable application of an optical lattice is the study of the equilibrium state of a twodimensional dilute Bose-Einstein gas. Although such a uniform two-dimensional system cannot have a true condensate with long-range order (Hohenberg, 1967; Mermin and Wagner, 1966), nevertheless Berezinskii (1971) and Kosterlitz and Thouless (1973) predict that it can indeed become superfluid below a certain critical temperature $T_{B K T}$. Above this temperature, free single vortices can form easily. The motion of such vortices disrupts any organized long-range phase coherence (Leggett, 2001) and hence destroys the associated superfluid flow. Below $T_{B K T}$, in contrast, free vortices do not occur, although bound pairs of a vortex and an antivortex do exist [in the present context of dilute ultracold gases, Sec. 17.5 of Pitaevskii and Stringari (2003) discusses this behav- ior in more detail]. As the temperature increases, so does the size of the bound pairs, and they eventually become unbound at $T_{B K T}$, producing free vortices. Experiments with thin superfluid ${ }^{4} \mathrm{He}$ films (Bishop and Reppy, 1978) and with Josephson-coupled superconducting arrays (Resnick et al., 1981) have both confirmed these predictions in considerable detail.

In a recent experiment at ENS (Paris), Hadzibabic et al. (2006) apply a standing-wave laser field perpendicular to the symmetry axis of a cigar-shaped condensate, splitting the atoms into two essentially independent thin pancake strips with lateral dimensions $\approx 120 \mu \mathrm{m} \times 10 \mu \mathrm{m}$. See Fig. 6 for the geometry (although there is no rotating laser beam in the present case): the laser beams propagate in the $x$ direction and the pancakes are parallel to the $y z$ plane. After these two isolated Bose-Einstein gases reach thermal equilibrium, the confining traps are turned off. Both pancakes then expand primarily in the $x$ direction perpendicular to their planes and overlap spatially.

Soon after the first observations of BECs in dilute trapped gases, the MIT group (Andrews et al., 1997) cut a cigar-shaped condensate in half with a blue-detuned laser, creating two separate three-dimensional condensates. When the trap is turned off, these two condensates overlap and produce clear straight interference fringes. Similarly, the ENS experiment here looks for and finds interference fringes between the two pancakes. At low temperature [see Fig 10(a)], the fringes are indeed straight, indicating constant relative phase. As the temperature increases, however, the fringes become wavy [Fig 10(b)]; furthermore, as seen in Fig 10)(c), they sometimes contain one or more dislocations, which indicates the presence of free vortices in one or both pancakes (Simula and Blakie, 2006; Stock et al., 2005b). They repeat the experiment at various temperatures and use the presence of free vortices to determine $T_{B K T}$. The resulting value agrees well with the theoretical predictions of Berezinskii (1971) and Kosterlitz and Thouless (1973), although the finite size of the present samples yields a crossover instead of a sharp transition.

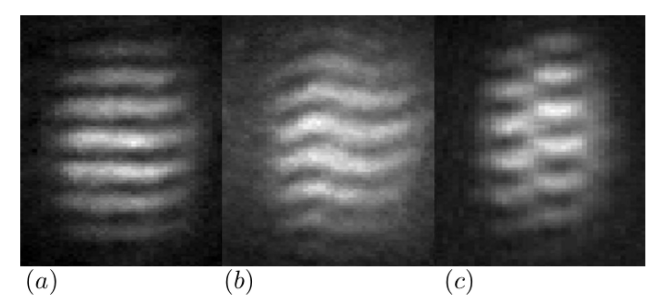

FIG. 10 Interference fringes between two pancake condensates (a) at low temperature, (b) at high temperature showing wavy interference fringes without vortices, (c) fringe dislocation indicates the presence of a vortex in one or both condensates. From Krüger (2007).

Schweikhard et al. (2007) report an intriguing study of vortex formation in a two-dimensional array of Josephson-coupled BECs. They start at $T$ below $T_{c}$ with 
a disk-shaped condensate along with a residual normal cloud and then apply a hexagonal optical lattice, transforming the system into an array of coupled BECs that act like Josephson junctions. Each condensate has a single effective phase, and the coupling energy between adjacent condensates has the form $J(1-\cos \Delta \phi)$ where $\Delta \phi$ is their relative phase. Equivalently, the tunneling current between any pair of condensates is proportional to $J \sin \Delta \phi$ [for a careful discussion, see Josephson (1962), Leggett (2001), and Chap. 6 of Tinkham (1996)]. Varying the amplitude of the optical lattice through the laser intensity tunes the coupling parameter $J$.

The experiment starts by inferring the temperature $T$ from a nondestructive image and then ramps down the optical lattice, allowing the adjacent BECs to merge. As in Scherer et al. (2007), discussed in Sec. III.D.6, vortices can form depending on the relative phases, appearing as holes in the expanded gas cloud after the trap is turned off. For a given temperature $T$, Schweikhard et al. (2007) measure the vortex density $\mathcal{D}$ near the center of the cloud as a function of the Josephson coupling parameter $J$. For two different temperatures, a plot $\mathcal{D}$ as a function of $J / T$ shows nearly universal behavior: $\mathcal{D}$ is negligibly small for $J / T \gg 1$ (low temperature), but it rises rapidly and saturates for $J / T \ll 1$ (high temperature), as expected for the BKT vortex unbinding crossover.

\section{VORTEX ARRAYS IN MEAN-FIELD THOMAS-FERMI (TF) REGIME}

A rapidly rotating condensate has a dense array of vortices, with a uniform areal density (Feynman, 1955)

$$
n_{v}=\frac{M \Omega}{\pi \hbar}
$$

as follows from these arguments:

(1) Consider a steady classical incompressible flow with velocity $\boldsymbol{v}(\boldsymbol{r})$ in a rigid container that rotates with angular velocity $\boldsymbol{\Omega}$. If the fluid has number density $n(\boldsymbol{r})$, then the relevant part of the energy in the rotating frame is

$$
E^{\prime}=\int d V\left(\frac{1}{2} M v^{2}-M \boldsymbol{\Omega} \cdot \boldsymbol{r} \times \boldsymbol{v}\right) n .
$$

This result is equivalent to

$$
E^{\prime}=\frac{1}{2} M \int d V(\boldsymbol{v}-\boldsymbol{\Omega} \times \boldsymbol{r})^{2} n-E_{\mathrm{sb}},
$$

where $E_{\mathrm{sb}}=\frac{1}{2} M \int d V|\boldsymbol{\Omega} \times \boldsymbol{r}|^{2} n=\frac{1}{2} I_{\mathrm{sb}} \Omega^{2}$ is the rotational energy for solid-body rotation with the corresponding moment of inertia $I_{\mathrm{sb}}$. The first term of Eq. (4.3) is non-negative, and hence the absolute minimum of $E^{\prime}$ occurs when $\boldsymbol{v}=\boldsymbol{\Omega} \times \boldsymbol{r}$, namely solid-body rotation $\boldsymbol{v}_{\mathrm{sb}}=\boldsymbol{\Omega} \times \boldsymbol{r}$; in addition, the corresponding minimum value of $E_{\min }^{\prime}$ has the classical value $-E_{\mathrm{sb}}$. Since $\boldsymbol{\nabla} \times \boldsymbol{v}_{\mathrm{sb}}=2 \boldsymbol{\Omega}$, the irrotational superflow cannot assume such a solid-body rotation, but it seeks to mimic this flow through the nucleation of quantized vortices.
The angular momentum $L_{z}=\langle\boldsymbol{r} \times \boldsymbol{p}\rangle$ follows from the thermodynamic relation

$$
L_{z}=-\left(\partial E^{\prime} / \partial \Omega\right)_{N}
$$

[the Hellmann-Feynman theorem gives the same result. See, for example, pp. 1192-1193 of Cohen-Tannoudii et al. (1977); for a brief history of this theorem, see p. 1 of Brown (2000)]. Since the actual $E^{\prime}$ exceeds the classical value $-\frac{1}{2} I_{\mathrm{sb}} \Omega^{2}$ by a positive correction that depends on $\Omega$, the equilibrium value of the angular momentum necessarily differs from the classical value $I_{\mathrm{sb}} \Omega$. Evidently, this system exhibits a nonclassical moment of inertia [see Sec. 2.5 of Leggett (2006) for a discussion of these quantum-mechanical effects]. In addition, it also has transverse shear modes (the Tkachenko modes, see Sec. IV.B.4), and it "melts" for sufficiently large angular velocities to form a "liquid" state (see Sec. VI). Whether this system should be considered a "supersolid" in the conventional sense [see, for example, Sec. 8.3 of Leggett (2006)] merits additional study.

(2) For a superfluid, Eq. (3.3) shows that a singly quantized vortex at a position $\boldsymbol{r}_{0}$ has a localized vorticity $\boldsymbol{\nabla} \times \boldsymbol{v}=(2 \pi \hbar / M) \delta^{(2)}\left(\boldsymbol{r}-\boldsymbol{r}_{0}\right) \hat{\boldsymbol{z}}$. In a rotating superfluid, the vortices will be uniformly distributed to approximate the uniform classical vorticity. Take a contour $\mathcal{C}$ containing $\mathcal{N}_{v}$ such vortices in an area $\mathcal{A}$; the total circulation around $\mathcal{C}$ is $\Gamma_{\mathcal{C}}=(2 \pi \hbar / M) \mathcal{N}_{v}$. Stokes's theorem shows that the corresponding classical value would be $\Gamma_{\mathcal{C}}^{\mathrm{cl}}=2 \Omega \mathcal{A}$, and comparison immediately yields the mean areal vortex density $n_{v}=\mathcal{N}_{v} / \mathcal{A}=M \Omega /(\pi \hbar)$. The inverse $n_{v}^{-1}$ is the area per vortex $\pi \hbar /(M \Omega) \equiv \pi l^{2}$, which defines the radius

$$
l=\sqrt{\frac{\hbar}{M \Omega}}
$$

of an equivalent circular cell. Thus the intervortex separation is $\approx 2 l$. Note that $l$ decreases with increasing $\Omega$ (it is also the usual oscillator length for a frequency $\Omega$ ).

\section{A. Physics of BEC in axisymmetric harmonic traps for rapid rotation}

The Feynman relation (4.1) shows that the number of vortices increases linearly with $\Omega$ assuming that the geometry remains fixed. Although this picture indeed applies to superfluid ${ }^{4} \mathrm{He}$ in a rotating bucket, the situation is quite different for a dilute gas in a harmonic trap, because the centrifugal forces expand the condensate radially (number conservation means that the condensate also shrinks along the axis of rotation). As a result, the total number of vortices in a given condensate

$$
\mathcal{N}_{v}(\Omega) \approx n_{v} \pi R_{\perp}^{2}(\Omega)=M \Omega R_{\perp}^{2}(\Omega) / \hbar=R_{\perp}^{2}(\Omega) / l^{2}
$$

increases faster than linearly with $\Omega$. 
To quantify the dependence of $R_{\perp}$ on $\Omega$, it is convenient to focus on the TF limit of a large condensate, when the spatial variation of the condensate density is negligible compared to the other terms in the energy functional. Thus $\boldsymbol{p} \Psi=-i \hbar \boldsymbol{\nabla} \Psi=(\hbar \nabla S) \Psi-i \hbar e^{i S} \boldsymbol{\nabla}|\Psi| \approx M \boldsymbol{v} \Psi$, and the GP energy functional in the rotating frame Eq. (3.6) simplifies to

$$
\begin{gathered}
E^{\prime}[\Psi] \approx \int d V\left[\left(\frac{1}{2} M v^{2}-M \boldsymbol{\Omega} \cdot \boldsymbol{r} \times \boldsymbol{v}+V_{\mathrm{tr}}\right)|\Psi|^{2}\right. \\
\left.+\frac{1}{2} g|\Psi|^{4}\right] .
\end{gathered}
$$

Like the TF limit $E_{\mathrm{TF}}[\Psi]$ in Eq. (2.24) for a stationary condensate, this equation depends only of $|\Psi|^{2}$ and $|\Psi|^{4}$, but it now includes the hydrodynamic flow through $\boldsymbol{v}$.

In the typical case of rotation about $\hat{\boldsymbol{z}}$, simple manipulations yield the equivalent expression

$$
\begin{aligned}
E^{\prime}[\Psi] & \approx \int d V \frac{1}{2} M\left(\boldsymbol{v}-\boldsymbol{v}_{\mathrm{sb}}\right)^{2}|\Psi|^{2} \\
& +\int d V\left[\left(V_{\mathrm{tr}}-\frac{1}{2} \Omega^{2} r^{2}\right)|\Psi|^{2}+\frac{1}{2} g|\Psi|^{4}\right] \\
& \equiv E_{v}^{\prime}+E_{\mathrm{TF}}^{\prime},
\end{aligned}
$$

where $E_{v}^{\prime}$ and $E_{\mathrm{TF}}^{\prime}$ denote the contributions in the first and second lines. For a large condensate with many vortices, the actual superfluid velocity $\boldsymbol{v}$ closely approximates the classical solid-body value $\boldsymbol{v}_{\mathrm{sb}}$, so that $E_{v}^{\prime}$ [the term containing $\left(\boldsymbol{v}-\boldsymbol{v}_{\mathrm{sb}}\right)^{2}$ ] vanishes to leading order. The remaining terms $E_{\mathrm{TF}}^{\prime}(\Omega)$ have exactly the same form as $E_{\mathrm{TF}}$ in Eq. (2.24), but the radial part of the confining potential is altered to $\frac{1}{2} M\left(\omega_{\perp}^{2}-\Omega^{2}\right) r^{2}$. Variation with respect to $|\Psi|^{2}$ gives an equation of the same form as Eq. (2.25), but with the reduced radial confining potential. Thus the TF density again has the form of Eq. (2.27), but with modified TF condensate radii that depend on $\Omega$. The normalization condition in three dimensions shows that

$$
\mu_{\mathrm{TF}}(\Omega)=\mu_{\mathrm{TF}}(0)\left(1-\Omega^{2} / \omega_{\perp}^{2}\right)^{2 / 5},
$$

so that the chemical potential decreases continuously and formally vanishes for $\Omega \rightarrow \omega_{\perp}$, as does the central density $\mu_{\mathrm{TF}}(\Omega) / g$ (because of the reduced radial confinement). A combination of these results leads to the specific $\Omega$ dependence of the condensate dimensions (Butts and Rokhsar, 1999; Fetter, 2001; Raman et al., 2001)

$$
\frac{R_{\perp}(\Omega)}{R_{\perp}(0)}=\left(1-\frac{\Omega^{2}}{\omega_{\perp}^{2}}\right)^{-3 / 10}, \frac{R_{z}(\Omega)}{R_{z}(0)}=\left(1-\frac{\Omega^{2}}{\omega_{\perp}^{2}}\right)^{1 / 5}
$$

As anticipated, the external rotation expands the condensate radially and shrinks it axially, approaching a twodimensional configuration. The corresponding aspect ratio

$$
\frac{R_{z}(\Omega)}{R_{\perp}(\Omega)}=\frac{\sqrt{\omega_{\perp}^{2}-\Omega^{2}}}{\omega_{z}}
$$

provides a convenient diagnostic tool to infer the actual angular velocity of the rotating condensate (Halian et al.
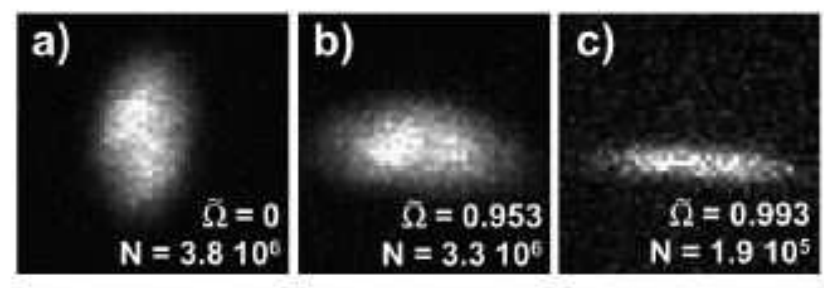

FIG. 11 Side view of BECs in a trap. (a) Static BEC, with aspect ratio $R_{z} / R_{\perp}=1.57$. Dramatically reduced aspect ratio is evident for (b) $\Omega / \omega_{\perp}=0.953$ and (c) $\Omega / \omega_{\perp}=0.993$. From Schweikhard et al. (2004).

2001b; Raman et al., 2001; Schweikhard et al., 2004), as seen in Fig. [11.

The two terms in Eq. (4.8) have quite different magnitudes in the mean-field TF regime. The dominant contribution $E_{\mathrm{TF}}^{\prime}(\Omega)$ is the TF energy of the rotating condensate with the modified squared radial trap frequency $\omega_{\perp}^{2} \rightarrow \omega_{\perp}^{2}-\Omega^{2}$. This quantity follows directly from Eqs. (2.30) and (4.9)

$$
E_{\mathrm{TF}}^{\prime}(\Omega)=\frac{5}{7} \mu_{\mathrm{TF}}(\Omega) N \propto\left(1-\Omega^{2} / \omega_{\perp}^{2}\right)^{2 / 5} .
$$

The thermodynamic relation in Eq. (4.4) yields the corresponding TF angular momentum of the rotating condensate, and some algebra gives the simple expression

$$
L_{\mathrm{TF}}=\frac{2}{7} M N R_{\perp}^{2}(\Omega) \Omega=I_{\mathrm{sb}}(\Omega) \Omega,
$$

where $I_{\mathrm{sb}}(\Omega)=\frac{2}{7} M N R_{\perp}^{2}(\Omega)$ is the solid-body moment of inertia for the deformed rotating condensate (this expected result follows from a separate calculation of $\left.\left\langle r^{2}\right\rangle_{\mathrm{TF}}\right)$.

The remaining term $E_{v}^{\prime}$ in Eq. (4.8) gives a small nonclassical contribution to the angular momentum of the rotating condensate. If the volume integral is approximated as a sum of integrals over circular cylindrical cells of radius $l$ centered at each vortex, a straightforward analysis yields

$$
E_{v}^{\prime}(\Omega) \approx N \hbar \Omega \ln (l / \xi)
$$

With logarithmic accuracy (ignoring the $\Omega$ dependence inside the logarithm), the corresponding nonclassical angular momentum then becomes

$$
L_{v}^{\prime}=-\frac{\partial E_{v}^{\prime}}{\partial \Omega} \approx-N \hbar \ln \left(\frac{l}{\xi}\right)
$$

Thus the total angular momentum of the rotating condensate indeed displays nonclassical behavior [see Secs. 2.5 and 8.3 of Leggett (2006)]

$$
L_{z} \approx I_{\mathrm{sb}}(\Omega) \Omega-N \hbar \ln (l / \xi) .
$$

Note that the correction reflects the inability of the superfluid to rotate like a solid body. It arises directly from the quantization of circulation and would vanish in the classical limit $(\hbar \rightarrow 0)$ when $\kappa \rightarrow 0$ and $n_{v}=2 \Omega / \kappa \rightarrow \infty$. The correction $L_{v}^{\prime}$ also becomes small in the limit of a dense vortex array $(l \rightarrow \xi)$, as discussed in Sec. V.B.1. 


\section{B. Experimental and theoretical studies of vortex lattices in the TF regime}

The first images of one or a few vortices (Sec. III.D) rapidly led to the creation of large vortex arrays. Initially, the MIT group (Abo-Shaeer et al., 2001) use a rotating deformation (like the ENS technique - see Fig. 6) to obtain remarkable images of triangular vortex lattices that are strikingly uniform, even near the outer edge of the condensate. These arrays closely resemble the classic triangular flux-line lattice in type-II superconductors predicted by Abrikosov (1957) and the triangular vortex lattice in rotating neutral incompressible superfluids predicted by Tkachenko (1966a).

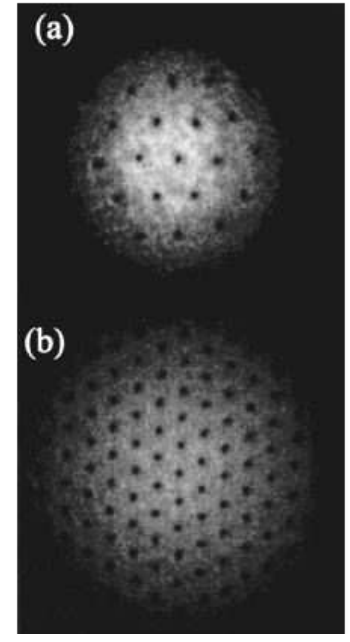

FIG. 12 Images of expanded ${ }^{87} \mathrm{Rb}$ condensates showing (a) small vortex array for slow rotation and (b) large vortex array for rapid rotation. Note the highly regular triangular form. Adapted from Coddington et al. (2004).

The JILA group (Engels et al., 2003; Haljan et al., 2001b) take a quite different approach to imparting angular momentum to the condensate. They start by spinning-up a normal cloud above $T_{c}$ with an elliptically deformed rotating disk-shaped trap. To cool into the BEC regime, they adiabatically alter their trap to cigarshaped and then evaporate normal atoms near the ends of the axis of symmetry (these atoms have small angular momentum). This process yields a rotating condensate with relatively large angular momentum per particle and good triangular arrays, as seen in Fig. 12, These rapidly rotating BECs facilitate a series of detailed experiments on the properties of the vortex lattice.

\section{Collective modes of rotating condensates}

The study of collective modes of a static condensate provides one of the crucial tests of the time-dependent GP equation, as discussed in Sec. IV of Dalfovo et al. (1999) and Chap. 12 of Pitaevskii and Stringari
(2003). One particularly effective theoretical approach (Stringari, 1996) linearizes the hydrodynamic equations (2.38) and (2.39) in the small perturbations $\delta n$ and $\delta \boldsymbol{v}=\boldsymbol{\nabla} \delta \Phi$. Since this formalism explicitly assumes irrotational flow, it fails for a rapidly rotating $\mathrm{TF}$ condensate with a large vortex lattice, where the average superfluid velocity has uniform vorticity $\boldsymbol{\nabla} \times \boldsymbol{v} \approx 2 \boldsymbol{\Omega}$.

Cozzini and Stringari (2003) propose a simple generalization, based on the Euler equation of nonviscous hydrodynamics, retaining the full convective term $\partial \boldsymbol{v} / \partial t+$ $(\boldsymbol{v} \cdot \boldsymbol{\nabla}) \boldsymbol{v}$. A familiar vector identity yields the equivalent form of the Euler equation in the laboratory frame

$$
M \frac{\partial \boldsymbol{v}}{\partial t}+\nabla\left(\frac{1}{2} M v^{2}+V_{\mathrm{tr}}+g n\right)=M \boldsymbol{v} \times(\boldsymbol{\nabla} \times \boldsymbol{v})
$$

which includes both the trap potential $V_{\mathrm{tr}}$ and the Hartree potential $g n$, but omits the quantum kinetic energy from Eq. (2.39), as appropriate in the TF limit. For a nonrotating irrotational condensate, $\boldsymbol{\nabla} \times \boldsymbol{v}$ vanishes, and Eq. (4.17) then omits the right-hand side, reproducing the gradient of Eq. (2.39) in the TF limit. In contrast, a rotating $\mathrm{TF}$ condensate has many vortices, with $\boldsymbol{\nabla} \times \boldsymbol{v} \approx 2 \boldsymbol{\Omega}$. Thus Cozzini and Stringari (2003) replace the right-hand side of Eq. (4.17) with $2 M \boldsymbol{v} \times \boldsymbol{\Omega}$, which they call the limit of "diffused vorticity," although "mean-field" or coarse-grained" vorticity may be more descriptive. A detailed analysis gives the frequencies of the lowest $m= \pm 2$ quadrupole modes in the laboratory frame

$$
\omega(m= \pm 2)=\sqrt{2 \omega_{\perp}^{2}-\Omega^{2}} \pm \Omega .
$$

In particular, the difference of the two frequencies is $2 \Omega$, whereas the sum of the squared frequencies is $4 \omega_{\perp}^{2}$, independent of the rotation rate. Haljan et al. (2001b) fully confirm both these detailed predictions.

As an extension of these experiments, Engels et al. (2002) study the dynamics of the (originally triangular) vortex lattice in a rapidly rotating condensate. They can image in all three perpendicular directions, which allows them to confirm theoretical predictions (Feder and Clark, 2001; García-Ripoll and Pérez-García, 2001b) that the vortices are nearly straight in such dense arrays. Furthermore, Eq. (4.18) shows that the $m= \pm 2$ quadrupole modes have several intriguing features in the limit $\Omega \rightarrow \omega_{\perp}$. In particular, Engels et al. (2002) note that the resulting $m=-2$ mode is small and hence nearly stationary in the laboratory frame, but the corresponding $m=2$ mode is nearly stationary in the rotating frame because $\omega(m=2)^{\prime}=\omega(m=2)-2 \Omega$ then becomes small [see Eq. (3.18)].

In a particularly interesting experiment, Engels et al. (2002) form a rotating vortex lattice with an angular velocity $\Omega / \omega_{\perp} \sim 0.95$. They then apply a relatively small trap distortion with $\left(\omega_{x}^{2}-\omega_{y}^{2}\right) /\left(\omega_{x}^{2}+\omega_{y}^{2}\right) \sim 0.036$ that is stationary in the laboratory. Such a fixed perturbation is nearly resonant with the slow $m=-2$ quadrupole mode. 
As a result, the rotating condensate acquires a stationary quadrupole distortion that grows to $\sim 0.4$ over a time of $\sim 300 \mathrm{~ms}$. Hence the rapidly rotating triangular vortex lattice experiences an oscillating shear distortion with a period that is $\frac{1}{6}$ times the overall rotation period $2 \pi / \Omega$ because of the six-fold symmetry. Every time a basis vector of the rotating lattice lines up with the minor axis of the fixed elliptical confining potential, the vortex lattice deforms significantly, forming what are effectively parallel stripes of closely spaced vortices.

To understand this behavior in detail, recall that the stationary elliptical distortion of the rotating condensate induces a quadrupolar irrotational flow (see the discussion at the beginning of Sec. III.D.2) that combines with the overall rotation $\boldsymbol{v}_{\mathrm{sb}}=\boldsymbol{\Omega} \times \boldsymbol{r}$ to produce the total flow velocity $\boldsymbol{v}$. In the laboratory frame, the vortices follow streamlines of this total local velocity. Cozzini and Stringari (2003) integrate the relevant dynamical equations of motion for the vortices. They indeed find stripe-like patterns whenever one of the lattice vectors lies along the minor axis of the distorted condensate.

In a related approach, Mueller and Ho (2003) use a variational two-dimensional wave function. Although their formalism strictly applies only in the extreme limit of $\Omega \rightarrow \omega_{\perp}$ when the interaction energy is small compared to the single-particle energy (the "lowest Landaulevel" regime, discussed below in Sec. V), their trial wave function can describe a two-dimensional lattice of vortices with general symmetry, using Jacobian elliptic functions that are closely related to those in the earlier work of Tkachenko (1966a). In their simulation, a small elliptical distortion of the trap potential at $t=0$ induces an increasing distortion of the condensate's aspect ratio. The initial triangular vortex array remains a well-defined lattice but deforms following the combined velocity field of the rotational and irrotational flow. As time evolves, stripes appear periodically, producing images that closely resemble those found experimentally (Engels et al., 2002).

\section{Uniformity of the vortex array}

Nearly 30 years ago, Campbell and Ziff (1979) studied numerically the various equilibrium arrangements of two-dimensional vortex arrays in uniform incompressible ideal fluids inside a fixed circular boundary (as a model for superfluid ${ }^{4} \mathrm{He}$ in a rotating bucket). They found predominantly distorted triangular arrangements with concentric circles of vortices and a depleted region near the outer edge. Yarmchuk et al. (1979) see similar patterns in rotating superfluid ${ }^{4} \mathrm{He}$ (see Fig. 8), although the small number of vortices precludes a definitive comparison. In contrast, images of vortex lattices in rotating BECs are strikingly triangular and regular, even out to the edge of the visible condensate, as seen in Fig. 12 .

The treatment of the density profile in the mean-field
Thomas-Fermi regime omits the term $\left(\boldsymbol{v}-\boldsymbol{v}_{\mathrm{sb}}\right)^{2}$ entirely [see Eq. (4.8)], which assumes a uniform vortex distribution and ignores the fine-grain structure of individual vortices. Sheehy and Radzihovsky (2004a,b) investigate these effects by considering a two-dimensional TF condensate with number density $n(\boldsymbol{r})=n(0)\left(1-r^{2} / R_{\perp}^{2}\right)$, where $R_{\perp}$ grows with increasing $\Omega$. This rotating condensate contains an array of vortices at regular twodimensional lattice sites $\boldsymbol{r}_{j}$ with mean density $\bar{n}_{v}=$ $M \Omega /(\pi \hbar)$ [the Feynman value in Eq. (4.1)]. They then subject each vortex to a small displacement field $\boldsymbol{u}(\boldsymbol{r})$, so that the original site moves to $\boldsymbol{r}_{j}+\boldsymbol{u}\left(\boldsymbol{r}_{j}\right)$, and the vortex density becomes

$$
n_{v}(\boldsymbol{r})=\bar{n}_{v}[1-\nabla \cdot \boldsymbol{u}(\boldsymbol{r})]
$$

This distorted vortex density in turn induces a change in the mean background fluid flow based on the following integral

$\overline{\boldsymbol{v}}(\boldsymbol{r}) \approx \frac{\hbar}{M} \int d^{2} r^{\prime} n_{v}\left(\boldsymbol{r}^{\prime}\right) \frac{\hat{\boldsymbol{z}} \times\left(\boldsymbol{r}-\boldsymbol{r}^{\prime}\right)}{\left|\boldsymbol{r}-\boldsymbol{r}^{\prime}\right|^{2}}=\boldsymbol{\Omega} \times \boldsymbol{r}-2 \boldsymbol{\Omega} \times \boldsymbol{u}(\boldsymbol{r})$,

where $\boldsymbol{\Omega}=\Omega \hat{\boldsymbol{z}}$ and the second term is a correction to the solid-body value.

A detailed analysis with Eqs. (4.8), (4.19) and (4.20) expresses the deformation part of the energy $E^{\prime}$ in terms of $\boldsymbol{u}$, and the corresponding Euler-Lagrange equation leads to the general expression

$$
\boldsymbol{u}(\boldsymbol{r}) \approx-\frac{l^{2}}{8} \ln \left(\frac{l^{2}}{\xi^{2}}\right) \nabla \ln n(\boldsymbol{r})
$$

where $n(\boldsymbol{r})$ is the condensate number density, $l$ is the mean circular cell radius from Eq. (4.5), and $\xi$ is evaluated with the central density. ${ }^{8}$ In the particular case of the two-dimensional TF density profile with $n(\boldsymbol{r}) \propto$ $1-r^{2} / R_{\perp}^{2}$, this equation yields

$$
\boldsymbol{u}(\boldsymbol{r}) \approx \frac{l^{2}}{4 R_{\perp}^{2}} \ln \left(\frac{l^{2}}{\xi^{2}}\right) \frac{\boldsymbol{r}}{1-r^{2} / R_{\perp}^{2}} .
$$

Note that the deformation of the regular vortex lattice is purely radial, as might be expected from the circular symmetry, and it increases with increasing distance from the center. Furthermore, the quantity $R_{\perp}^{2} / l^{2}$ is the number $\mathcal{N}_{v}$ of vortices in the rotating TF condensate [see Eq. (4.6)], so that the nonuniform distortion is of order $\mathcal{N}_{v}^{-1}$ (at most a few \%), even though the TF density decreases significantly near the edge of the condensate.

\footnotetext{
8 As a very simple application, assume a uniform particle density, so that $\boldsymbol{u}$ vanishes. Equation (4.19) then indicates that the vortex density is also uniform. Note that this formalism relies on the TF energy functional and has little connection to the numerical studies of vortex arrays in uniform incompressible fluids (Campbell and Ziff, 1979), where a vortex-free region occurs near the outer wall.
} 


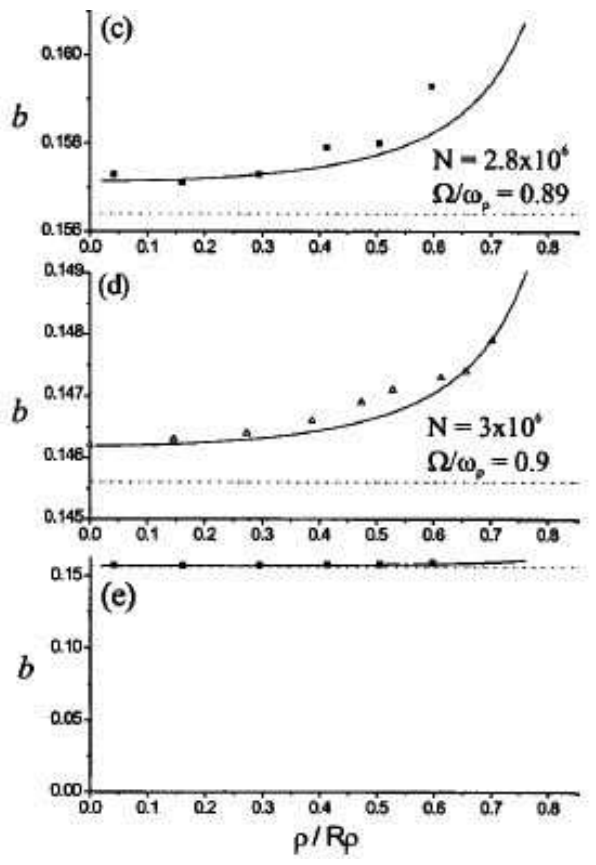

FIG. 13 Lattice spacing as a function of the scaled radial position $r / R_{\perp}$. The solid curve is the prediction (4.24) from Sheehy and Radzihovsky (2004a, b) , with the rigid-body value as a dashed line. Plots (c) and (d) show that the fractional amplitude decreases with increasing $\Omega$, and (e) restores the suppressed zero in (c), indicating that the overall effect is small but measurable. The intervortex spacing increases by less than $2 \%$ over a region where the atomic density changes by $\sim 35 \%$. From Coddington et al. (2004).

Finally, Eq. (4.19) gives the corresponding nonuniform axisymmetric vortex density

$$
n_{v}(r)=\bar{n}_{v}+\frac{1}{8 \pi} \ln \left(\frac{l^{2}}{\xi^{2}}\right) \nabla^{2} \ln n(\boldsymbol{r}),
$$

which becomes

$$
n_{v}(r) \approx \bar{n}_{v}-\frac{1}{2 \pi R_{\perp}^{2}} \ln \left(\frac{l^{2}}{\xi^{2}}\right) \frac{1}{\left(1-r^{2} / R_{\perp}^{2}\right)^{2}},
$$

for the two-dimensional TF condensate. The correction is again small, of order $\mathcal{N}_{v}^{-1}$. Coddington et al. (2004) study this behavior in detail, as shown in Fig. 13, Comparison of images in Fig. 13(c) and 13)(d) show that the distortion decreases with increasing number of vortices (namely with increasing $\Omega$ ), and Fig. 13(e) restores the suppressed zero in (c), indicating that the overall effect is indeed small.

\section{Vortex core size for large rotation speeds}

The principal effect of rapid rotation is the altered aspect ratio seen in Fig. 11, but the rotation also affects the vortex core radius $\xi$. In the TF limit of a large condensate, the basic definition in Eq. (2.31) shows that $\xi^{2}$ increases with increasing $\Omega$ because the radial expansion reduces the central density $n(0)$ and thus the chemical potential $\mu=g n(0)$. To quantify this behavior, it is helpful to consider the dimensionless ratio $\xi^{2} / l^{2}=\hbar \Omega /(2 \mu)$, which is roughly the fraction of each vortex cell that the core occupies. A combination of Eqs. (2.28), (2.29), (2.31), and (4.9) yields the TF expression (Fetter, 2001)

$$
\frac{\xi^{2}}{l^{2}}=\frac{\hbar \Omega}{2 \mu}=\frac{\bar{\Omega}}{\left(1-\bar{\Omega}^{2}\right)^{2 / 5}}\left(\frac{\omega_{\perp}}{\omega_{z}} \frac{d_{\perp}}{15 N a}\right)^{2 / 5},
$$

where $\bar{\Omega}=\Omega / \omega_{\perp}$ is a scaled dimensionless rotation speed.

Since $d_{\perp} /(N a)$ is small for a typical TF condensate, the ratio $\xi^{2} / l^{2}$ also remains small until $\bar{\Omega}$ approaches one, and it then grows rapidly because of the small denominator. As Fischer and Baym (2003) emphasize, this limit of rapid rotation requires a more careful treatment. Specifically, they do not assume that $\xi^{2}$ is proportional to $\mu^{-1}$. Instead, they treat $\xi^{2}$ as a variational parameter and find that the ratio $\xi^{2} / l^{2}$ grows only until it reaches $\approx \frac{1}{2}$, when the vortex core occupies a significant fraction of each unit cell in the vortex lattice. Strictly, the TF limit no longer applies for this large $\bar{\Omega} \lesssim 1$ because the density variation now makes a significant contribution to the total kinetic energy. Baym and Pethick (2004) construct a more general variational theory that includes both the TF limit and the lowest-Landau-level regime described below in Sec. V.

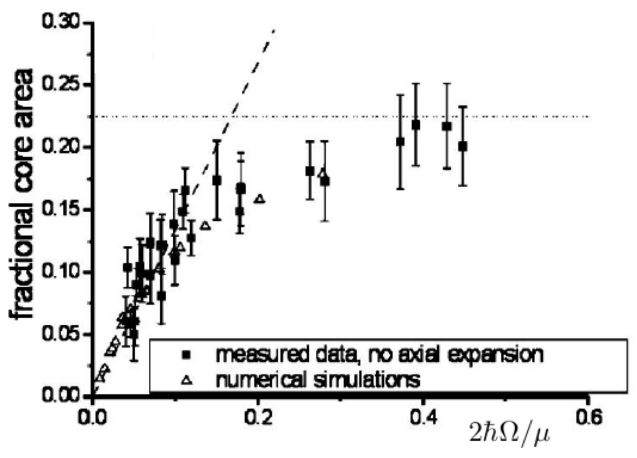

FIG. 14 Fractional vortex core area as function of $2 \hbar \Omega / \mu$. The dashed line is the TF theory and the dotted line is the lowest-Landau-level theory discussed below in Sec. V. From Coddington et al. (2004).

Coddington et al. (2004) study the size of the vortex core from both experimental and numerical perspectives. Because of different fits to the vortex-core density profiles and different definitions of the fractional core area, their numerical values differ somewhat from those in Eq. (4.25) and in Fischer and Baym (2003) and Baym and Pethick (2004). Figure 14 shows typical experimental and numerical values of the fractional core area compared with the ratio $2 \hbar \Omega / \mu$. For small values of $\Omega$, Eq. (4.25) indicates that $\xi^{2} / l^{2}$ should vary linearly with $\Omega / \mu$ in the TF regime. As $\Omega$ increases toward the critical value $\omega_{\perp}$, however, the fractional core area saturates at a constant value. Detailed numerical studies (Cozzini et al., 2006) 
of a rapidly rotating condensate yield a very similar picture of this behavior.

\section{Tkachenko oscillations of the vortex lattice}

In 1966, Tkachenko (1966a b) published two remarkable papers on the behavior of arrays of straight vortices in an unbounded rotating incompressible irrotational fluid (as a model for superfluid ${ }^{4} \mathrm{He}$ ). The first paper shows that a triangular lattice has the lowest energy of all simple lattices (those with one vortex per unit cell). The second paper studies small perturbations of a general lattice, showing that the square lattice is unstable for waves in certain directions, but that the triangular lattice is stable for all normal modes. For the latter structure, he also determines the dispersion relation of the small-amplitude normal modes of a vortex lattice, along with the corresponding eigenvectors for a given wave vector $\boldsymbol{k}$ lying in the $x y$ plane perpendicular to $\boldsymbol{\Omega}$. The calculation is a tour de force of analytic function theory, involving several elliptic-type functions, especially the Weierstrass $\zeta$ function that has a simple pole at each lattice site Chandrasekharan, 1985; Wolfram, 2007). Ultimately, however, the final result is simple. At long wavelengths $k l \ll 1$, where $l=\sqrt{\hbar /(M \Omega)}$ is the vortex-cell radius, the wave is predominantly transverse with a linear dispersion relation

$$
\omega(\boldsymbol{k}) \approx c_{T} k
$$

where

$$
c_{T}=\frac{1}{2} \sqrt{\frac{\hbar \Omega}{M}}=\frac{1}{2} l \Omega
$$

is the speed of propagation. This long-wavelength motion is effectively a transverse phonon in the vortex lattice. ${ }^{9}$

The significant radial expansion of rapidly rotating BECs means that the resulting vortices are essentially two-dimensional. Thus bending modes are irrelevant, but the nonzero compressibility of these atomic gases requires a significant modification to Tkachenko (1966b)'s analysis. Sonin (1987) and Baym (2003) generalize Tkachenko's result in Eq. (4.26) to find the new longwavelength expression

$$
\omega(k)^{2} \approx c_{T}^{2} k^{2} \frac{s^{2} k^{2}}{4 \Omega^{2}+s^{2} k^{2}},
$$

where $s$ is the speed of sound. This expressions assumes an infinite uniform system, but Anglin (2002); Baksmaty et al. (2004); Baym (2003); Sonin (2005) also include the nonuniformity of the trapped BEC. If $s k \gg \Omega$

\footnotetext{
9 Unlike Newtonian particles in a two-dimensional lattice, however, vortices obey first-order dynamical differential equations. Hence, there is only one normal mode for each wave vector $\boldsymbol{k}$.
}

(the short-wavelength or incompressible limit), then this expression reduces to Tkachenko's $\omega(k) \approx c_{T} k$, but if $s k \ll \Omega$ (the long-wavelength or compressible limit), then the mode becomes soft with $\omega(k) \propto k^{2}$. Similar softening of the collective-mode spectrum has important consequences for stability of the vortex lattice at large $\Omega$ (Baym, 2005; Sinova et al., 2002), as discussed in Sec. VI.
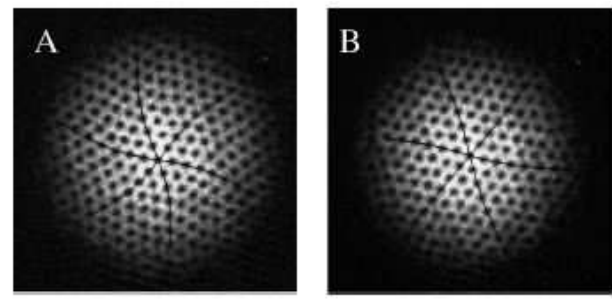

FIG. 15 Lowest Tkachenko mode of the vortex lattice excited by atom removal taken (A) $500 \mathrm{~ms}$ after the end of the blasting pulse and (B) $1650 \mathrm{~ms}$ after the blasting pulse. Lines are sine fits to the distortion of the vortex lattice. From Coddington et al. (2003).

Coddington et al. (2003) observe these Tkachenko oscillations in considerable detail. They start with an essentially uniform vortex lattice and then apply a weak perturbation (along with other approaches, they use a "blasting pulse" that removes a small fraction of the atoms near the center of the trap). At a scaled rotation frequency $\bar{\Omega}=0.95$, they wait a variable time and then turn off the trap, yielding an image of the resulting distorted vortex lattice. Figure 15] shows the vortex lattice at one quarter and three quarters of the oscillation period, with the lines as a sine fit to the distortion of the vortex lattice. In practice, the normal mode has the correct shape, but the measured frequency is significantly smaller than that predicted in Eq. (4.28). Anglin and Crescimanno (2002) suggest that this discrepancy arises from reliance on a continuum theory that ignores the increased vortex core radius for large $\Omega$.

Tkachenko (1969) also evaluates the shear modulus of the triangular vortex lattice in an incompressible fluid, which is directly related to the long-wavelength oscillation spectrum. Subsequently, Cozzini et al. (2006) use numerical methods to study the more general situation of a vortex lattice in a rotating BEC, which also includes both the incompressible (TF) limit and the compressible (LLL) limit (Sinova et al., 2002; Sonin, 2005).

\section{Rotating two-component BECs}

The experimental creation of relatively large vortex arrays in single-components BECs in ${ }^{23} \mathrm{Na}$ (Abo-Shaeer et al., 2001) and ${ }^{87} \mathrm{Rb}$ (Halian et al., 2001b; Hodby et al., 2002; Madison et al., 2000a) rapidly led to proposals for corresponding two-component vortex arrays (Kasamatsu et al., 2003; Mueller and Ho, 2002). 
As discussed in Sec. III.D.1, the interspecies interaction parameter $g_{12}$ significantly affects the behavior, especially for trapped condensates (the intraspecies parameters $g_{1}$ and $g_{2}$ are assumed positive).

Mueller and Ho (2002) initiated the theory of these two-component condensates, assuming rapid rotation $\left(\Omega \lesssim \omega_{\perp}\right)$. This limit allows a considerable simplification (see Sec. V) because the condensate wave functions $\Psi_{1}$ and $\Psi_{2}$ can then be constructed from the lowest-Landaulevel (LLL) single-particle states [the same as those used by Butts and Rokhsar (1999) in Sec. III.C]. Apart from an overall Gaussian factor, the resulting trial solutions $\Psi_{j}$ are analytic functions of the complex variable $\zeta=x+i y$; they involve quasiperiodic Jacobi theta functions that are closely related to other elliptic functions appearing in the treatment of normal modes in a rotating vortex lattice (Chandrasekharan, 1985; Tkachenko, 1966a, b) , as mentioned in Sec. IV.B.4. This analytical approach allows a relatively simple treatment of the change in the vortex lattice as the parameter $\alpha \equiv g_{12} / \sqrt{g_{1} g_{2}}$ varies from negative (when the interspecies interaction is attractive) to positive. Specifically, for negative $\alpha$, the two components prefer to overlap and form a single triangular lattice. As $\alpha$ becomes increasingly positive, however, the overall repulsive interaction energy leads the two components to separate spatially, with interlaced triangular vortex arrays to minimize the overall density variation. Eventually, the triangular lattices should distort, forming square or rectangular arrays when $\alpha \sim 1$.

Subsequently, Kasamatsu et al. (2003) study the behavior at lower angular velocity in the mean-field TF regime. Their extensive numerical analysis of the equilibrium vortex-lattice configuration as a function of the scaled dimensionless rotation rate $\bar{\Omega}=\Omega / \omega_{\perp}$ and the relative interaction strength $\alpha$ gives a qualitatively similar picture: For any reasonable $\bar{\Omega}$, the interpenetrating vortex arrays assume four-fold square symmetry as $\alpha$ approaches $1^{-}$from below.

Schweikhard et al. (2004) perform a series of experiments on these rotating two-component systems $(\bar{\Omega} \approx$ $0.75)$. They start with a single-component triangular vortex array in the state $|1\rangle=\left|F=1, m_{F}=-1\right\rangle$ (compare Sec. III.D.1). A short pulse with the two-photon electromagnetic coupling fields transfers $\sim 80 \%$ of the population to the state $|2\rangle=\left|F=2, m_{F}=1\right\rangle$. The initial triangular vortex lattice is now unstable, and after a turbulent stage $(\sim 2 \mathrm{~s})$, it transforms to a square structure that persists for another $\sim 4 \mathrm{~s}$. Ultimately, however, the state $|2\rangle$ decays through a spin-flip transition. As a result, the remaining single-component condensate in state |1) again transforms back to a triangular array, similar to the initial state.

\section{Rotating gas of paired fermions in tightly bound (BEC) limit}

One of the many remarkable new results from the study of ultracold gases is the superfluidity of bound pairs of fermionic atoms such as ${ }^{6} \mathrm{Li}$ and ${ }^{40} \mathrm{~K}$. Although a detailed discussion is inappropriate here, Secs. I and VIII of Bloch et al. (2007), Sec. III of Giorgini et al. (2007), and Sec. 8.4 of Leggett (2006) provide excellent treatments of the behavior near a Feshbach resonance. Here, I simply review the basic elements of scattering theory to explain how the $s$-wave scattering length can exhibit a resonance associated with the appearance of a new bound state.

The simplest description of a dilute atomic BEC assumes a weak repulsive effective interaction $g>0$ with a positive $s$-wave scattering length $a>0$ (this picture holds for the bosonic atoms ${ }^{87} \mathrm{Rb}$ and ${ }^{23} \mathrm{Na}$ ). Such a system is stable both in bulk and in a trap, with a variety of associated collective modes. In contrast, the bosonic atom ${ }^{7} \mathrm{Li}$ has an attractive effective interaction $g<0$ with a negative $a<0$. In bulk, the negative interaction would yield an imaginary speed of sound, implying a long-wavelength instability. In a trap, however, the positive kinetic and potential energy can stabilize the condensate for sufficiently small $N$, but the system will collapse (Bradley et al., 1997; Ruprecht et al., 1995) above a critical $N_{c}$. As an alternative picture of this stabilization, note that the quantized wavenumbers in a trap provide a lower bound $k_{\min } \sim \pi / R$, where $R$ depends on $N$. If the discrete analog of the Bogoliubov energy spectrum is real for all $k \geq k_{\text {min }}$, then the trapped condensate is stable even for negative $a$.

The direct relation between the sign of $g$ and the sign of $a$ holds only for weak potentials that have no bound states. The situation becomes more interesting when the potential can support one (or more) $s$-wave bound states. In this case, the $s$-wave scattering length exhibits divergent resonant behavior. To be very specific, let us consider a spherical square-well potential $V(r)=V_{0} \theta(b-r)$ with height $V_{0}$ and range $b$, where $\theta$ denotes the unit positive step function.

1. Bound states occur only for negative $V_{0}=-\left|V_{0}\right|$. A standard analysis [see, for example, Sec. 33 of Landau and Lifshitz (1965) and pp. 164 and 349 of Shankar (1994)] shows that there are no $s$-wave bound states if $\left|V_{0}\right|<\pi^{2} \hbar^{2} /\left(8 M b^{2}\right)$; as $\left|V_{0}\right|$ increases, the $n$th $s$-wave bound state appears when

$$
\left|V_{0}\right|=\frac{(2 n-1)^{2} \pi^{2} \hbar^{2}}{8 M b^{2}}
$$

2. Consider now the scattering of a particle with positive energy $E=\hbar^{2} k^{2} /(2 M)$. The radial $l=$ 0 wave function $\psi(r)=u(r) / r$ has the asymptotic form $u(r)=\sin (k r+\delta)$, where $\delta(k)$ is the $s$-wave phase shift. A detailed analysis for the same square-well potential $V(r)$ [see, for example Sec. 130 of Landau and Lifshitz (1965) and pp. 549554 of Shankar (1994)] yields the explicit expression

$$
\delta=-k b+\arctan \left(\frac{k}{k^{\prime}} \tan k^{\prime} b\right)
$$


where $k^{\prime 2}=2 M\left(E-V_{0}\right) / \hbar^{2}$ determines the wavenumber $k^{\prime}$ inside the square well. ${ }^{10}$ It is evident physically that $\delta$ is negative for positive $V_{0}$ because the repulsive potential pushes out the wave function, whereas $\delta$ is positive for negative $V_{0}$ because the attractive potential pulls in the wave function. This phase shift completely characterizes the $s$-wave scattering by this potential.

It is conventional and convenient to introduce the $s$-wave scattering length $a \equiv-\lim _{k \rightarrow 0} \delta(k) / k$; for the square-well potential, this result gives

$$
a=b-\frac{\tan k^{\prime} b}{k^{\prime}},
$$

where $k^{2}=-2 M V_{0} / \hbar^{2}$ is now evaluated for $k=0$ (and hence $E=0) .{ }^{11}$ If $V_{0}$ is positive and large, then $a \rightarrow b$, but the dependence can be very different in other situations. For example, $a$ vanishes if $V_{0}=0$ (as expected). More significant is the behavior for negative $V_{0}$, especially near the appearance of a bound state. Equation (4.29) shows that $k^{\prime} b$ increases through $\left(n-\frac{1}{2}\right) \pi$ when the $n$th $s$-wave bound state first forms. Consequently, Eq. (4.31) diverges to negative infinity when the state is just unbound and then decreases from positive infinity when the trapped state is weakly bound. Such resonant dependence is a typical and generic feature of scattering by an attractive potential that can support one or more bound states.

In the context of dilute ultracold gases, the attractive long-range van der Waals potential acts to bind the two fermionic atoms to form a molecule. In general, there are several molecular states, each with different magnetic moments. Thus an applied magnetic field can shift a bound state in a closed channel across the asymptotic continuum in an open channel, leading to a "Feshbach resonance" [see, for example, Moerdiik et al. (1995), Köhler et al. (2006), Sec. 2 of Castin (2006) and Secs. I and VIII of Bloch et al. (2007) for more detailed discussions]. In essence, sweeping an external magnetic field through a Feshbach resonance transforms a tightly bound bosonic molecule of the two fermions into a weakly attracting fermionic pair. The bosonic molecules can form a BEC that displays essentially all the properties of an atomic BEC, whereas the weakly attracting fermionic pairs are analogous to the Cooper pairs in the BCS theory of conventional low-temperature superconductors (de Gennes, 1966; Tinkham, 1996). As a result, this transition through a Feshbach resonance is called the "BEC-BCS crossover."

10 If $V_{0}>E$, then $k^{\prime}$ is written as $i \kappa$ and $\tan k^{\prime} b / k^{\prime}$ becomes $\tanh \kappa b / \kappa$.

11 If $V_{0}$ is positive, then this relation is replaced by $\kappa^{2}=2 M V_{0} / \hbar^{2}$; correspondingly, Eq. 4.31) becomes $a=b-\tanh \kappa b / \kappa$.

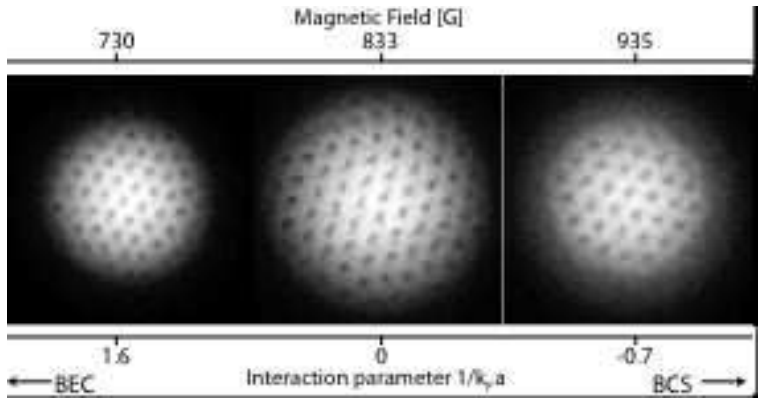

FIG. 16 Vortex lattice in a rotating gas of ${ }^{6} \mathrm{Li}$ fermions. The left figure is on the BEC side, the central figure is on resonance, and the right figure is on the BCS side [note that the horizontal scale is $-\left(k_{F} a\right)^{-1}$, which is effectively $-\left(n^{1 / 3} a\right)^{-1}$ apart from a numerical factor]. From Zwierlein (2007).

Particularly relevant in the present context is the experiment of Zwierlein et al. (2005) who rotate a BEC of tightly bound bosonic molecules with a deformed optical trap (the magnetically induced Feshbach resonance precludes the use of the usual magnetic trap). They then sweep the magnetic field from the positive scattering length (the BEC side of bound molecules) through the resonance to the negative scattering length (the BCS side of attracting fermionic pairs). Figure 16 shows the vortex lattice at three different values of the magnetic field. Note that the scattering length $a$ diverges in the resonance region. Consequently, it is conventional to use $\left(k_{F} a\right)^{-1}$ as the independent variable, which varies smoothly from positive in the BEC limit through zero near the crossover region to negative in the BCS limit. Here, $k_{F}$ is the Fermi wavenumber, but, apart from a numerical factor of order unity, it is the same as $n^{1 / 3}$, with $n$ the boson density. The explicit persistence of quantized vortices through the BEC-BCS crossover provides strong evidence for superfluidity on the fermionic side, although the experiment did not reach the weakly bound BCS limit [Sec. VIII of Giorgini et al. (2007) gives a detailed discussion of rotating ultracold Fermi gases].

Sensarma et al. (2006) use the fermionic Bogoliubovde Gennes equations [see, for example, Chap. 5 of de Gennes (1966) and Sec. 10.1 of Tinkham (1996)] to study the structure of a single quantized vortex in a uniform three-dimensional atomic Fermi gas at zero temperature. Here I consider only the BEC side, since a full summary of ultracold Fermi gases is beyond the scope of this review.

Let us focus on the vortex core radius (healing length) $\xi$ defined in Eq. (2.31) for a trapped condensate. As noted in Sec. IV.B.3, $\xi$ grows with increasing rotation speed $\Omega$ because of the reduced central density [and hence reduced chemical potential $\mu(\Omega)$ ], but Eq. (4.25) also exhibits the explicit dependence on the scattering length $a$. Here, it is preferable to consider the slightly different 
dimensionless ratio

$$
\frac{\xi}{d_{0}}=\frac{1}{\left(1-\bar{\Omega}^{2}\right)^{1 / 5}}\left(\frac{d_{0}}{15 N a}\right)^{1 / 5},
$$

which involves only the scaled rotation speed $\bar{\Omega}=\Omega / \omega_{\perp}$, the $s$-wave scattering length $a$, and the trap geometry through $d_{0}$ and the radial rap frequency $\omega_{\perp}$.

Sensarma et al. (2006) remark that the healing length in a uniform cold BEC Eq. (2.21) is proportional to $(n a)^{-1 / 2}$ and decreases with increasing positive $a$ for fixed $n$. Consequently, the vortex core size should shrink on approaching the resonance regime from the BEC side. Note, however, that inclusion of the trap in Eq. (4.32) alters the fractional power from $a^{-1 / 2}$ to $a^{-1 / 5}$. Thus the dependence on a may be difficult to measure in current experiments. It is also clear from Eq. (2.22) that the GP picture fails as $\xi$ approaches the interparticle spacing, which is expected to be the minimum value for $\xi$. On the BCS side, the qualitative relation $\xi \sim \hbar v_{F} / \Delta$ shows that the vortex core again grows on moving away from the resonance regime because the BCS energy gap $\Delta$ then becomes small (Sensarma et al., 2006) [see also Sec. 8.4 of Leggett (2006)].

On the BEC side, recall that the condensate density $n(r)$ around a vortex decreases rapidly from its bulk value $n$ for $r \lesssim \xi$ inside the vortex core. Consequently, the circulating mass current $j(r)=M n(r) v(r)=n(r) \hbar / r$ reaches a maximum "critical" value $j_{c} \sim n \hbar / \xi$ for $r \approx \xi$ [compare the discussion below Eq. (3.4)]. As $a$ increases on the BEC side toward the Feshbach resonance, the decreased $\xi$ implies that this critical mass current $j_{c}$ grows and saturates when $\xi \sim n^{-1 / 3}$. A more detailed analysis by Sensarma et al. (2006) shows that $j_{c}$ then again decreases on moving from the resonance regime to the BCS side. Thus both $\xi$ and $j_{c}$ display non-monotonic dependence in traversing the BEC-BCS crossover region.

\section{Effect of additional quartic confinement}

The discussion of rotating condensates in the meanfield Thomas-Fermi regime (Sec. IV.A) makes it clear the a purely harmonic trap with radial frequency $\omega_{\perp}$ will cease to confine the system if the rotation speed $\Omega$ reaches or exceeds $\omega_{\perp}$. Indeed, the limit $\Omega \rightarrow \omega_{\perp}$ is singular, because the condensate radius and the total angular momentum both diverge. In the regime $\omega_{\perp}-\Omega \ll \omega_{\perp}$, the system is expected to undergo a series of crossovers and complicated phase transitions (Secs. V and VI) whose exact nature remains under debate.

Thus it is interesting to consider the effect of adding an additional strongly confining potential that rises more rapidly than $r^{2}$. I here focus on the most common (quartic) model $\propto r^{4}$ (Fetter, 2001; Kasamatsu et al. , 2002; Kavoulakis and Baym, 2003; Lundh, 2002). Such an anharmonic trap will confine the condensate even for $\Omega>\omega_{\perp}$, thus allowing a more controlled study of possible new states (they are not generally the same as those predicted in a harmonic trap in the limit $\Omega \rightarrow \omega_{\perp}$ ). In terms of the usual dimensional quantities, the anharmonic radial trap potential has the form

$$
V_{\mathrm{tr}}(r)=\frac{\hbar \omega_{\perp}}{2}\left(\frac{r^{2}}{d_{\perp}^{2}}+\lambda \frac{r^{4}}{d_{\perp}^{4}}\right),
$$

where $d_{\perp}=\sqrt{\hbar /\left(M \omega_{\perp}\right)}$ is the radial oscillator length and $\lambda$ is a dimensionless parameter that characterizes the admixture of quartic contribution.

For simplicity, I focus on the limit of rapid rotation and consider only a two-dimensional condensate that is uniform in the $z$ direction over a length $Z$ (Fetter et al., 2005; Kavoulakis and Baym, 2003), with $\Psi(\boldsymbol{r}, z)=\psi(\boldsymbol{r}) / \sqrt{Z}$. It is also convenient to measure energies in units of $\hbar \omega_{\perp}$ and lengths in units of $d_{\perp}$, and to normalize the dimensionless condensate wave function $\psi$ with $\int d^{2} r|\psi|^{2}=N$. In the mean-field TF regime, the dimensionless energy functional in the rotating frame [compare Eq. [4.7)] becomes

$$
\begin{array}{r}
E^{\prime}[\psi]=\int d^{2} r\left[\frac{1}{2}\left(v^{2}+r^{2}+\lambda r^{4}\right)|\psi|^{2}\right. \\
\left.-\boldsymbol{\Omega} \cdot \boldsymbol{r} \times \boldsymbol{v}|\psi|^{2}+\frac{1}{2} \bar{g}|\psi|^{4}\right],
\end{array}
$$

where $\bar{g}=4 \pi a / Z$ is a dimensionless two-dimensional interaction parameter. An alternative model for the axial confinement uses a tight harmonic trap, in which case the interaction parameter is $\bar{g}=\sqrt{8 \pi} a / d_{z}$, but the subsequent description is unaffected.

When the rotating condensate has many vortices, the total velocity $\boldsymbol{v}$ closely approximates the solid-body form $\boldsymbol{\Omega} \times \boldsymbol{r}$, in which case Eq. (4.34) reduces to

$$
E^{\prime}[\psi]=\int d^{2} r\left\{\frac{1}{2}\left[\left(1-\Omega^{2}\right) r^{2}+\lambda r^{4}\right]|\psi|^{2}+\frac{1}{2} \bar{g}|\psi|^{4}\right\} .
$$

As in Eq. (2.25), variation with respect to $|\psi|^{2}$ at fixed normalization yields two-dimensional density

$$
\bar{g}|\psi(r)|^{2}=\mu+\frac{1}{2}\left[\left(\Omega^{2}-1\right) r^{2}-\lambda r^{4}\right] .
$$

For $\Omega<1$, the density has a local maximum at the center, but it changes to a local minimum for $\Omega>1$. In addition, the central density $|\psi(0)|^{2}$ is proportional to the chemical potential $\mu$. As in the previous Eq. (4.9), $\mu$ depends on $\Omega$ through the normalization and decreases continuously with increasing $\Omega$, reflecting the radial expansion of the condensate.

In contrast to the mean-field TF picture for a harmonic radial trap, however, the chemical potential here can vanish at a critical angular velocity $\Omega_{h}>1$, when the central density also vanishes. This behavior indicates the formation of a central hole; for $\Omega>\Omega_{h}$, the chemical potential $\mu$ becomes negative and the condensate assumes an annular form [see (Fetter et al., 2005) for a detailed description of this unusual structure].

As $\Omega$ continues to increase, the system can, in principle, make a second transition to a different annular 
state with purely irrotational (vortex-free) circulating flow, known as a "giant vortex." In this case, the circulating velocity $v(r)$ has the quantized form $\mathcal{N}_{v} / r$, reflecting the presence of $\mathcal{N}_{v}$ phantom vortices in the hole (equivalently, the flow simply represents $\mathcal{N}_{v}$-fold quantized circulation in a multiply connected condensate). Kavoulakis and Baym (2003) suggest the possibility of such irrotational flow in a quadratic plus quartic potential [earlier, Fischer and Baym (2003) propose a similar structure in a rotating rigid cylinder]. As noted at the start of Sec. IV, solid-body rotation [the model used in obtaining Eq. (4.36)] gives the absolute minimum energy for a rotating system. Consequently, any irrotational giant vortex necessarily depends on the finite quantization of circulation that introduces graininess in the superflow. In practice, estimates of the critical angular velocity for formation of a giant vortex require considerable numerical work [see (Fetter et al., 2005; Fu and Zaremba, 2006; Kavoulakis and Bavm, 2003; Kim and Fetter, 2005) for more details].

In an elegant series of experiments, the ENS (Paris) group (Bretin et al., 2004; Stock et al., 2004) study the effect of a weak quartic confinement in addition to the usual quadratic trap potential [see also (Stock et al., 2005a) for a more general review of rotating BECs]. Specifically, they use a blue-detuned (repulsive) Gaussian laser beam propagating along the symmetry axis of their elongated condensate, with a resulting dipole potential

$$
V_{\mathrm{d}}(r)=V_{0} e^{-2 r^{2} / w^{2}} \approx V_{0}-\frac{2 V_{0}}{w^{2}} r^{2}+\frac{2 V_{0}}{w^{4}} r^{4}+\cdots .
$$

The positive constant term $V_{0}$ simply shifts to zero of energy, the quadratic term reduces the original radial trap frequency, and the quartic term provides weak positive confinement. The measured oscillation frequency of the center of mass of the condensate gives the effective radial trap frequency $\omega_{\text {eff }} / 2 \pi=64.8 \mathrm{~Hz}$ (Stock et al., 2004), and the other trap parameters yield the quartic coupling constant $\lambda \approx 10^{-3}$, indicating that the added confinement in Eq. (4.33) is indeed weak.

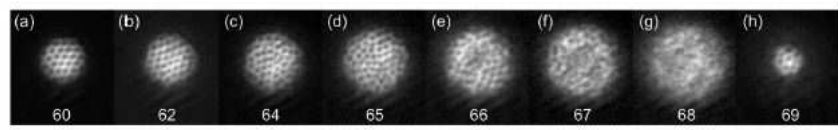

FIG. 17 Pictures of the rotating gas taken along the rotation axis. The number below each picture is the rotation frequency in $\mathrm{Hz}$ (note that $\omega_{\text {eff }} / 2 \pi=64.8 \mathrm{~Hz}$ is the effective radial trap frequency). Taken from (Bretin et al., 2004).

For the dimensionless rotation speed $\Omega \approx 1$, the rotational deformation produces a nearly spherical condensate that remains stable up to $\Omega \lesssim 1.05$ (Bretin et al. , 2004). Figure 17 shows the expanded cloud for various rotation speeds both below and above the critical value $\omega_{\text {eff }}$ Initially, a regular vortex array appears, but it becomes irregular in Figs. 17(d)-(f). In addition, for
$\Omega>\omega_{\text {eff }}$, the condensate exhibits a clear local minimum in the density near the center, as expected from Eq. (4.36) in the mean-field TF picture. Throughout the analysis, they fit the measured condensate shape to the TF prediction, which provides a direct determination of $\Omega$. They also use surface-wave spectroscopy as discussed in Sec. III.D.3 (Cozzini and Stringari, 2003; Svidzinsky and Fetter, 1998; Zambelli and Stringari, 1998) to obtain an independent measure of $\Omega$ (even though the number of visible vortices appears to be too small for $\Omega \geq \omega_{\text {eff }}$.

The absence of visible vortices in Fig. 17( $(\mathrm{g})$ is puzzling. One possible reason for the discrepancy is the three-dimensional character of the condensate, which allows the vortices to bend. Also, the low temperature may make it difficult for the system to equilibrate. Numerical studies (Aftalion and Danaila, 2004) suggest that much longer times are needed to attain a well-ordered vortex lattice in these regimes of large $\Omega$. In any case, Fig. 17(h) implies that the condensate simply collapses, although the reason remains unknown.

\section{VORTEX ARRAYS IN MEAN-FIELD LOWEST-LANDAU-LEVEL (LLL) REGIME}

In the mean-field Thomas-Fermi description of a rotating BEC, the basic approximation is the neglect of the "kinetic energy" associated with the density variations. Specifically, Eq. (4.7) omits the term $\hbar^{2}(\nabla|\Psi|)^{2} /(2 M)$ whereas the usual kinetic energy from the phase variation $\frac{1}{2} M v^{2}|\Psi|^{2}$ remains. As seen in Eq. (4.25), the vortex cores are small for moderate values of the dimensionless rotation speed $\bar{\Omega}=\Omega / \omega_{\perp}$, so that this approximation is valid. As $\bar{\Omega}$ increases toward 1 , however, it fails when $1-\bar{\Omega} \sim d_{\perp} /(15 N a) \ll 1$ because the vortex cores then become comparable with the intervortex separation $\sim 2 l$. In this case, it becomes essential to return to the full GP energy functional $E^{\prime}[\Psi]$ in Eq. (3.6).

In thinking about this new regime, $\mathrm{Ho}$ (2001) observes that the interaction energy per particle [of order $\mu(\Omega)=g n(0)]$ is small because the condensate expands radially [see Eq. (4.9)]. In essence, the condensate becomes two-dimensional, and it is convenient to study a two-dimensional condensate that is uniform in the $z$ direction over a length $Z$. Thus the original condensate wave function $\Psi(\boldsymbol{r}, z)$ can be written as $\psi(\boldsymbol{r}) / \sqrt{Z}$, where $\psi(\boldsymbol{r})$ is a two-dimensional wave function with normalization $\int d^{2} r|\psi|^{2}=N$. The GP energy functional then becomes

$$
\begin{array}{r}
E^{\prime}[\psi]=\int d^{2} r \psi^{*}\left(\frac{p^{2}}{2 M}+\frac{1}{2} M \omega_{\perp}^{2} r^{2}-\Omega L_{z}\right. \\
\left.+\frac{1}{2} g_{2 D}|\psi|^{2}\right) \psi
\end{array}
$$

where $g_{2 D}=g / Z$. As in Sec. IV.C, one can instead assume a tight Gaussian axial confinement, in which case $g_{2 D}=g /\left(\sqrt{2 \pi} d_{z}\right)$, with $d_{z}=\sqrt{\hbar /\left(M \omega_{z}\right)}$. 


\section{A. Physics of lowest-Landau-level (LLL) one-body states}

The one-body Hamiltonian in Eq. (5.1) has the crucial advantage that it is exactly soluble with very simple energy eigenvalues [see, for example, Sec. 12.14 of Messiah (1961) and pp. 727-741 of Cohen-Tannoudii et al. (1977)]. Consider the (nonrotating) two-dimensional isotropic harmonic oscillator with Hamiltonian

$$
H_{0}=\frac{p^{2}}{2 M}+\frac{1}{2} M \omega_{\perp}^{2} r^{2} .
$$

Classically, the motion is that of two independent oscillators (along $x$ and $y$ ) that can combine to give, for example, circular motion with frequency $\omega_{\perp}$ in the positive (counterclockwise) or negative (clockwise) sense, depending on the relative phases. When viewed from a frame that rotates with angular velocity $\Omega$ in the positive sense, the two circular orbits with originally degenerate frequencies now have different frequencies

$$
\omega_{ \pm}=\omega_{\perp} \mp \Omega
$$

since the positive (negative) mode has a reduced (increased) frequency in the rotating frame.

The quantum-mechanical description relies on the familiar creation and annihilation operators

$a_{x}=\frac{1}{\sqrt{2}}\left(\frac{x}{d_{\perp}}+i \frac{p_{x} d_{\perp}}{\hbar}\right), \quad a_{x}^{\dagger}=\frac{1}{\sqrt{2}}\left(\frac{x}{d_{\perp}}-i \frac{p_{x} d_{\perp}}{\hbar}\right)$,

along with similar operators $a_{y}$ and $a_{y}^{\dagger}$. In the present case, it is more appropriate to use circularly polarized states with operators

$$
a_{ \pm}=\frac{a_{x} \mp i a_{y}}{\sqrt{2}}, \quad a_{ \pm}^{\dagger}=\frac{a_{x}^{\dagger} \pm i a_{y}^{\dagger}}{\sqrt{2}}
$$

that obey the usual commutation relations $\left[a_{+}, a_{+}^{\dagger}\right]=$ $\left[a_{-}, a_{-}^{\dagger}\right]=1$, with all other commutators vanishing. A straightforward analysis [Sec. 12.14 of Messiah (1961) and pp. 727-741 of Cohen-Tannoudji et al. (1977)] expresses $H_{0}$ in terms of these new operators

$$
H_{0}=\hbar \omega_{\perp}\left(a_{+}^{\dagger} a_{+}+a_{-}^{\dagger} a_{-}+1\right) .
$$

Similarly the angular momentum $L_{z}=x p_{y}-y p_{x}$ becomes

$$
L_{z}=\hbar\left(a_{+}^{\dagger} a_{+}-a_{-}^{\dagger} a_{-}\right)
$$

The number operators $a_{ \pm}^{\dagger} a_{ \pm}$have non-negative integer eigenvalues $n_{ \pm}$; the corresponding operators $a_{+}^{\dagger}$ and $a_{+}$ ( $a_{-}^{\dagger}$ and $a_{-}$) create and destroy one quantum with positive (negative) circular polarization and one unit of positive (negative) angular momentum. For the nonrotating system with Hamiltonian $H_{0}$, each quantum simply has an energy $\hbar \omega_{\perp}$.
The principal advantage of this particular circularly polarized basis appears for the rotating system, with Hamiltonian $H_{0}^{\prime}=H_{0}-\Omega L_{z}$, because Eqs. (5.6) and (5.7) together imply

$$
H_{0}^{\prime}=\hbar \omega_{\perp}+\hbar \omega_{+} a_{+}^{\dagger} a_{+}+\hbar \omega_{-} a_{-}^{\dagger} a_{-},
$$

where $\omega_{ \pm}=\omega_{\perp} \mp \Omega$ are the frequencies in Eq. (5.3) for the positive and negative classical orbits as seen in the rotating frame. Correspondingly, the energy eigenvalues are labeled with two non-negative integers $n_{ \pm}$

$$
\epsilon\left(n_{+}, n_{-}\right)=n_{+} \hbar\left(\omega_{\perp}-\Omega\right)+n_{-} \hbar\left(\omega_{\perp}+\Omega\right),
$$

where I omit the zero-point energy $\hbar \omega_{\perp}$ for simplicity. In the limit of rapid rotations $\left(\Omega \rightarrow \omega_{\perp}\right)$, these eigenvalues are essentially independent of $n_{+}$, which implies a large degeneracy. The other integer $n_{-}$then becomes the Landau-level index, with different Landau levels separated by an energy gap of $\sim 2 \hbar \omega_{\perp}$.

For small angular velocity, it is often convenient to introduce a different set of quantum numbers [pp. 727741 of Cohen-Tannoudji et al. (1977)] $n=n_{+}+n_{-}$for the energy and $m=n_{+}-n_{-}$for the angular momentum, so that the energy eigenvalues have the equivalent form

$$
\epsilon_{n m} \equiv \epsilon\left[\frac{1}{2}(n+m), \frac{1}{2}(n-m)\right]=n \hbar \omega_{\perp}-m \hbar \Omega .
$$

Figure [18 uses this new basis to illustrate the energy spectrum $\epsilon_{n m}$ for different values of $\Omega$.

1. On the left side of the figure $(\Omega=0)$, the excitation energy is simply $n \hbar \omega_{\perp}$, independent of $m$, forming an inverted pyramid of states: for each non-negative integer $n$, there are $n+1$ degenerate angular-momentum states ranging from $-n$ to $n$ in steps of 2 . For small $\bar{\Omega} \ll 1$, the energy quantum number $n$ determines the large splitting and the angular-momentum quantum number $m$ lifts the remaining degeneracy (physically, this Coriolisinduced splitting is like the weak-field Zeeman splitting of magnetic sublevels).

2. The central figure shows the situation for moderate $\Omega$, where the (now signficant) Coriolis effect eliminates the degeneracy of the nonrotating spectrum. States with $m=0$ are unshifted, states with negative $m$ shift up by $|m| \hbar \Omega$, whereas those with positive $m$ shift down by the same amount.

3. The right side shows the extreme situation for $\Omega \rightarrow$ $\omega_{\perp}$, when the states again become nearly degenerate, forming essentially horizontal rows (the Landau levels). The lowest Landau level has $n=m$, which means $n_{-}=0$ and $n_{+}=m$; the first excited Landau level has $n-m=1$ (namely $n_{-}=1$ and $\left.n_{+}=m+1\right)$, etc. In this limit $(\bar{\Omega} \lesssim 1)$, the quantum numbers $n_{+}$and $n_{-}$of the rotating basis are most convenient. 

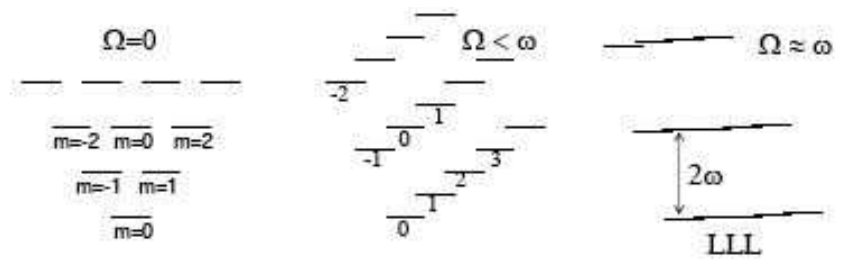

FIG. 18 Excitation energy levels $\epsilon_{n m}=n \hbar \omega_{\perp}-m \Omega$ from Eq. (5.10) for various angular velocity $\Omega$ expressed in terms of quantum numbers $n=n_{+}+n_{-}$for energy and $m=n_{+}-n_{-}$ for angular momentum. Left side is for $\Omega=0$, showing an inverted pyramid of states: for each non-negative integer $n$, there are $n+1$ degenerate angular-momentum states ranging from $-n$ to $n$ in steps of 2 . Center shows the situation for moderate $\Omega$. States with $m=0$ are unshifted, states with negative $m$ shift up whereas those with positive $m$ shift down. Right side shows the extreme situation for $\Omega \rightarrow \omega_{\perp}$, when the states form essentially horizontal rows (the Landau levels). The lowest Landau level has $n=m$, which means $n_{-}=0$, the first excited Landau level has $n-m=1$ (namely $n_{-}=1$ ), etc. From Dalibard (2007).

For rapid rotation $(\bar{\Omega} \lesssim 1)$, it is appropriate to focus on states in the lowest Landau level (those with $n_{-}=0$, denoted LLL), when the single quantum number $n_{+}=m$ suffices to specify the one-body states. The relevant energies are the gap $2 \hbar \omega_{\perp}$ between adjacent Landau levels and the mean interaction energy $g n(0)=\mu$, which is assumed small in the present limit. Thus the basic expansion parameter of the theory is $\mu /\left(2 \hbar \omega_{\perp}\right)$, although current experiments have only achieved values as small as 0.6 (Schweikhard et al., 2004). The corresponding twodimensional eigenfunctions $\psi_{m}(\boldsymbol{r})$ of both $H_{0}^{\prime}$ and $L_{z}$ have a very simple form in plane-polar coordinates [note that these are precisely the trial states from Eq. (3.19) used to study vortex arrays in a weakly interacting condensate (Butts and Rokhsar, 1999)]

$$
\psi_{m}(\boldsymbol{r}) \propto r^{m} e^{i m \phi} e^{-r^{2} /\left(2 d_{\perp}^{2}\right)},
$$

where the normalization is unimportant for the present purpose.

In particular, the ground state $\psi_{0}$ is just a twodimensional isotropic Gaussian. It represents the vacuum for both circularly polarized modes

$$
a_{ \pm} \psi_{0}=0 .
$$

The higher states $\psi_{m}$ for positive $m$ are proportional to $\left(a_{+}^{\dagger}\right)^{m} \psi_{0}$; they can be written in terms of $\zeta=(x+$ $i y) / d_{\perp}=\left(r / d_{\perp}\right) e^{i \phi}$ as $\psi_{m} \propto \zeta^{m} e^{-r^{2} /\left(2 d_{\perp}^{2}\right)}$, where the first factor is just a non-negative integral power of the dimensionless complex variable $\zeta$. For a given $m \gg 1$, the density $\left|\psi_{m}(\boldsymbol{r})\right|^{2}$ is large only in a circular strip of radius $\sim \sqrt{m} d_{\perp}$ and width $\sim d_{\perp} / \sqrt{m}$. In addition, it is easy to see that the mean-square radius for any nonnegative $m$ is given by

$$
\left\langle r^{2}\right\rangle_{m}=(m+1) d_{\perp}^{2} .
$$

Since the same LLL wave functions appear in the fractional quantum Hall effect for two-dimensional electrons in a strong magnetic field, this mean-field LLL regime is sometimes called the "mean-field quantum Hall" regime (Watanabe et al., 2004). In fact, related many-body states from the fractional quantum Hall effect play an important role for a rotating dilute Bose gas at still higher angular velocity, where the physics will turn out to be quite different (Sec. VI). Thus it seems preferable to reserve the name "quantum Hall regime" for this more unusual (nonsuperfluid) limit.

\section{B. Direct treatment of LLL states}

Before using the LLL wave function to construct a GP condensate wave function, I briefly discuss an alternative approach to the LLL states. It is natural to rewrite the single-particle Hamiltonian in the rotating frame $H_{0}^{\prime}=$ $p^{2} /(2 M)+\frac{1}{2} M \omega_{\perp}^{2} r^{2}-\boldsymbol{\Omega} \cdot \boldsymbol{r} \times \boldsymbol{p}$ by completing the square to obtain two equivalent forms

$$
\begin{aligned}
& H_{0}^{\prime}=\frac{(\boldsymbol{p}-M \boldsymbol{\Omega} \times \boldsymbol{r})^{2}}{2 M}+\frac{1}{2} M\left(\omega_{\perp}^{2}-\Omega^{2}\right) r^{2}(5 \\
& H_{0}^{\prime}=\frac{\left(\boldsymbol{p}-M \boldsymbol{\omega}_{\perp} \times \boldsymbol{r}\right)^{2}}{2 M}+\left(\boldsymbol{\omega}_{\perp}-\boldsymbol{\Omega}\right) \cdot \boldsymbol{L},
\end{aligned}
$$

where $\boldsymbol{\omega}_{\perp}=\omega_{\perp} \hat{\boldsymbol{z}}$. Either form is reminiscent of the nonrelativistic Hamiltonian of a particle with charge $q$ in a magnetic field $\boldsymbol{B}$, where the momentum $\boldsymbol{p}$ and vector potential $\boldsymbol{A}$ appear in the gauge-invariant combination $\boldsymbol{p}-q \boldsymbol{A}$, with $\boldsymbol{B}=\boldsymbol{\nabla} \times \boldsymbol{A}$.

Landau solved the quantum problem of an electron in a uniform magnetic field $\boldsymbol{B}$ [see, for example, Chap. XV of Landau and Lifshitz (1965), but the vector potential has a different (asymmetric) form $\boldsymbol{A}=-B y \hat{\boldsymbol{x}}]$. In contrast, pp. 742-764 of Cohen-Tannoudii et al. (1977) solve the same problem in the symmetric gauge $\boldsymbol{A}=\frac{1}{2} \boldsymbol{B} \times \boldsymbol{r}$. It is of course possible simply to quote those results to obtain the LLL wave functions $\psi_{m}$, but such an approach requires a charge $q$, whose sign (positive or negative) determines some crucial conventions in the choice of quantum-mechanical states. As written, Eq. (5.15) suggests a unit positive charge with fictitious vector potential $\boldsymbol{A}=M \boldsymbol{\omega}_{\perp} \times \boldsymbol{r}$; the resulting uniform magnetic field is $\boldsymbol{B}=2 M \boldsymbol{\omega}_{\perp}$. Unfortunately, the usual LLL picture treats electrons with negative charge, and the corresponding one-body states involve reinterpretations of some of the quantum numbers, as discussed in pp. 750757 of Cohen-Tannoudii et al. (1977). For this reason, I choose to work directly from the original Hamiltonian $H_{0}^{\prime}=H_{0}-\boldsymbol{\Omega} \cdot \boldsymbol{L}$, because the role of $n_{+}$and $n_{-}$is particularly transparent.

Another interesting feature of the transformation to a quadratic form in $\boldsymbol{p}-q \boldsymbol{A}$ is the possibility of topological gauge potentials. In the context of optical lattices, Jaksch and Zoller (2003) propose inducing various terms in the single-particle Hamiltonian that mimic the effect of 
an external magnetic field. Specifically, when a two-level atom hops around a closed path in the lattice, external lasers can generate a net phase change that is essentially equivalent to the magnetic flux of an applied field [see, (Mueller, 2004; Satija et al., 2006; Sørensen et al., 2005) for alternative approaches, including the possibility of "non-Abelian" gauge potentials].

Similar ideas also apply to a simple trapped condensate. Juzeliūnas et al. (2005) propose using a three-level system in the " $\Lambda$ " configuration [two nearly degenerate lower levels, each coupled to a higher third state by external laser beams (Arimondo, 1996); for a "dressed-atom approach" to these three-level systems, see, for example, pp. 451-454 of Cohen-Tannoudii et al. (1998)]. Such an interacting three-level system has a "dark" state that effectively uncouples from the remaining states. If one of the external lasers has orbital angular momentum [see, for example, Sec. 52 of Gottfried (1966) and Allen et al. (2003, 1999)], then the dark state experiences a nontrivial topological gauge potential that in principle can act like an essentially arbitrary magnetic field (and hence an arbitrary applied vorticity for the trapped condensate). Whether such methods can indeed generate angular momentum in the condensate and the associated vortices remains uncertain. More work (both theory and experiment) is clearly desirable.

\section{LLL condensate wave functions}

Ho (2001) proposes a linear combination of the LLL single-particle states in Eq. (5.11) as the GP condensate wave function for the rapidly rotating two-dimensional BEC

$$
\psi_{L L L}(\boldsymbol{r})=\sum_{m \geq 0} c_{m} \psi_{m}(\boldsymbol{r})=f(\zeta) e^{-r^{2} /\left(2 d_{\perp}^{2}\right)}
$$

where $f(\zeta)=\sum_{m \geq 0} c_{m} \zeta^{m}$ is an analytic function of the complex variable $\zeta$ [compare Sec. III.C; see also pp. 587592 of Shankar (1994)]. Specifically, for a truncated basis set, the analytic function $f(\zeta)$ is a complex polynomial and thus has a factorized form

$$
f(\zeta) \propto \prod_{j}\left(\zeta-\zeta_{j}\right)
$$

apart from an overall constant factor. Evidently, $f(\zeta)$ vanishes at each of the points $\zeta_{j}$, which are the positions of the nodes of the condensate wave function $\psi_{L L L}(\boldsymbol{r})$. In addition, the phase of this wave function increases by $2 \pi$ whenever $\zeta$ moves in the positive sense around any of these zeros. Thus the points $\zeta_{j}$ are precisely the positions of the vortices in the trial state $\psi_{L L L}$, and minimization with respect to the constants $c_{m}$ is effectively the same as minimization with respect to the position of the vortices (Butts and Rokhsar, 1999).

\section{Unrestricted minimization}

This trial state has some very interesting and unusual properties. Apart from the overall Gaussian envelope, the spacing of the vortices completely determines the spatial variation of the number density $n_{L L L}(\boldsymbol{r})=$ $\left|\psi_{L L L}(\boldsymbol{r})\right|^{2}=|f(\zeta)|^{2} e^{-r^{2} / d_{\perp}^{2}}$. The number density varies smoothly between adjacent vortices because $|f(\zeta)|^{2}$ consists of harmonic functions. Hence the vortex core size is comparable with $l=\sqrt{\hbar /(M \Omega)}$, which is essentially $d_{\perp}$ in the rapidly rotating limit. In contrast to the ThomasFermi limit at lower angular velocity, the present trial function $\psi_{L L L}$ automatically incorporates all the kinetic energy.

It is important to emphasize that the one-body state $\psi_{L L L}$ arises from the GP energy functional and that the corresponding many-body state

$$
\Psi_{G P}\left(\boldsymbol{r}_{1}, \boldsymbol{r}_{2}, \cdots, \boldsymbol{r}_{N}\right) \propto \prod_{n=1}^{N} \psi_{L L L}\left(\boldsymbol{r}_{n}\right)
$$

is just a Hartree product of these LLL states. For any such ground state, the system exhibits "off-diagonal" long-range order (Penrose and Onsager, 1956; Yang, 1962), and $\psi_{L L L}$ is the corresponding macroscopically occupied condensate wave function. Thus the mean-field LLL regime still has a BEC and, in general, exhibits superfluidity.

In the limit of rapid rotation, this LLL wave function can serve as a possible variational trial solution for the GP energy functional. Let $\langle\cdots\rangle_{L L L}$ denote an expectation value evaluated with $\psi_{L L L}$. Equations (5.7) and (5.12) show that $\left\langle L_{z}\right\rangle_{L L L} / \hbar=\left\langle a_{+}^{\dagger} a_{+}\right\rangle_{L L L}$ because $\left\langle a_{-}^{\dagger} a_{-}\right\rangle_{L L L}$ vanishes for any LLL state. Furthermore, the quantity $r^{2}=x^{2}+y^{2}$ is easily expressed as $(x+i y)(x-i y)=d_{\perp}^{2}\left(a_{-}+a_{+}^{\dagger}\right)\left(a_{+}+a_{-}^{\dagger}\right)$, whose expectation value gives $\left\langle r^{2}\right\rangle_{L L L} / d_{\perp}^{2}=\left\langle a_{+}^{\dagger} a_{+}\right\rangle_{L L L}+1$. Comparison yields the remarkably simple relation between the angular momentum and the mean-square radius Aftalion et al., 2005; Ho, 2001; Watanabe et al., 2004)

$$
\left\langle L_{z}\right\rangle_{L L L}=M \omega_{\perp}\left\langle r^{2}\right\rangle_{L L L}-\hbar
$$

for any linear combination of single-particle states in the lowest Landau level [as discussed in Sec. III.C, a slightly different form of this relation is important in the analysis of Butts and Rokhsar (1999)]. Note that this relation also follows directly from Eq. (5.13).

A combination of Eqs. (5.2) and (5.19) expresses the full GP energy functional (5.1) in the very intuitive and familiar form

$$
\begin{gathered}
E^{\prime}\left[\psi_{L L L}\right]=\hbar \Omega+\int d^{2} r\left[M \omega_{\perp}^{2}(1-\bar{\Omega}) r^{2}\left|\psi_{L L L}\right|^{2}\right. \\
\left.+\frac{1}{2} g_{2 D}\left|\psi_{L L L}\right|^{4}\right]
\end{gathered}
$$

where the integrand again involves only $r^{2}\left|\psi_{L L L}\right|^{2}$ and $\left|\psi_{L L L}\right|^{4}$ [compare Eq. (2.24)]. Unrestricted minimization 
with respect to $\left|\psi_{L L L}\right|^{2}$ at fixed normalization readily yields the number density

$$
\left|\psi_{\min }(r)\right|^{2}=n_{\min }(r)=n_{\min }(0)\left(1-\frac{r^{2}}{R_{0}^{2}}\right),
$$

where the (two-dimensional) central density is $n_{\min }(0)=$ $\mu_{\min } / g_{2 D}$, and the condensate radius is given by

$$
R_{0}^{2}=\frac{\mu_{\min }}{M \omega_{\perp}^{2}(1-\bar{\Omega})}
$$

Remarkably, this expression has the same parabolic form as the usual TF result in Eq. 2.27), even though the present functional explicitly includes all the kinetic energy. It does, however, rely on the restriction to the lowest Landau level.

The normalization condition $\int d^{2} r\left|\psi_{\min }\right|^{2}=N$ yields $N=\frac{1}{2} \pi R_{0}^{2} n_{\min }(0)$, which is equivalent to

$$
R_{0}^{2}=\frac{2 N g_{2 D}}{\pi \mu_{\min }}
$$

The product of Eqs. (5.22) and (5.23) gives an explicit expression for $R_{0}^{4}$

$$
R_{0}^{4}=\frac{2 N g_{2 D}}{\pi M \omega_{\perp}^{2}(1-\bar{\Omega})}=\frac{8 N a d_{\perp}^{4}}{Z(1-\bar{\Omega})}
$$

that explicitly shows the radial expansion for $\bar{\Omega} \rightarrow 1$. Similarly, the ratio of the same two equations yields the chemical potential

$$
\mu_{\min }=\sqrt{\frac{2 N g_{2 D} M \omega_{\perp}^{2}(1-\bar{\Omega})}{\pi}}=\sqrt{\frac{8 N a(1-\bar{\Omega})}{Z}} \hbar \omega_{\perp} .
$$

The first form is similar to Eq. (4.9) in the mean-field TF regime (the different fractional powers arise from the different dimensions for the normalization integrals), whereas the second form gives the condition $\mu_{\text {min }} \lesssim 2 \hbar \omega_{\perp}$ for the validity of the mean-field LLL regime

$$
1-\bar{\Omega} \lesssim \frac{Z}{2 N a}
$$

If $Z / 2 a \sim 100$ and $N \sim 10^{4}$, then a dimensionless rotation speed $\bar{\Omega} \sim 0.99$ just barely approaches the mean-field LLL regime.

The energy of this approximate minimizing mean-field LLL density $\left|\psi_{\text {min }}\right|^{2}$ follows immediately by evaluating Eq. (5.20)

$$
\frac{E_{\min }^{\prime}-\hbar \Omega}{N \hbar \omega_{\perp}}=\frac{2 \mu_{\min }}{3 \hbar \omega_{\perp}}=\frac{4}{3} \sqrt{\frac{2 N a(1-\bar{\Omega})}{Z}} .
$$

As emphasized by Aftalion et al. (2005), the approximate density in Eq. (5.21) arises from an unrestricted variation and is not within the LLL. Thus the actual energy will be higher, because the minimization then includes the vortices through the zeros of the analytic function in
Eq. (5.17). As seen below, the main effect of the vortices is to increase the numerical coefficient in Eq. (5.27), keeping the same dependence on the various parameters.

The derivative of Eq. (5.27) with respect to $\Omega$ yields the angular momentum of the minimizing mean-field LLL state

$$
\left(L_{z}\right)_{\min }=\frac{1}{3} M N R_{0}^{2} \omega_{\perp}-\hbar,
$$

which also follows from Eq. (5.19). Here, the first term is just the solid-body value (since direct integration with the parabolic $\left|\psi_{\min }\right|^{2}$ yields $\left.\left\langle r^{2}\right\rangle_{\min }=\frac{1}{3} N R_{0}^{2}\right)$. Unlike the mean-field TF regime discussed in Sec. IV.A, the LLL reduction from the classical value is only of order $N^{-1}$ and thus negligible in the thermodynamic limit.

\section{Inclusion of the vortices}

The minimizing parabolic density profile in Eq. (5.21) cannot provide a full description of the rotating LLL condensate, for it omits the fine-grained structure associated with the vortices.

a. renormalization of $g_{2 D}$ In the present mean-field LLL regime, the intervortex separation $\sim d_{\perp}$ is small compared to the size of the condensate. Thus it is possible to write the number density obtained from Eq. (5.16) in the form $\left|\psi_{L L L}(\boldsymbol{r})\right|^{2} \approx\left|\bar{\psi}_{L L L}(\boldsymbol{r})\right|^{2}|m(\boldsymbol{r})|^{2}$ as the product of a slowly varying envelope function $\left|\bar{\psi}_{L L L}(\boldsymbol{r})\right|^{2}$ and a rapidly varying modulation function $|m(\boldsymbol{r})|^{2}$ that vanishes at the center of each vortex (Aftalion et al., 2005; Bavm and Pethick, 2004; Fetter, 2001; Fischer and Bavm, 2003; Watanabe et al., 2004). For a large vortex lattice, this modulating function is essentially periodic, and it is convenient to normalize it so that $\int_{\text {cell }} d^{2} r|m(\boldsymbol{r})|^{2}=1$ over each unit cell. Substitution of this approximate density into the LLL energy functional (5.20) yields products of slowly varying functions involving powers of $\left|\bar{\psi}_{L L L}(\boldsymbol{r})\right|^{2}$ and rapidly varying periodic modulating functions involving powers of $|m(\boldsymbol{r})|^{2}$.

The integral over the condensate separates into a sum of integrals over each unit cell, with the slowly varying quantities acting as locally constant factors. Thus the only effect of the vortex lattice is that the interaction term acquires a numerical factor $\beta=\int_{\text {cell }} d^{2} r|m(\boldsymbol{r})|^{4}$, which effectively renormalizes the interaction constant $g_{2 D} \rightarrow \beta g_{2 D}$ Aftalion et al. (2005) discuss these steps carefully]. For an unbounded triangular vortex lattice, the resulting $\beta \approx 1.1596$ is the numerical value for the corresponding Abrikosov vortex lattice (Abrikosov, 1957; Kleiner et al., 1964). Consequently, the radius $R_{0}$ in Eq. (5.24) expands by the factor $\beta^{1 / 4} \approx 1.0377$.

b. nonuniformity of the vortex array To study the effect of the vortices in more detail, $\mathrm{Ho}$ (2001) considers the 
logarithm of the particle density for a LLL condensate wave function of the form in Eq. (5.16), obtaining

$$
\ln \left|\psi_{L L L}(\boldsymbol{r})\right|^{2}=\ln n_{L L L}(\boldsymbol{r})=-\frac{r^{2}}{d_{\perp}^{2}}+2 \sum_{j} \ln \left|\boldsymbol{r}-\boldsymbol{r}_{j}\right|,
$$

where $\boldsymbol{r}_{j}$ is the position of the $j$ th vortex. The twodimensional Laplacian of this equation yields

$$
\nabla^{2} \ln n_{L L L}(\boldsymbol{r})=-\frac{4}{d_{\perp}^{2}}+4 \pi \sum_{j} \delta^{(2)}\left(\boldsymbol{r}-\boldsymbol{r}_{j}\right)
$$

because $\nabla^{2} \ln \left|\boldsymbol{r}-\boldsymbol{r}_{j}\right|=2 \pi \delta^{(2)}\left(\boldsymbol{r}-\boldsymbol{r}_{j}\right)$. The sum over the delta functions is precisely the two-dimensional vortex density $n_{v}(\boldsymbol{r})$, which gives a striking relation between the particle density $n_{L L L}(\boldsymbol{r})$ in the mean-field LLL regime and the vortex density (Aftalion et al., 2005; $\mathrm{Ho}$, 2001; Watanabe et al., 2004)

$$
n_{v}(\boldsymbol{r})=\frac{1}{\pi d_{\perp}^{2}}+\frac{1}{4 \pi} \nabla^{2} \ln n_{L L L}(\boldsymbol{r}) .
$$

The first term is an effective vortex density $n_{\text {eff }}=$ $M \omega_{\perp} /(\pi \hbar)$ for $\Omega=\omega_{\perp}$, and the second is a (small) correction.

To appreciate the implications of this elegant equation, first assume that the vortex density is uniform with the actual Feynman value $\pi n_{v}=m \Omega / \hbar$. Equation (5.31) then has the Gaussian solution $n_{L L L}(r)=$ $n_{L L L}(0) \exp \left(-r^{2} / \sigma^{2}\right)$, where $\sigma^{-2}=\pi\left(n_{\text {eff }}-n_{v}\right)$; equivalently, the effective squared condensate radius is

$$
\sigma^{2}=\frac{\hbar}{M\left(\omega_{\perp}-\Omega\right)}=\frac{d_{\perp}^{2}}{1-\bar{\Omega}} .
$$

As expected from the radial expansion, the length $\sigma$ diverges as $\Omega \rightarrow \omega_{\perp}$.

In fact, Cooper et al. (2004) use numerical methods to show that a small distortion of the vortex lattice can both lower the energy and significantly change the mean density profile from Gaussian to one closely resembling the parabolic shape in Eq. (5.21). Thus it is better to substitute this profile into Eq. (5.31) to find the approximate and slightly nonuniform vortex density Aftalion et al., 2005; Watanabe et al., 2004)

$$
n_{v}(r) \approx \frac{1}{\pi d_{\perp}^{2}}-\frac{1}{\pi R_{0}^{2}} \frac{1}{\left(1-r^{2} / R_{0}^{2}\right)^{2}} .
$$

This expression is similar to the result in the mean-field Thomas-Fermi limit in Eq. (4.24), and the correction is again small, of order $1 / \mathcal{N}_{v}$.

\section{Rapidly rotating anisotropic traps}

In practice, most traps are not exactly axisymmetric (namely, $\omega_{x} \neq \omega_{y}$, and I take $\omega_{x}<\omega_{y}$ for definiteness). The rotational properties of such realistic traps differ in certain significant ways from those of symmetric traps. Similar problems arise in the study of deformed nuclei (Valatin, 1956). If the anistropic trap is stationary, the noninteracting single-particle ground state is simply a product of two one-dimensional Gaussians in $x$ and $y$ with oscillator lengths $d_{x}$ and $d_{y}$. With increasing rotation speed $\Omega$, however, the coupling term $-\Omega L_{z}$ mixes the $x$ and $y$ components. As a result, the ground-state density elongates along the direction of weak confinement (here along $x$ ) and diverges as $\Omega \rightarrow \omega_{x}$ (Linn et al. 2001).

In complete analogy with the operators $a_{ \pm}$and $a_{ \pm}^{\dagger}$ for the symmetric trap [see Eq. (5.5)], a rotating anisotropic trap has a similar set of creation and destruction operators, but their form is rather more intricate (Fetter, 2007; Valatin, 1956). Nevertheless, in the limit $\Omega \rightarrow \omega_{x}$, the lowest single-particle states constitute a nearly degenerate set that again acts like the lowest Landau level. The corresponding LLL condensate wave function again involves a polynomial in a scaled complex variable (a linear combination of $x$ and $i y$ ). Consequently, many of the results derived for the mean-field LLL regime in an isotropic trap continue to apply, at least qualitatively, for the rapidly rotating anisotropic trap.

One important difference is the significant anisotropy of the condensate as $\Omega \rightarrow \omega_{x}$ (Fetter, 2007; Linn et al., 2001). Indeed, Sánchez-Lotero and Palacios (2005) and Sinha and Shlyapnikov (2005) both model this situation with a one-dimensional effectively infinite strip of finite width, containing one or more rows of vortices. As discussed in Sec. VI below, however, this behavior may well be unobservable. Before reaching such a one-dimensional limit, there may well be a quantum phase transition to a correlated many-body state (sometimes called a "vortex liquid") that is not superfluid and has no BEC. Such questions currently remain unanswered and merit more study.

\section{QUANTUM PHASE TRANSITION TO HIGHLY CORRELATED STATES}

The mean-field LLL regime relies on the assumption that the interaction energy per particle $\sim \mu$ is small compared to the gap $2 \hbar \omega_{\perp}$, leading to the lower bound for $\bar{\Omega}$ [compare Eq. (5.26)]

$$
1-\frac{Z}{2 N \tilde{a}} \lesssim \bar{\Omega}
$$

where $\tilde{a}=\beta a$ is the renormalized scattering length that includes the vortex-induced density variation (note that $\beta \approx 1.1596$ is simply a numerical factor, see Sec. V.C.2 for more details). In practice, typical experiments require $\bar{\Omega} \gtrsim 0.99$ to reach this regime. For the present purpose, it is important to emphasize that the mean-field LLL regime has a BEC with macroscopic occupation and is indeed a superfluid. Specifically, the associated manybody GP ground state is simply the Hartree product in Eq. (5.18). 
For still larger values of $\bar{\Omega}$, however, the mean-field LLL regime should eventually disappear through a quantum phase transition, leading to a wholly different highly correlated many-body ground state $\Psi_{\text {corr. }}$. Unlike the mean-field LLL ground state, the new ground state $\Psi_{\text {corr }}$ does not have a BEC and is not a superfluid. As evidence for such a transition, the exact two-dimensional ground state for bosons with small $N$ and relatively large fixed angular momentum $L_{z}$ indeed has a correlated form (Wilkin and Gunn, 2000; Wilkin et al., 1998). Furthermore, detailed numerical studies (Cooper et al., 2001) explicitly exhibit such a phase transition.

To understand the physics of this quantum phase transition, it is helpful to focus on the number of vortices $\mathcal{N}_{v}$. In the mean-field LLL regime, the two-dimensional condensate has a radius $R_{0}$, and Eq. (4.6) yields the number of vortices $\mathcal{N}_{v}=R_{0}^{2} / l^{2} \approx R_{0}^{2} / d_{\perp}^{2}$. Use of Eq. (5.24) and the discussion in Sec. V.C.2 give

$$
\mathcal{N}_{v} \approx \frac{R_{0}^{2}}{d_{\perp}^{2}}=\sqrt{\frac{8 N \tilde{a}}{Z(1-\bar{\Omega})}}
$$

As an alternative derivation of this result (Bloch et al., 2007), note that the $m$ th LLL state $\psi_{m}$ has a meansquare radius $\sim m d_{\perp}^{2}$ for $m \gg 1$ [Eq. [5.13)]. If the sum in Eq. (5.16) extends to $m_{\max }$, then the condensate has an effective squared radius $\left\langle r^{2}\right\rangle_{m_{\max }} \approx m_{\max } d_{\perp}^{2}$. The number of vortices (the degree of the polynomial) is $\mathcal{N}_{v}=m_{\max }$, which is the same as Eq. (6.2) found directly from the Feynman relation for the vortex density. The parameters used below Eq. (5.26) yield $\mathcal{N}_{v} \approx 200$.

It is valuable to consider the ratio $\nu \equiv$ $N / \mathcal{N}_{v}$ (Cooper et al., 2001; Wilkin and Gunn, 2000) known as the "filling factor" or "filling fraction" because of a similar quantity in the fractional quantum Hall effect [see, for example, Chap. 1 of Prange and Girvin (1987)]. In the mean-field LLL regime, it has the simple form

$$
\nu \equiv \frac{N}{\mathcal{N}_{v}}=\sqrt{\frac{Z(1-\bar{\Omega}) N}{8 \tilde{a}}},
$$

but it also remains well defined for general manybody ground states of rotating bosons [and for charged fermions in a magnetic field, which is its original role (Prange and Girvin, 1987)].

In the mean-field LLL approach for weakly interacting bosons in a rotating trap, typified by the work of Butts and Rokhsar (1999) and discussed in Secs. III.C and V.C, the equilibrium state is a vortex array that breaks rotational symmetry and is not an eigenstate of $L_{z}$. As an alternative, Cooper et al. (2001) use exact diagonalization to study the ground states for $\mathcal{N}_{v}=4,6$, and 8 and for many values of $N$ [see also (Cooper and Wilkin, 1999; Viefers et al., 2000; Wilkin and Gunn, 2000) for less extensive studies]. This numerical analysis uses a toroidal geometry with periodic boundary conditions in both directions, allowing a study of bosonic systems with relatively large integral values of both $N$ and $\mathcal{N}_{v}$. For a particular aspect ratio of the torus that can accommodate a triangular array with $\mathcal{N}_{v}=8$, they investigate filling fractions $\nu$ ranging through several rational values from $\frac{1}{2}$ up to 9 . They also study the mean-field GP ground state containing a vortex lattice with $\mathcal{N}_{v}=8$ for the same values of $\nu$. Comparison of these two classes of states shows that the GP vortex lattice is the ground state for $\nu_{c} \gtrsim 6$, where the specific numerical value depends on the aspect ratio of the torus (and hence the number of vortices that can fit into the periodic structure). Other choices of this aspect ratio yield critical values in the range $\nu_{c} \sim 6-10$.

A combination of Eq. (6.1) for the validity of the mean-field LLL regime and the lower bound $\nu \gtrsim 1$ for the mean-field GP ground state yields the inequalities that define the allowed range of the mean-field LLL state (Bloch et al., 2007)

$$
1-\frac{Z}{2 N \tilde{a}} \lesssim \bar{\Omega} \lesssim 1-\frac{8 \tilde{a}}{Z N}
$$

The right-hand inequality for $\bar{\Omega}$ illustrates the experimental difficulty of studying the quantum phase transition. Since $Z / a$ typically exceeds 100 , Eq. (6.3) makes it clear that reaching even $\nu \sim 10$ will require significant reduction of $N$ (note that the high rotation speed $\bar{\Omega} \sim 0.999$ presents a daunting experimental challenge).

In contrast to the vortex lattices for $\nu>\nu_{c}$, the ground states for smaller $\nu<\nu_{c}$ are rotationally symmetric incompressible vortex liquids that are eigenstates of $L_{z}$. They have close similarities to the bosonic analogs of the Jain (1989) sequence of fractional quantum Hall states that describe a two-dimensional electron gas in a strong magnetic field. The simplest of these many-body ground states is the bosonic Laughlin state (Laughlin, 1983)

$$
\begin{aligned}
& \Psi_{\text {Laughlin }}\left(\boldsymbol{r}_{1}, \boldsymbol{r}_{2}, \cdots, \boldsymbol{r}_{N}\right) \\
& \propto \prod_{n<n^{\prime}}^{N}\left(z_{n}-z_{n^{\prime}}\right)^{2} \exp \left(-\frac{1}{2} \sum_{n=1}^{N}\left|z_{n}\right|^{2}\right),
\end{aligned}
$$

where $z_{n}=\left(x_{n}+i y_{n}\right) / d_{\perp}$ refers to the $n$th particle. Here the power 2 ensures that this state is symmetric under the interchange of any two particles (in contrast to the fermionic case with power 3). Cooper and Wilkin (1999); Wilkin and Gunn (2000); Wilkin et al. (1998) note that this state occurs for $\nu=\frac{1}{2}$.

It is worth emphasizing the difference between this Laughlin ground state and the GP Hartree ground state in Eq. (5.18). For the Laughlin state in Eq. (6.5), the first (product) factor means that there is no offdiagonal long-range order (Penrose and Onsager, 1956; Yang, 1962) and hence no BEC. In addition, the Laughlin state vanishes whenever two particles come together, enforcing the many-body correlations. Indeed, the usual short-range two-body potential $V\left(\boldsymbol{r}-\boldsymbol{r}^{\prime}\right)=$ $g \delta\left(\boldsymbol{r}-\boldsymbol{r}^{\prime}\right)$ has zero expectation value in this correlated state (Trugman and Kivelson, 1985). In contrast, the GP ground state has every particle in the same single-particle 
Hartree state $\psi_{L L L}$; thus particles in the GP condensate tend to overlap wherever $\left|\psi_{L L L}(\boldsymbol{r})\right|$ is large.

Sinova et al. (2002) provide an appealing physical picture of the onset of this quantum phase transition. Using various approximate descriptions, they find that the long-wavelength spectrum of collective modes becomes quadratic in the limit of rapid rotations, in contrast to the familiar linear Bogoliubov spectrum in Eq. (2.44). This softened spectrum implies that the vortex lattice melts for a critical filling fraction $\nu_{c} \approx 8$, in reasonable agreement with the estimates of Cooper et al. (2001). An alternative description of the vortex-lattice melting (Baym, 2005) relies on the softening of the Tkachenko vortexlattice modes, as seen in Eq. (4.28). The connection between these apparently distinct pictures remains uncertain.

The periodic toroidal geometry of Cooper et al. (2001) has the strong advantage of avoiding boundary effects; Regnault and Jolicoueur (2003, 2004) use a spherical geometry to perform a similar numerical analysis. Both groups propose specific sequences of known quantum Hall states for the rotating dilute Bose gas when $\frac{1}{2}<\nu<\nu_{c}$, although there is currently no experimental evidence for such sequences. Nevertheless, Cooper et al. (2001) find strong overlap between their results from exact diagonalization and the Laughlin state $\left(\nu=\frac{1}{2}\right)$ and other similar but more complicated quantum Hall states for the particular filling fractions $\nu=1, \frac{3}{2}, 2, \frac{5}{2}, 6$. For a more detailed discussion of these various quantum Hall states, see, for example, (Cooper et al., 2001; Regnault and Jolicoueur, 2003, 2004) and Sec. VII.C of Bloch et al. (2007).

What is the physics of this ground-state transition from a vortex lattice to a vortex liquid? Why is the critical value of the filling fraction $\nu_{c} \sim 5-10$, as opposed to $\nu_{c} \sim 1$ or $\nu_{c} \sim 0.1$ ? To think about this question, it is helpful to consider $N$ bosonic particles in a plane, with $2 N$ degrees of freedom. Vortices appear as the system rotates, and the corresponding vortex coordinates provide $\mathcal{N}_{v}$ collective degrees of freedom. ${ }^{12}$ This relation between particle and collective degrees of freedom is familiar in many areas of many-body theory: For example, the Debye theory of specific heat uses the phonon normal modes instead of the particle positions in the crystal, and the original number of particle degrees of freedom $(3 N)$ also determines the cutoff frequency for the phonon spectrum [see, for example, Chap. 23 of Ashcroft and Mermin $(1976)]$. Similarly, the theory of a charged electron gas often relies on collective density modes (plasma oscillations) instead of the particle coordinates (Pines, 1961).

For a slowly rotating Bose-Einstein gas in a plane, the $2 N$ particle coordinates provide a convenient description. In principle, the $\mathcal{N}_{v}$ collective vortex degrees of freedom

12 Note that vortices obey first-order dynamical equations, so that the associated $x$ and $y$ coordinates are not independent; in particular, they can serve as a pair of Hamiltonian canonical variables (Fetter, 1967; Haldane and Wu, 1985). should reduce the original total $2 N$ degrees of freedom to $2 N-\mathcal{N}_{v}$, but the effect is unimportant as long as $\mathcal{N}_{v} \ll N$. When $\mathcal{N}_{v}$ becomes comparable with $N$, however, the depletion of the particle degrees of freedom becomes crucial, ultimately producing a phase transition to a wholly different ground state. With this picture, the critical value $\nu_{c} \sim 5-10$ is not surprising, since $\mathcal{N}_{v} / N=1 / \nu$ is still relatively small.

If experiments can indeed create these correlated states, how might they be detected? Section VII.C of Bloch et al. (2007) proposes several techniques, including (1) a reduced three-body recombination rate [because of the anti-correlation between the bosons inherent in the first factor in Eq. (6.5)] and (2) the characteristic density profile of these various quantum Hall states. Another possibility would be to study the ensemble averaged density-density noise correlations (Altman et al., 2004; Brown and Twiss, 1956), which should exhibit a different structure in the correlated state from that in the mean-field LLL regime.

\section{OUTLOOK}

As this review demonstrates in detail, experimental and theoretical studies of rotating Bose-Einstein condensates generally agree well for scaled angular velocities $\bar{\Omega} \equiv \Omega / \omega_{\perp}$ up to $\sim 0.995$.

\section{A. Successes}

These accomplishments include several different regimes.

1. For slow rotation $\bar{\Omega} \equiv \Omega / \omega_{\perp} \ll 1$ (Sec. III), the condensate includes only a few vortices, and centrifugal effects are negligible. Thus the overall density profile is essentially that of the nonrotating condensate, apart from the small holes associated with the vortex cores. Experimental observations of vortex precession in one- and two-component superfluids (see Sec. III.D.1) provide the only direct measurements of vortex dynamics in dilute BECs.

2. As the rotation increases, the centrifugal effects become important, leading to significant changes in the condensate's aspect ratio (Sec. IV). For not too large $\bar{\Omega}$, however, the intervortex spacing $\sim l=$ $[\hbar /(M \Omega)]^{1 / 2}$ remains large compared to the vortex core size $\xi$. In this mean-field Thomas-Fermi regime, the gradient energy associated with density variation remains negligible, and the hydrodynamic flow velocity $\boldsymbol{v}=\hbar \boldsymbol{\nabla} S / M$ predominates in the energy balance. Typically this case holds for $0.75 \lesssim \bar{\Omega} \lesssim 0.99$.

3. Finally, for very large rotation speed $0.99 \lesssim \bar{\Omega} \lesssim$ 0.999, the vortex cores expand, filling much of the 
available space. The energy associated with spatial variation of the density now becomes comparable to the hydrodynamic flow energy. In this mean-field lowest-Landau-level regime (Sec. V), the single-particle part of the energy in the rotating frame is exactly soluble, leading to the rotational analog of the Landau levels for an electron in a uniform magnetic field. In particular, there is an energy gap $\sim 2 \hbar \omega_{\perp}$ between the nearly degenerate lowest Landau level and the first excited Landau level. In addition, the condensate expands radially, decreasing both the central particle density and the mean interaction energy $\mu$, which thus becomes smaller than the energy gap $2 \hbar \omega_{\perp}$. The set of lowest-Landau-level states then provides a convenient and flexible description of the equilibrium of the rapidly rotating condensate. Although current experiments with $\mu /\left(2 \hbar \omega_{\perp}\right) \approx 0.6$ only just reach this mean-field LLL regime (see Sec. V.A), it is reasonably well understood.

\section{B. Challenges}

Despite the many remarkable achievements of the past decade, several theoretical predictions of considerable interest remain unverified experimentally.

\section{Rapid rotation of both Bose and Fermi gases in the quantum Hall regime at small filling fraction}

For dilute ultracold trapped single-component Bose gases of the sort considered in this review, one obvious challenge for future experiments is the quantum phase transition from a superfluid with a Bose-Einstein condensate to a nonsuperfluid correlated state. As discussed in Sec. VI, formation of this new state requires large angular momentum and smaller particle number $N$ than is typical in most current experiments. Whether such states can indeed be realized is uncertain. In principle, one might start with an anharmonic trap of the sort considered in Sec. IV.C. Once the condensate rotates with $\bar{\Omega} \gtrsim 1$, it might be feasible adiabatically to turn off the external laser beam (and hence the anharmonic component of the trap potential), raising the effective $\omega_{\perp}$ and leaving the system with high angular velocity $\bar{\Omega} \lesssim 1$.

Another possibility is be to rely on topological gauge potentials (Sec. V.B), in which applied laser fields with orbital angular momentum can produce effective nonuniform topological "magnetic" fields that are more general than the usual constant $\boldsymbol{B}$. To my knowledge, all studies of rapidly rotating trapped bosons rely on the detailed form of the operator $-\boldsymbol{\Omega} \cdot \boldsymbol{L}=-\boldsymbol{A} \cdot \boldsymbol{p}$, with $\boldsymbol{A}=\boldsymbol{\Omega} \times \boldsymbol{r}$ as the equivalent vector potential. The existence of a nonsuperfluid correlated many-body state reflects the high degeneracy of the lowest Landau level, which in turn depends on the specific form of the effective vector potential ( $\boldsymbol{A}=\boldsymbol{\Omega} \times \boldsymbol{r}$, apart from a possible gauge transfor- mation). It is not obvious that such nonsuperfluid correlated many-body states occur for more general topological gauge potentials. This question certainly merits additional detailed study.

A related area for future activity is the study of rapidly rotating Fermi gases. At present, this theoretical topic is only partially explored [see, for example, (Bhongale et al., 2007; Möller and Cooper, 2007; Zhai and Ho, 2006)], and much work remains. The only relevant experiments are those of Zwierlein et al. (2005) on the vortex lattice in rotating fermionic ${ }^{6} \mathrm{Li}$ (see Sec. IV.B.6). The experiment starts with a BEC of tightly bound bosonic molecules and then sweeps across the Feshbach resonance toward the more weakly bound fermionic regime. Unfortunately, any move far to the BCS side of the transition at fixed low temperature necessarily encounters the severely reduced critical temperature of the weak-coupling BCS limit.

As a pure theoretical argument, let us consider the weak-coupling fermionic ground state as a function of $\bar{\Omega}=\Omega / \omega_{\perp}$. For intermediate $\bar{\Omega}$, a vortex lattice forms, as in Zwierlein et al. (2005). What happens for larger $\bar{\Omega}$ ? Is there a transition to a normal state similar to that at $H_{c 2}$ in a type-II superconductor [see, for example, Secs. 4.7, 4.8, and 5.3.3 of Tinkham (1996)]? Or does a transition to a correlated quantum Hall state intervene? If so, for reasonable experimental parameters, how does the filling fraction $\nu$ relate to $\bar{\Omega}$ ? These intriguing questions deserve careful study.

\section{Rotation of ultracold dilute gases with long-range dipole-dipole interactions}

Dilute quantum gases with long-range anisotropic dipole-dipole interactions constitute a fascinating generalization of the usual case with short-range isotropic interactions characterized by a scattering length $a$. Although the general dipole-dipole potential is rather complicated, experiments usually use a strong uniform field to orient the dipoles (either magnetic or electric) along the $\hat{z}$ direction. Such fields lead to the simpler interaction potential [see, for example, the review by Menotti and Lewenstein (2007) and the experimental studies of ${ }^{52} \mathrm{Cr}$ (Griesmaier et al., 2005; Koch et al., 2007; Lahave et al., 2007)]

$$
V_{\mathrm{dd}}\left(\boldsymbol{r}-\boldsymbol{r}^{\prime}\right)=\frac{d^{2}}{\left|\boldsymbol{r}-\boldsymbol{r}^{\prime}\right|^{3}}\left[1-3(\hat{\boldsymbol{z}} \cdot \hat{\boldsymbol{n}})^{2}\right],
$$

where $d$ is the relevant dipole moment and $\hat{\boldsymbol{n}}$ is a unit vector along the direction $\boldsymbol{r}-\boldsymbol{r}^{\prime}$ joining the two atoms.

The dipole-dipole potential in Eq. (7.1) is repulsive if $\hat{\boldsymbol{z}} \cdot \hat{\boldsymbol{n}}$ vanishes (namely if the oriented dipoles lie in the $x y$ plane), whereas it is attractive if $(\hat{\boldsymbol{z}} \cdot \hat{\boldsymbol{n}})^{2}>\frac{1}{3}$ (in particular, for oriented dipoles located along the $\hat{z}$ axis). Since a BEC with attractive interactions tends to collapse, a long cigar-shaped condensate with dipole-dipole interactions should be unstable, but a flat disk-shaped conden- 
sate should be stable. Koch et al. (2007) use a Feshbach resonance to decrease the $s$-wave scattering length, thus enhancing the relative role of $V_{\mathrm{dd}}$. These experiments confirm the qualitative expectations in great detail.

Recent theoretical studies have predicted many properties of the ground states of rotating BECs with dipolar interactions. In the weak-interaction limit, Cooper et al. (2005) [see also (Zhang and Zhai, 2005)] propose that an increased dipolar coupling strength induces a series of transitions in the mean-field (large filling fraction $\nu$ ) condensate involving vortex lattices of varying symmetry (triangular, square, stripe, and "bubble" phases). They also study the behavior for small $\nu$, when the system is no longer superfluid. Subsequently, Komineas and Cooper (2007) analyze the more general situation as a function of both the dipolar interaction and the chemical potential relative to the $s$-wave contact interaction.

Another system of great interest is the rapidly rotating dipolar Fermi gas, whose predicted ground state (Baranov et al., 2005) has a simple incompressible form for filling fraction $\nu=\frac{1}{3}$. For smaller $\nu$, however, theory predicts that the quantum Hall states ultimately become unstable to the formation of crystalline Wigner states (Osterloh et al., 2007). Experimental studies of such nonsuperfluid fermionic states involve many technical issues that remain for future investigation.

\section{Rotating spinor condensates}

The development of optical laser traps that confine all the magnetic substates (Stamper-Kurn et al., 1998) allows a direct study of spin-one BECs (as well as higherspin cases). These experiments use a focused (reddetuned) infrared laser that attracts the BEC to the waist of the beam. For a general theoretical introduction, see Sec. III.E, along with Sec. 12.2 of Pethick and Smith (2002), Sec. 3.4 of Fetter (2002), and the original papers Ho (1998) and Ohmi and Machida (1998). In contrast to the two-component mixtures of the different hyperfine states $F=1$ and $F=2$ (see Secs. III.D.1 and IV.B.5), the rotational invariance of the interaction between two atoms with the same $F=1$ now imposes special restrictions on the allowed interaction potential. The macroscopic order parameter becomes a spin-one object with three components

$$
\boldsymbol{\Psi}=\left(\begin{array}{c}
\Psi_{1} \\
\Psi_{0} \\
\Psi_{-1}
\end{array}\right)
$$

labeled by the $m_{F}$ value. In the low-energy limit where only $s$-wave scattering is relevant, the effective shortrange interaction has the form

$$
V_{\mathrm{int}}\left(\boldsymbol{r}_{1}-\boldsymbol{r}_{2}\right)=\left(g_{0}+g_{2} \boldsymbol{F}_{1} \cdot \boldsymbol{F}_{2}\right) \delta^{(3)}\left(\boldsymbol{r}_{1}-\boldsymbol{r}_{2}\right),
$$

where $\boldsymbol{F}_{j}$ is the hyperfine spin of the $j$ th atom. The coupling constant $g_{0}$ is generally positive, but $g_{2}$ is proportional to $a_{2}-a_{0}$ (the difference of the scattering lengths in the two symmetric channels $\boldsymbol{F}=\boldsymbol{F}_{1}+\boldsymbol{F}_{2}=0$ or 2). Thus $g_{2}$ can be either positive or negative, depending on the details of the two-body scattering.

A mean-field description ( $\mathrm{Ho}, 1998)$ uses an effective energy functional and writes the spinor order parameter in a factored form $\Psi_{\alpha}(\boldsymbol{r})=\sqrt{n(\boldsymbol{r})} \zeta_{\alpha}(\boldsymbol{r})$. Here, $\alpha$ runs over the three allowed values $\alpha=(1,0,-1), n(\boldsymbol{r})$ is the assumed common density for all three spin components, and $\zeta_{\alpha}(\boldsymbol{r})$ is a normalized spinor with $\boldsymbol{\zeta}^{\dagger} \cdot \boldsymbol{\zeta}=1$. Apart from the gradient term in the effective energy, the ground-state spinor follows by minimizing the spindependent part of the energy density $\frac{1}{2} n^{2} g_{2}\langle\boldsymbol{F}\rangle^{2}$, where $\langle\boldsymbol{F}\rangle=\sum_{\alpha \beta} \zeta_{\alpha}^{*} \boldsymbol{F}_{\alpha \beta} \zeta_{\beta}$.

If $g_{2}$ is positive, then the minimum energy occurs for $|\langle\boldsymbol{F}\rangle|=0$. Such states are known as "polar" or "antiferromagnetic," and they follow by spatial rotations of the hyperfine state $\left|m_{F}=0\right\rangle$ [this situation applies for ${ }^{23} \mathrm{Na}$ (Stenger et al., 1998)]. If, however, $g_{2}$ is negative, then the minimum energy is for a "ferromagnetic" state with $|\langle\boldsymbol{F}\rangle|=1$, obtained by spatial rotations of the state $\left|m_{F}=1\right\rangle$ [this situation applies for ${ }^{87} \mathrm{Rb}$ (Sadler et al. , 2006; Vengalattore et al., 2007)].

The multicomponent structure of the spinor order parameter in Eq. (7.2) is reminiscent of the order parameter for superfluid ${ }^{3} \mathrm{He}$, where each atom has a nuclear spin $1 / 2$ and zero net electronic spin. Low-temperature experiments show that ${ }^{3} \mathrm{He}$ forms a $p$-wave paired superfluid, with the two atoms in a triplet nuclear spin state and unit relative orbital angular momentum [see, for example, Vollhardt and Wölfle (1990)]. In particular, the ${ }^{3} \mathrm{He}$ order parameter has several components, analogous to those for a spinor BEC. Many groups have studied the structure of vortices in rotating ${ }^{3} \mathrm{He}$, with various proposed candidates, both for a single vortex and for vortex lattices [for detailed reviews, see Fetter (1986); Salomaa and Volovik (1987)].

Unfortunately, the semi-phenomenological GinzburgLandau theory for superfluid ${ }^{3} \mathrm{He}$ is often inadequate to predict the detailed experimental phase diagram for various vortices as a function of pressure and temperature. Specifically, the energy difference between many of the vortex states is small, and the relevant phenomenological Ginzburg-Landau parameters have considerable uncertainties their experimental values [see, for example, Secs. 7.5 and 7.6 of Vollhardt and Wölfle (1990)]. Consequently, experimental studies provide essential guidance for theoretical analyses. Eltsov et al. (2005) and Finne et al. (2006) review recent experimental work on vortices and other related structures in superfuid ${ }^{3} \mathrm{He}$.

In an unbounded dilute trapped BEC with a singlecomponent order parameter, the vortex core size and structure arise from the balance of the kinetic energy and the interaction energy (see Sec. III.A). For a trapped BEC with a single component, the new feature is the additional energy of the confining trap, and various regimes occur depending on the dimensionless interaction parameter $\mathrm{Na} / d_{0}$ (see Secs. II.B and III.B.1). If $N a / d_{0} \lesssim 1$, then the vortex core is comparable with the condensate 
radius (both of order $d_{0}$, the mean oscillator length) or the intervortex separation (see Sec. III.C). In the more typical Thomas-Fermi regime with $N a / d_{0} \gg 1$, however, the vortex core radius $\xi$ (the healing length) is much smaller than both $d_{0}$ and the still larger mean condensate radius $R_{0}$.

For a three-component spinor condensate, the spatial variation of each component provides additional freedom in minimizing the overall free energy. Since the resulting coupled GP field equations are nonlinear, several solutions can exist, at least in principle, each corresponding to a local minimum of the GP energy [see Sec. 7.5.7 of Vollhardt and Wölfle (1990) for the similar situation involving the Ginzburg-Landau theory of superfluid ${ }^{3} \mathrm{He}$. In practice, it turns out that $\left|g_{2}\right| \ll g_{0}$ for both ${ }^{23} \mathrm{Na}$ and ${ }^{87} \mathrm{Rb}$ (Yip, 1999), which means that gradient energies and other interaction energies play a significant role in determining the condensate wave function. This near degeneracy is especially important in understanding the core of a quantized vortex in a spinor condensate, because these various energies compete with the energy of the circulating flow. Yip (1999) proposes several different vortex structures, and many other authors have amplified these considerations, both for single vortices [see, for example, (Bulgakov and Sadreev, 2003; Isoshima et al., 2001; Mizushima et al., 2002) and references therein] and for vortex lattices [see, for example, (Mizushima et al., 2004) and references therein]. One experimental challenge in studying a rotating spinor BEC is ensuring both the full axisymmetry of the optical trap and its alignment with the axis of rotation; otherwise the lifetime decreases significantly. These difficulties appear in the experiments on a rotating paired Fermi gas, where the magnetic field associated with a Feshbach resonance also requires an optical trap [see Sec. IV.B.6 and Zwierlein et al. (2005)].

\section{Acknowledgments}

I thank A. Aftalion, E. Demler, M. Krusius, D. Roberts, N. Wilkin, and E. Zaremba for valuable discussions and suggestions. Part of this work was completed during a stay at the Institut Henri Poincaré - Centre Emile Borel, Paris, and at the Kavli Institute for Theoretical Physics, Santa Barbara, where the research was supported in part by the National Science Foundation under Grant No. PHY05-51164. I thank these institutions for their hospitality and support.

\section{References}

Abers, E. S., 2004, Quantum Mechanics (Pearson Prentice Hall, Upper Saddle River, New Jersey).

Abo-Shaeer, J. R., C. Raman, J. M. Vogels, and W. Ketterle, 2001, Science 292, 476.

Abrikosov, A. A., 1957, Zh. Eksp. Teor. Fiz. [Sov. Phys.-JETP 5, $1174(1957)] \mathbf{3 2}, 1442$.
Aftalion, A., 2006, Vortices in Bose-Einstein Condensates (Birkhäuser Boston).

Aftalion, A., X. Blanc, and J. Dalibard, 2005, Phys. Rev. A 71, 023611.

Aftalion, A., and I. Danaila, 2004, Phys. Rev. A 69, 033608.

Aftalion, A., and T. Rivière, 2001, Phys. Rev. A 64, 043611.

Allen, L., S. M. Barnett, and M. J. Padgett (eds.), 2003, Optical Angular Momentum (Institute of Physics Publishing, Bristol).

Allen, L., and J. H. Eberly, 1987, Optical Resonance and TwoLevel Atoms (Dover Publications, New York).

Allen, L., M. J. Padgett, and M. Babikar, 1999, Prog. Opt. 39, 291.

Altman, E., E. Demler, and M. D. Lukin, 2004, Phys. Rev. A 70, 013603.

Andersen, M. F., C. Ryu, P. Cladé, V. Natarajan, A. Vaziri, K. Helmerson, and W. D. Phillips, 2006, Phys. Rev. Lett. 97, 170406.

Anderson, B. P., P. C. Haljan, C. A. Regal, D. L. Feder, L. A. Collins, C. W. Clark, and E. A. Cornell, 2001, Phys. Rev. Lett. 86, 2926.

Anderson, B. P., P. C. Haljan, C. E. Wieman, and E. A. Cornell, 2000, Phys. Rev. Lett. 85, 2857.

Anderson, B. P., and M. A. Kasevich, 1998, Science 282, 1686.

Anderson, M. H., J. R. Ensher, M. H. Matthews, C. E. Wieman, and E. A. Cornell, 1995, Science 269, 198.

Andrews, M. R., C. G. Townsend, H.-J. Miesner, D. S. Durfee, D. M. Kurn, and W. Ketterle, 1997, Science 275, 637.

Anglin, J. R., 2002, Phys. Rev. A 65, 063611.

Anglin, J. R., and M. Crescimanno, 2002, Inhomogeneous vortex matter, cond-mat/0210063.

Arimondo, E., 1996, Prog. Opt. 35, 257.

Ashcroft, N. W., and N. D. Mermin, 1976, Solid State Physics (Holt, Rinehart and Winston).

Baksmaty, L. O., S. J. Woo, S. Choi, and N. P. Bigelow, 2004, Phys. Rev. Lett. 92, 160405.

Baranov, M. H., K. Osterloh, and M. Lewenstein, 2005, Phys. Rev. Lett. 94, 070404.

Baym, G., 2003, Phys. Rev. Lett. 91, 110402.

Baym, G., 2005, J. Low Temp. Phys. 138, 601.

Baym, G., and C. J. Pethick, 1996, Phys. Rev. Lett. 76, 6.

Baym, G., and C. J. Pethick, 2004, Phys. Rev. A 69, 043619.

Berezinskii, V. L., 1971, Zh. Eksp. Teor. Fiz. [Sov. Phys.JETP 34, 610 (1972)] 61, 1144.

Berry, M. V., 1984, Proc. Roy. Soc. London A 392, 45.

Bhongale, S. G., M. R. Goosen, and S. J. J. M. F. Kokkelmans, 2007, BCS-BEC crossover in a strongly correlated Fermi gas, arXiv:0710:5288.

Bishop, D. J., and J. D. Reppy, 1978, Phys. Rev. Lett. 40, 1727.

Bloch, I., J. Dalibard, and W. Zwerger, 2007, Many-body physics with ultracold gases, arXiv:0704.3011v2, to be published in Rev. Mod. Phys.

Bogoliubov, N. N., 1947, J. Phys. (USSR) 11, 23.

Bradley, C. C., C. A. Sackett, and R. G. Hulet, 1997, Phys. Rev. Lett. 78, 985.

Bradley, C. C., C. A. Sackett, J. J. Tollett, and R. G. Hulet, 1995, Phys. Rev. Lett. 75, 1687.

Bretin, V., P. Rosenbusch, F. Chevy, G. V. Shlyapnikov, and J. Dalibard, 2003, Phys. Rev. Lett. 90, 100403.

Bretin, V., S. Stock, Y. Seurin, and J. Dalibard, 2004, Phys. Rev. Lett. 92, 050403.

Brown, L. M. (ed.), 2000, Selected Papers of Richard Feynman 
(World Scientific, Singapore).

Brown, R. H., and R. Q. Twiss, 1956, Nature (London) 177, 27.

Bulgakov, E. N., and A. F. Sadreev, 2003, Phys. Rev. Lett. 90, 200401.

Butts, D. A., and D. S. Rokhsar, 1999, Nature (London) 397, 327.

Campbell, L. J., and R. M. Ziff, 1979, Phys. Rev. B 20, 1886.

Castin, Y., 2006, Basic theory tools for degenerate Fermi gases, arXiv:cond-mat/0612613v2.

Castin, Y., and R. Dum, 1999, Eur. Phys. J. D 7, 399.

Chandrasekharan, K., 1985, Elliptic Functions (SpringerVerlag, Berlin).

Chevy, F., K. W. Madison, and J. Dalibard, 2000, Phys. Rev. Lett. 85, 2223.

Coddington, I., P. Engels, V. Schweikhard, and E. A. Cornell, 2003, Phys. Rev. Lett. 91, 100402.

Coddington, I., P. C. Haljan, P. Engels, V. Schweikhard, S. Tung, and E. A. Cornell, 2004, Phys. Rev. A 70, 063607.

Cohen-Tannoudji, C., B. Diu, and F. Lalöe, 1977, Quantum Mechanics (Wiley, New York).

Cohen-Tannoudji, C., J. Dupont-Roc, and G. Grynberg, 1998, Atom-Photon Interactions: Basic Processes and Applications (Wiley, New York).

Colson, W. B., and A. L. Fetter, 1978, J. Low Temp. Phys. 33, 231 .

Cooper, N. R., S. Komineas, and N. Read, 2004, Phys. Rev. A 70, 033604.

Cooper, N. R., E. H. Rezayi, and S. H. Simon, 2005, Phys. Rev. Lett. 95, 200402.

Cooper, N. R., and N. K. Wilkin, 1999, Phys. Rev. B 60, $16279(\mathrm{R}$.

Cooper, N. R., N. K. Wilkin, and J. M. F. Gunn, 2001, Phys. Rev. Lett. 87, 120405.

Cornell, E. A., D. S. Hall, M. R. Matthews, and C. E. Weiman, 1998, J. Low Temp. Phys. 113, 151.

Cozzini, M., and S. Stringari, 2003, Phys. Rev. A 67, 041602(R).

Cozzini, M., S. Stringari, and C. Tozzo, 2006, Phys. Rev. A 73, 023615.

Dalfovo, F., S. Giorgini, L. Pitaevskii, and S. Stringari, 1999, Rev. Mod. Phys. 71, 463.

Dalfovo, F., and S. Stringari, 1996, Phys. Rev. A 53, 2477.

Dalibard, J., 2007, private communication.

Davis, K. B., M.-O. Mewes, M. R. Andrews, N. J. van Druten, D. S. Durfee, D. M. Kurn, and W. Ketterle, 1995, Phys. Rev. Lett. 75, 3969

DeConde, K., and R. E. Packard, 1975, Phys. Rev. Lett. 35, 732.

Dodd, R. J., K. Burnett, M. Edwards, and C. W. Clark, 1997, Phys. Rev. A 56, 587.

Donnelly, R. J., 1991, Quantized Vortices in Helium II (Cambridge University Press, Cambridge).

Dutton, Z., M. Budde, C. Slowe, and L. V. Hau, 2001, Science 293, 663 .

Edwards, M., P. A. Ruprecht, K. Burnett, R. J. Dodd, and C. W. Clark, 1996, Phys. Rev. Lett. 77, 1671.

Eltsov, V. B., M. Krusius, and G. E. Volovik, 2005, Prog. Low Temp. Phys. XV, 1.

Engels, P., I. Coddington, P. C. Haljan, and E. A. Cornell, 2002, Phys. Rev. Lett. 89, 100403.

Engels, P., I. Coddington, P. C. Haljan, V. Schweikhard, and E. A. Cornell, 2003, Phys. Rev. Lett. 90, 170405.

Esry, B. D., C. H. Greene, J. P. Burke, and J. L. Bohn, 1997,
Phys. Rev. Lett. 78, 3594

Feder, D. L., and C. W. Clark, 2001, Phys. Rev. Lett. 87, 190401.

Feder, D. L., M. S. Pindzola, L. A. Collins, B. I. Schneider, and C. W. Clark, 2000, Phys. Rev. A 62, 053606.

Feder, D. L., A. A. Svidzinsky, A. L. Fetter, and C. W. Clark, 2001, Phys. Rev. Lett. 86, 564.

Fedichev, P. O., A. E. Muryshev, and G. V. Shlyapnikov, 1999, Phys. Rev. A 60, 3220.

Fetter, A. L., 1967, Phys. Rev. 162, 143.

Fetter, A. L., 1969, in Lectures in Theoretical Physics, edited by K. T. Mahanthappa and W. E. Britten (Gordon and Breach, N. Y., 1969), volume XIB, p. 351.

Fetter, A. L., 1972, Ann. Phys. (N.Y.) 70, 67.

Fetter, A. L., 1974, J. Low Temp. Phys. 16, 533.

Fetter, A. L., 1986, Prog. Low Temp. Phys. X, 1.

Fetter, A. L., 1996, Phys. Rev. A 53, 4245.

Fetter, A. L., 1998, J. Low Temp. Phys. 113, 198.

Fetter, A. L., 2001, Phys. Rev. A 64, 063608.

Fetter, A. L., 2002, J. Low Temp. Phys. 129, 263.

Fetter, A. L., 2007, Phys. Rev. A 75, 013620.

Fetter, A. L., and K. Harvey, 1971, Phys. Rev. A 4, 2305.

Fetter, A. L., B. Jackson, and S. Stringari, 2005, Phys. Rev. A 71, 013605.

Fetter, A. L., and A. A. Svidzinsky, 2001, J. Phys.: Condens. Matter 13, R135.

Fetter, A. L., and J. D. Walecka, 2003, Quantum Theory of Many-Particle Systems (Dover Publications, Inc.).

Fetter, A. L., and J. D. Walecka, 2006, Nonlinear Mechanics: A Supplement to Theoretical Mechanics of Particles and Continua (Dover Publications, Inc.).

Feynman, R. P., 1955, Prog. Low Temp. Phys. I, 17.

Feynman, R. P., 1965, The Feynman Lectures on Physics: Quantum Mechanics, volume III (Addison-Wesley, Reading, MA).

Finne, A. P., V. B. Eltsov, R. Hänninen, N. B. Kopnin, J. Kopu, M. Krusius, M. Tsubota, and G. E. Volovik, 2006, Rep. Prog. Phys. 69, 3157.

Fischer, U. R., and G. Baym, 2003, Phys. Rev. Lett. 90, 140402.

Fu, H., and E. Zaremba, 2006, Phys. Rev. A 73, 013614

García-Ripoll, J. J., and V. M. Pérez-García, 2001a, Phys. Rev. A 63, 041603.

García-Ripoll, J. J., and V. M. Pérez-García, 2001b, Phys. Rev. A 64, 053611.

de Gennes, P. G., 1966, Superconductivity of Metals and Alloys (Benjamin, New York).

Ginzburg, V. L., and L. P. Pitaevskii, 1958, Zh. Eksp. Teor. Fiz. [Sov. Phys.-JETP 7, 858 (1958)] 34, 1240.

Giorgini, S., L. P. Pitaevskii, and S. Stringari, 2007, Theory of ultracold Fermi gases, arXiv:0706.3360v1.

Gottfried, K., 1966, Quantum Mechanics, volume I (Benjamin, New York and Amsterdam).

Greiner, M., O. Mandel, T. Esslinger, T. W. Hänsch, and I. Bloch, 2002, Nature (London) 415, 39.

Griesmaier, A., J. Werner, S. Hensler, J. Stuhler, and T. Pfau, 2005, Phys. Rev. Lett. 94, 160401.

Griffiths, D. J., 2005, Introduction to Quantum Mechanics (Pearson Prentice Hall, Upper Saddle River, New Jersey), second edition.

Gross, E. P., 1961, Nuovo Cimento 20, 454.

Hadzibabic, Z., P. Krüger, M. Cheneau, B. Battelier, and J. Dalibard, 2006, Nature (London) 441, 1118.

Haldane, F. D., and Y.-S. Wu, 1985, Phys. Rev. Lett. 55, 
2887.

Haljan, P. C., B. P. Anderson, I. Coddington, and E. A. Cornell, 2001a, Phys. Rev. Lett. 86, 2922.

Haljan, P. C., I. Coddington, P. Engels, and E. A. Cornell, 2001b, Phys. Rev. Lett. 87, 210403.

Hall, D. S., M. R. Matthews, C. E. Wieman, and E. A. Cornell, 1998, Phys. Rev. Lett. 81, 1543.

Ho, T.-L., 1998, Phys. Rev. Lett. 81, 742.

Ho, T.-L., 2001, Phys. Rev. Lett. 87, 060403.

Hodby, E., G. Hechenblaikner, S. A. Hopkins, O. M. Maragò, and C. J. Foot, 2002, Phys. Rev. Lett. 88, 010405.

Hohenberg, P. C., 1967, Phys. Rev. 158, 383.

Hohenberg, P. C., and P. C. Martin, 1965, Ann. Phys. (N.Y.) 34, 291 .

Inguscio, M., S. Stringari, and C. E. Weiman (eds.), 1999, Bose-Einstein Condensation in Atomic Gases (IOP Press, Amsterdam).

Isoshima, T., K. Machida, and T. Ohmi, 2001, J. Phys. Soc. Jpn. 70, 1604.

Isoshima, T., M. Nakahara, T. Ohmi, and K. Machida, 2000, Phys. Rev. A 61, 063610.

Jain, J. K., 1989, Phys. Rev. Lett. 63, 199.

Jaksch, D., C. Bruder, J. I. Cirac, C. W. Gardiner, and P. Zoller, 1998, Phys. Rev. Lett. 81, 3108.

Jaksch, D., and P. Zoller, 2003, New J. Phys. 5, 56.1.

Josephson, B. D., 1962, Phys. Lett. 1, 251.

Juzeliūnas, G., P. Öhberg, J. Ruseckas, and A. Klein, 2005, Phys. Rev. A 71, 053614.

Kasamatsu, K., and M. Tsubota, 2007, Quantized vortices in atomic Bose-Einstein condensates, arXiv:0709.1042v2.

Kasamatsu, K., M. Tsubota, and M. Ueda, 2002, Phys. Rev. A 66, 053606.

Kasamatsu, K., M. Tsubota, and M. Ueda, 2003, Phys. Rev. Lett. 91, 150406.

Kavoulakis, G. M., and G. Baym, 2003, New J. Phys. 5, 51.1.

Khawaja, U. A., 2005, Phys. Rev. A 71, 063611.

Kim, J.-K., and A. L. Fetter, 2005, Phys. Rev. A 72, 023619.

Kleiner, W. H., L. M. Roth, and S. H. Autler, 1964, Phys. Rev. 133, A1226.

Koch, T., T. Lahaye, J. Metz, B. Fröhlich, A. Griesmaier, and T. Pfau, 2007, Stabilizing a purely dipolar quantum gas against collapse, arXiv:0710:3643.

Köhler, T., K. Góral, and P. S. Julienne, 2006, Rev. Mod. Phys. 78, 1311.

Komineas, S., and N. R. Cooper, 2007, Phys. Rev. A 75, 023623.

Kosterlitz, J. M., and D. J. Thouless, 1973, J. Phys. C 6, 1181.

Krüger, P., 2007, private communication.

Lahaye, T., T. Koch, B. Fröhlich, M. Fattori, J. Metz, A. Griesmaier, S. Giovanazzi, and T. Pfau, 2007, Nature (London) 448, 672.

Lamb, H., 1945, Hydrodynamics (Dover, New York), 6th edition.

Landau, L. D., 1941, J. Phys. (USSR) 5, 71.

Landau, L. D., and E. M. Lifshitz, 1960, Mechanics (Pergamon Press, Oxford).

Landau, L. D., and E. M. Lifshitz, 1965, Quantum Mechanics Non-relativistic Theory (Pergamon Press, Oxford), second edition.

Landau, L. D., and E. M. Lifshitz, 1987, Fluid Mechanics (Pergamon Press, Oxford), second edition.

Laughlin, R. B., 1983, Phys. Rev. Lett. 50, 1395.

Leanhardt, A. E., A. Görlitz, A. P. Chikkatur, D. Kielpinski,
Y., D. E. Pritchard, and W. Ketterle, 2002, Phys. Rev. Lett. 89, 190403.

Leggett, A. J., 2001, Rev. Mod. Phys. 73, 307.

Leggett, A. J., 2006, Quantum Liquids: Bose condensation and Cooper pairing in condensed-matter systems (Oxford University Press).

Lifshitz, E. M., and L. P. Pitaevskii, 1980a, Statistical Physics, volume 1 (Pergamon Press, Oxford), third edition.

Lifshitz, E. M., and L. P. Pitaevskii, 1980b, Statistical Physics, volume 2 (Pergamon Press, Oxford), third edition. Linn, M., and A. L. Fetter, 1999, Phys. Rev. A 60, 4910.

Linn, M., M. Niemeyer, and A. L. Fetter, 2001, Phys. Rev. A 64, 023602.

London, F., 1954, Superfluids: Microscopic Theory of Superfluid Helium, volume II (Dover Publications, New York).

Lundh, E., 2002, Phys. Rev. A 65, 043604.

Lundh, E., and P. Ao, 2000, Phys. Rev. A 61, 063612.

Lundh, E., C. J. Pethick, and H. Smith, 1997, Phys. Rev. A 55, 2126.

Madison, K. W., F. Chevy, F. Bretin, and J. Dalibard, 2001, Phys. Rev. Lett. 86, 4443.

Madison, K. W., F. Chevy, W. Wohlleben, and J. Dalibard, 2000a, Phys. Rev. Lett. 84, 806.

Madison, K. W., F. Chevy, W. Wohlleben, and J. Dalibard, 2000b, J. Mod. Opt. 47, 2715.

Martikainen, J.-P., and H. T. C. Stoof, 2003, Phys. Rev. Lett. 91, 240403.

Mason, P., N. G. Berloff, and A. L. Fetter, 2006, Phys. Rev. A 74, 043611 .

Matthews, M. R., B. P. Anderson, P. C. Haljan, D. S. Hall, M. J. Holland, J. E. Williams, C. E. Wieman, and E. A. Cornell, 1999a, Phys. Rev. Lett. 83, 3358.

Matthews, M. R., B. P. Anderson, P. C. Haljan, D. S. Hall, C. E. Wieman, and E. A. Cornell, 1999b, Phys. Rev. Lett. 83, 2498 .

McGee, S. A., and M. J. Holland, 2001, Phys. Rev. A 63, 043608.

Menotti, C., and M. Lewenstein, 2007, Ultra-cold dipolar gases, arXiv:0711:3406.

Mermin, N. D., and H. Wagner, 1966, Phys. Rev. Lett. 17, 1133.

Messiah, A., 1961, Quantum Mechanics, volume I (Wiley, New York).

Mizushima, T., M. Ichioka, and K. Machida, 2003, Phys. Rev. Lett. 90, 180401.

Mizushima, T., N. Kobayashi, and K. Machida, 2004, Phys. Rev. A 70, 043613.

Mizushima, T., K. Machida, and T. Kita, 2002, Phys. Rev. Lett. 89, 030401.

Modugno, M., L. Pricoupenko, and Y. Castin, 2003, Eur. Phys. J. D 22, 235.

Moerdijk, A. J., B. J. Verhaar, and A. Axelsson, 1995, Phys. Rev. A 51, 4852.

Möller, G., and N. R. Cooper, 2007, Phys. Rev. Lett. 99, 190409

Möttönen, M., T. Mizushima, T. Isoshima, M. M. Salomaa, and K. Machida, 2003, Phys. Rev. A 68, 023611.

Mueller, E. J., 2004, Phys. Rev. A 70, 041603(R).

Mueller, E. J., and T.-L. Ho, 2002, Phys. Rev. Lett. 88, 180403.

Mueller, E. J., and T.-L. Ho, 2003, Phys. Rev. A 67, 063602.

Muryshev, A. E., H. B. van Linden van den Heuvell, and G. V. Shlyapnikov, 1999, Phys. Rev. A 60, R2665.

Myatt, C. J., E. A. Burt, R. W. Ghrist, E. A. Cornell, and 
C. E. Wieman, 1997, Phys. Rev. Lett. 78, 586.

Nepomnyashchi, Y. A., 1974, Teor. Mat. Phys. 20, 399.

Nozières, P., and D. Saint James, 1982, J. Phys. (Paris) 43, 1133.

Ogawa, S.-I., M. Möttönen, M. Nakahara, T. Ohmi, and H. Shimada, 2002, Phys. Rev. A 66, 013617.

Ohmi, T., and K. Machida, 1998, J. Phys. Soc. Jpn. 67, 1822.

Onsager, L., 1949, Nuovo Cimento Suppl. 6, 249.

Osterloh, K., N. Barberán, and M. Lewenstein, 2007, Phys. Rev. Lett. 99, 160403.

Packard, R. E., and T. M. Sanders, 1972, Phys. Rev. A 6, 799.

Parker, N. G., B. Jackson, A. M. Martin, and C. S. Adams, 2007, Vortices in Bose-Einstein condensates: theory, arXiv:0704.0146v1.

Pathria, R. K., 1996, Statistical Mechanics (ButterworthHeineman, Oxford), second edition.

Penrose, O., and L. Onsager, 1956, Phys. Rev. 104, 576.

Pérez-García, V. M., H. Michinel, J. I. Cirac, M. Lewenstein, and P. Zoller, 1996, Phys. Rev. Lett. 76, 5320.

Pethick, C. J., and H. Smith, 2002, Bose-Einstein Condensation in Dilute Gases (Cambridge University Press).

Pines, D., 1961, The Many-Body Problem (Benjamin, New York).

Pismen, L. M., 1999, Vortices in Nonlinear Fields (Clarendon Press, Oxford).

Pismen, L. M., and B. Y. Rubinstein, 1991, Physica D 47, 353.

Pitaevskii, L., and S. Stringari, 2003, Bose-Einstein Condensation (Clarendon Press, Oxford).

Pitaevskii, L. P., 1961, Zh. Eksp. Teor. Fiz. [Sov. Phys.-JETP 13, 451 (1961)] 40, 646.

Pollock, F., 1967, Phys. Fluids 10, 473.

Prange, R. E., and S. M. Girvin (eds.), 1987, The Quantum Hall Effect (Springer-Verlag, New York).

Pu, H., C. K. Law, J. H. Eberly, and N. P. Bigelow, 1999, Phys. Rev. A 59, 1533.

Raman, C., J. R. Abo-Shaeer, J. M. Vogels, K. Xu, and W. Ketterle, 2001, Phys. Rev. Lett. 87, 210402.

Regnault, N., and T. Jolicoueur, 2003, Phys. Rev. Lett. 91, 030402.

Regnault, N., and T. Jolicoueur, 2004, Phys. Rev. A 69, 235309.

Resnick, D. J., J. C. Garland, J. T. Boyd, S. Shoemaker, and R. S. Newrock, 1981, Phys. Rev. Lett. 47, 1542.

Rokhsar, D. S., 1997a, Dilute bose gas in a torus: vortices and persistent currents, cond-mat/9709212.

Rokhsar, D. S., 1997b, Phys. Rev. Lett. 79, 2164.

Rosenbusch, P., V. Bretin, and J. Dalibard, 2002, Phys. Rev. Lett. 89, 200403.

Rubinstein, B. Y., and L. M. Pismen, 1994, Physics D 78, 1.

Ruprecht, P. A., M. J. Holland, K. Burnett, and M. Edwards, 1995, Phys. Rev. A 51, 4704.

Ryu, C., M. F. Andersen, P. Cladé, V. Natarajan, K. Helmerson, and W. D. Phillips, 2007, Plys. Rev. Lett. 99, 260401.

Sadler, L. E., J. M. Higbie, S. R. Leslie, M. Vengalattore, and D. M. Stamper-Kurn, 2006, Nature (London) 443, 312.

Salomaa, M. M., and G. E. Volovik, 1987, Rev. Mod. Phys. $\mathbf{5 9}, 533$.

Sánchez-Lotero, P., and J. J. Palacios, 2005, Phys. Rev. A 72, 043613.

Satija, I. I., D. C. Dakin, and C. W. Clark, 2006, Phys. Rev. Lett. 97, 216401.

Scherer, D. R., C. N. Weiler, T. N. Neely, and B. P. Anderson,
2007, Phys. Rev. Lett. 98, 110402.

Schiff, L. I., 1968, Quantum Mechanics (McGraw-Hill, New York), third edition.

Schweikhard, V., I. Coddington, P. Engels, V. P. Mogendorff, and E. A. Cornell, 2004, Phys. Rev. Lett. 92, 040404.

Schweikhard, V., S. Tung, and E. A. Cornell, 2007, Phys. Rev. Lett 99, 030401.

Sensarma, R., M. Randeria, and T.-L. Ho, 2006, Phys. Rev. Lett. 96, 090403.

Shankar, R., 1994, Principles of Quantum Mechanics (Plenum Press, New York and London), second edition.

Sheehy, D. E., and L. Radzihovsky, 2004a, Phys. Rev. A 70, 051602(R).

Sheehy, D. E., and L. Radzihovsky, 2004b, Phys. Rev. A 70, 063620.

Shin, Y., M. Saba, M. Vengalattore, T. A. Pasquini, C. Sanner, A. E. Leanhardt, M. Prentiss, D. E. Pritchard, and W. Ketterle, 2004, Phys. Rev. Lett. 93, 160406.

Simula, T. P., and P. B. Blakie, 2006, Phys. Rev. Lett. 96, 020404.

Sinha, S., 1997, Phys. Rev. A 55, 4325.

Sinha, S., and G. V. Shlyapnikov, 2005, Phys. Rev. Lett. 94, 150401.

Sinova, J., C. B. Hanna, and A. H. MacDonald, 2002, Phys. Rev. Lett. 89, 030403.

Sonin, E. B., 1987, Rev. Mod. Phys. 59, 87.

Sonin, E. B., 2005, Phys. Rev. A 71, 011603(R).

Sørensen, A., E. Demler, and M. Lukin, 2005, Phys. Rev. Lett. 94, 086803.

Stamper-Kurn, D. M., M. R. Andrews, A. P. Chikkatur, S. Inouye, H.-J. Miesner, J. Stenger, and W. Ketterle, 1998, Phys. Rev. Lett. 80, 2027.

Stenger, J., S. Inouye, D. M. Stamper-Kurn, H. J. Miesner, A. P. Chikkatur, and W. Ketterle, 1998, Nature (London) 396, 345 .

Stock, S., B. Battelier, V. Bretin, Z. Hadzibabic, and J. Dalibard, 2005a, Laser Phys. Lett. 2, 275.

Stock, S., V. Bretin, F. Chevy, and J. Dalibard, 2004, Europhys. Lett. 65, 594 .

Stock, S., Z. Hadzibzbic, B. Battelier, M. Cheneau, and J. Dalibard, 2005b, Phys. Rev. Lett. 95, 190403.

Stringari, S., 1996, Phys. Rev. Lett. 77, 2360.

Svidzinsky, A. A., and A. L. Fetter, 1998, Phys. Rev. A 58, 3168.

Svidzinsky, A. A., and A. L. Fetter, 2000a, Phys. Rev. Lett. $\mathbf{8 4}, 5919$.

Svidzinsky, A. A., and A. L. Fetter, 2000b, Phys. Rev. A 62, 063617.

Thomson, W., 1880, Philos. Mag. 10, 155.

Tinkham, M., 1996, Introduction to Superconductivity (McGraw-Hill, New York).

Tkachenko, V. K., 1966a, Zh. Eksp. Teor. Fiz. [Sov. Phys.JETP 22, 1282 (1966)] 49, 1875.

Tkachenko, V. K., 1966b, Zh. Eksp. Teor. Fiz. [Sov. Phys.JETP 23, 1049 (1966)] 50, 1573.

Tkachenko, V. K., 1969, Zh. Eksp. Teor. Fiz. [Sov. Phys.JETP 29, 949 (1969)] 56, 1763.

Trugman, S. A., and S. Kivelson, 1985, Phys. Rev. B 31, 5280.

Valatin, J. G., 1956, Proc. Roy. Soc. London 238, 132.

Vengalattore, M., S. R. Leslie, J. Guzman, and D. M. Stamper-Kurn, 2007, Spontaneously modulated spin textures in a dipolar spinor Bose-Einstein condensate, arXiv:0712.4182v1. 
Viefers, S., T. H. Hansson, and S. M. Reimann, 2000, Phys. Rev. A 62, 053604.

Vollhardt, D., and P. Wölfle, 1990, The Superfluid Phases of Helium 3 (Taylor and Francis, London).

Watanabe, G., G. Baym, and C. J. Pethick, 2004, Phys. Rev. Lett. 93, 190401.

Wilkin, N. K., and J. M. F. Gunn, 2000, Phys. Rev. Lett. 84, 6.

Wilkin, N. K., J. M. F. Gunn, and R. A. Smith, 1998, Phys. Rev. Lett. 80, 2265.

Williams, J., R. Walser, J. Cooper, E. A. Cornell, and M. Holland, 2000, Phys. Rev. A 61, 033612.

Williams, J. E., and M. J. Holland, 1999, Nature (London) 401, 568 .

Wolfram, S., 2007, http://functions.wolfram.com/.
Wu, B., and Q. Niu, 2001, Phys. Rev. A 64, R061603.

Yang, C. N., 1962, Rev. Mod. Phys. 34, 694.

Yarmchuk, E. J., M. J. V. Gordon, and R. E. Packard, 1979, Phys. Rev. Lett. 43, 214.

Yarmchuk, E. J., and R. E. Packard, 1982, J. Low Temp. Phys. 46, 479.

Yip, S.-K., 1999, Phys. Rev. Lett. 83, 4677.

Zambelli, F., and S. Stringari, 1998, Phys. Rev. Lett. 81, 1754.

Zhai, H., and T.-L. Ho, 2006, Phys. Rev. Lett. 97, 180414.

Zhang, J., and H. Zhai, 2005, Phys. Rev. Lett. 95, 200403.

Zwierlein, M. W., 2007, private communication.

Zwierlein, M. W., J. R. Abo-Shaeer, A. Schirotzek, C. H. Schunk, and W. Ketterle, 2005, Nature (London) 435, 1047. 